\title{
Microfluidic Flow Driven by Electric Fields
}

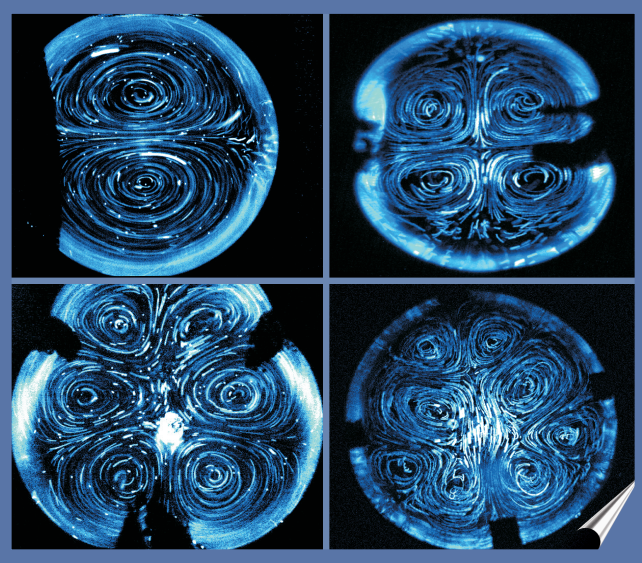

Dileep Mampallil 



\section{Microfluidic flow \\ driven by electric fields}

\section{DISSERTATION}

to obtain

the degree of doctor at the University of Twente, on the authority of the rector magnificus, prof. dr. H. Brinksma

on account of the decision of the graduation committee, to be publicly defended

on Thursday 22 September 2011 at $12.45 \mathrm{hrs}$

by

Dileep Mampallil Augustine

born on 20 May 1981

in Kanhirapuzha, India 
This dissertation has been approved by the promoter:

Prof. dr. F. Mugele

and the assistant-promoter:

Dr. H. T. M. van den Ende

Graduation committee:

Prof.dr. G. van der Steenhoven (chairman)

(University of Twente)

Prof.dr. R.G.H. Lammertink

(University of Twente)

Prof.dr. J.G.E. Gardeniers

(University of Twente)

Dr.ir. H.L. Offerhaus

(University of Twente)

Dr.ir. C.G.P.H. Schroen

(Wageningen University)

Prof.dr.ir. J.M.J. den Toonder

(Eindhoven University of Technology)

\section{MESA+}

The research study described in this thesis has been carried out in the group of Physics of Complex Fluids at University of Twente. The research is financially supported by MicroNed, the Microtechnology Research Programme of The Netherlands, under project II-B-2. Physics of Complex Fluids group is a part of the research program of the Institute for Mechanics, Processes and Control (IMPACT), MESA+ and the J.M. Burgerscentrum.

ISBN: 978-90-365-3252-5

DOI: $10.3990 . / 1.9789036532525$

Cover: Bottom view images of internal flows inside a drop (chapter 5).

Published by Dileep Mampallil

dileep.augustine@gmail.com

Copyright (c) 2011 by Dileep Mampallil

Cover design (C) 2011 by Dileep Mampallil

Printing: Gildeprint Drukkerijen, Enschede 
To Emilia, my parents and teachers 



\section{Contents}

1 Introduction 1

1.1 Microfluidics ................. 2

1.2 Electroosmosis . . . . . . . . . . . . 5

1.2.1 Electric double layer (EDL) . . . . . . . . 5

1.2.2 Potential in the EDL . . . . . . . . . . 6

1.2.3 Electroosmotic flow . . . . . . . . . 8

1.2.4 Electric modification of $\psi_{\zeta} \ldots \ldots \ldots$

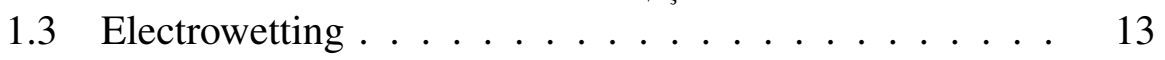

1.3.1 Surface tension . . . . . . . . . . . . 13

1.3.2 Effect of electric force at the contact line . . . . 14

1.3.3 Extensions of the classical electrowetting theory 17

Fine structure of the triple contact line . . . . . . 17

Electric double layer effects . . . . . . . . . . . 17

1.4 State of the art . . . . . . . . . . . . 18

1.5 Outline of the thesis . . . . . . . . . . 20

2 Experimental techniques 23

2.1 Device fabrication . . . . . . . . . . . . . . . . 24

2.1.1 Microchannels . . . . . . . . . . . 24

Soft lithography . . . . . . . . . . . . . . 24

Using capillaries . . . . . . . . . . . . 26

By Casting and cutting . . . . . . . . . . 26

2.1.2 Gate electrodes . . . . . . . . . . . . . 27

2.1.3 Insulating the gate electrodes . . . . . . . . 28 
2.1.4 Substrates for electrowetting . . . . . . . . 30

2.2 Imaging and analysis $\ldots \ldots \ldots . \ldots \ldots$

2.2.1 Image acquisition . . . . . . . . . . . . 33

2.2.2 Particle tracking . . . . . . . . . 34

3 Determining the surface charge of microfluidic channels 37

3.1 Introduction . . . . . . . . . . . . . 38

3.2 Modeling the channel current . . . . . . . . . . . . . . 40

3.3 Experiments .................... 44

3.3.1 Channel fabrication . . . . . . . . . . . 44

3.3.2 Current versus time measurements . . . . . . . 45

3.4 Results . . . . . . . . . . . . . . . 46

3.4 .1 Glass capillaries . . . . . . . . . . . 46

3.4.2 PDMS-glass channels . . . . . . . . . . . . . 48

3.5 Discussion . . . . . . . . . . . . . 51

3.6 Conclusion ................... 52

4 Electroosmotic shear flow in microchannels 53

4.1 Introduction . . . . . . . . . . . . . . . 54

4.2 Electroosmotic shear flow . . . . . . . . . . . . 55

4.2.1 Channel with gate electrodes . . . . . . . . . 59

4.2.2 Resistor network for the gate electrodes . . . . . 62

4.3 Experiments ...................... 62

4.3.1 Channel fabrication . . . . . . . . . . . 62

4.3.2 Measurements ............. 63

4.4 Results and discussion . . . . . . . . . . . . . 64

4.4.1 Chemically modified zeta potential . . . . . 64

4.4.2 Electrically modified zeta potential . . . . . . 66

4.5 Conclusion ................. 70

5 Controlling flow patterns in oscillating sessile drops 73

Part I: Controlling flow patterns . . . . . . . . . . . . . . . . . . 74

5.1 Introduction . . . . . . . . . . . . . . 74

5.2 Experiments . . . . . . . . . . . . 75

5.3 Results and discussion . . . . . . . . . . 76 
5.4 Conclusion . . . . . . . . . . . . . . 81

Part II: Sample pre-concentration . . . . . . . . . . . . . 82

5.5 Introduction . . . . . . . . . . . . . 82

5.6 Experiments . . . . . . . . . . . . . 83

5.7 Results and discussion . . . . . . . . . . . . 84

5.8 Conclusion . . . . . . . . . . . . . . . 91

6 Suppressing the coffee stain effect by electrowetting 93

6.1 Introduction . . . . . . . . . . . . . . . . . 94

6.2 Experimental methods . . . . . . . . . . . . 95

6.3 Results and discussion . . . . . . . . . . . . . 96

6.4 Conclusion . . . . . . . . . . . . . . 108

7 Oscillations of a sandwiched drop by electrowetting 109

7.1 Introduction . . . . . . . . . . . . . . . . . 110

7.2 Theory ......................... 111

7.2.1 The system description . . . . . . . . . . . . . 111

7.2.2 Equations of motion . . . . . . . . . . . 111

7.2.3 Calculating the pressure, $p \ldots \ldots \ldots 114$

7.2.4 The resonance frequencies . . . . . . . . . . . . 114

7.2.5 The profile of the drop . . . . . . . . . . . 116

7.2.6 The driven oscillations . . . . . . . . . . . 116

7.3 Experiments . . . . . . . . . . . . . . . 118

7.4 Results and discussion . . . . . . . . . . . . 119

7.5 Conclusion . . . . . . . . . . . . 125

8 Conclusion and outlook $\quad 127$

8.1 Conclusion . . . . . . . . . . . . . 128

8.2 Outlook ..................... 130

A Surface charge - pH -ionic concentration 133

B Calculation of the oscillation amplitude 137

C List of symbols $\quad 141$ 
Samenvatting

Bibliography

Acknowledgements

Publications 


\section{Chapter 1}

\section{Introduction}

The playground, where fluids and micro-technology meet, is called microfluidics. The manifold of possible applications in microfluidics has led to various studies on small scale fluid flows. The study described in this thesis has been inspired by two of the many interesting topics in microfluidics i.e. electroosmotic flow and electrowetting.

Electroosmotic flow is used to transport liquids through microfluidic channels. It exploits the two layers of charge, often called electric double layer, formed at the liquid-solid interface i.e. on the channel wall facing the liquid. By applying an electric field along the axis of the channel, a force can be applied on the mobile ions in this electric double layer, consequently creating a flow of the liquid. By modifying the strength of the electric double layer, the direction and magnitude of the flow in the channel can be controlled. For this purpose, an electric potential is applied to so called, gate electrodes, embedded in the channel walls. By creating different flow velocities near opposing walls of the channel, the liquid is sheared and the viscous properties can be explored.

Electrowetting, as the name says, is the modification of the wetting properties of a surface using electric forces. Electrowetting can be used to produce or transport discrete drops in microchannels or even between two solid substrates. These diminutive drops can act as reservoirs for chemical reactions or cell culture in biology etc. Such applications may 
benefit from mixing or stirring within these miniature drops.

This thesis describes how fluid flow is produced within small drops in a well controlled manner in order to create mixing or even inverse mixing (i.e. concentrating) effects. Moreover, oscillations of discrete drops bound between two solid substrates are studied. In the following sections, a comprehensive overview of microfluidics, electroosmosis and electrowetting is presented.

\subsection{Microfluidics}

A few decades ago electronics evolved from huge circuits based on vacuum tubes into small scale semiconducting integrated circuits. In simple words, a calculator that was as huge as a room, now fits in a wrist watch. Nowadays, a similar trend is visible in chemical engineering. Biochemical and chemical analysis and synthesis involving fluids, which originally could be done only on large scales, are more and more integrated into a small device called a microfluidic chip (see Fig. 1.1). Such micro - or even nano - scale devices, in general called 'lab on chip' devices, offer a higher accuracy and better efficiency compared to bulk processes. Microfluidics deals with fluids in the sub micro litre range. This miniaturization offers the possibility to exploit liquid properties which dominate at this scale. In microfluidic transport, inertial effects are negligible while viscous effects play a vital role. Surface effects are also dominant on micro scales because the characteristic geometries of microfluidic devices have a relative large surface area to volume ratio. Therefore, surface effects which are insignificant on large scale fluid manipulation, become important in microfluidics and can be utilized to enhance the efficiency of the processes.

Fluids are mostly described as a continuum in which discrete quantities such as mass and force are expressed in terms of mass per volume or density $\rho$ and body force per volume $f$. The net force on a fluid element results from this body force and from the viscous stresses (force per unit area) acting on the bounding surfaces of that fluid element. The continuum version of the fundamental equation of motion, called the Navier- 


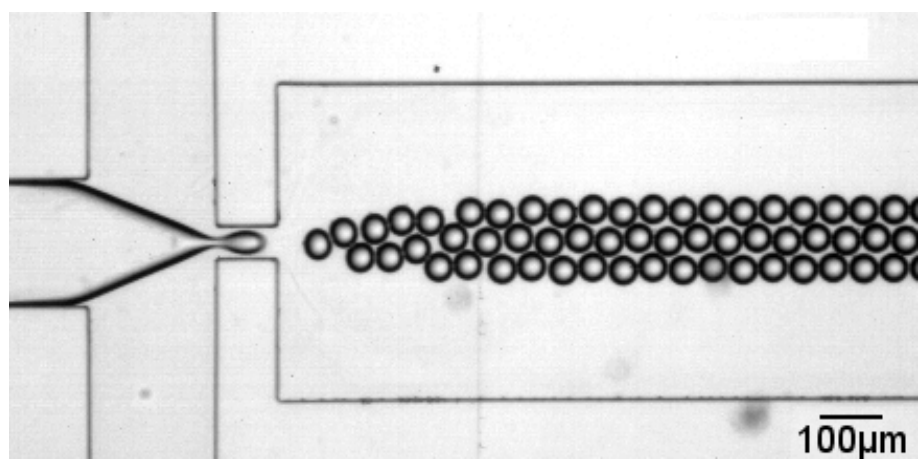

Figure 1.1: A microfluidic chip producing small water droplets in oil for making emulsions. Compared to the bulk process, here the size of the droplets can be controlled precisely. (Courtesy of Hao Gu, PCF, University of Twente)

Stokes equation, gives the velocity field $\underline{u}$ of the fluid motion as:

$$
\rho\left(\frac{\partial \underline{u}}{\partial t}+\underline{u} \cdot \nabla \underline{u}\right)=-\nabla P+\eta \nabla^{2} \underline{u}+\underline{f}
$$

where $P$ is the pressure, $\eta$ the viscosity of the liquid and $t$ the time. For a fluid flowing with velocity $u$, the relevance of inertia and viscosity can be understood by comparing the inertial forces $\left(f_{i} \sim \rho u^{2} / D\right)$ and the viscous forces $\left(f_{v} \sim \eta u / D^{2}\right)$ resulting in a dimensionless Reynolds number (Re $\sim \rho u D / \eta$ ) where $D$ is a typical length scale of the device. In microfluidics this length scale is so small that the flow has a low Reynolds number. Therefore, the nonlinear term in the equation of motion can be neglected, resulting in the time dependent Stokes equation [1],

$$
\rho \frac{\partial \underline{u}}{\partial t}=-\nabla P+\eta \nabla^{2} \underline{u}+\underline{f}
$$

Another important relation is dictated by mass conservation,

$$
\frac{\partial \rho}{\partial t}+\nabla \cdot(\rho \underline{u})=0
$$


In liquids, the molecules are surrounded by other molecules within atomic distance. This makes them incompressible (i.e. $\rho=$ const) due to short range inter-molecular repulsive forces. Hence mass conservation leads to,

$$
\nabla \cdot \underline{u}=0
$$

At the interface of two immiscible liquids, the force balance is a little more complicated. The pressure $p$ (or more precisely the normal stress on the interface) is not continuous but differs by the Laplace pressure $\gamma K_{c}$, where $\gamma$ is the interfacial tension and $K_{c}$ the curvature of the interface at the considered position. In the interface itself, a so called Marangoni stress $\sigma_{m} \sim \nabla \gamma$ can act due to interfacial tension gradients.

In many cases, the flow in microfluidic devices is driven by a pressure gradient. The pressure gradient to maintain a certain flow rate in a channel with typical diameter $D$, scales with $D^{-3}$. Consequently, for small diameters a relatively large pressure gradient is required which can be a hindrance for practical applications. To overcome this problem one can apply an electric force density instead of a pressure gradient. An example is electroosmotic flow (EOF) created by applying an electric field parallel to the axis of the channel, where the flow speed is independent of the size of the channel.

In droplet based microfluidics, discrete droplets are transported through microchannels or moved around over an open substrate. Each droplet can act as an individual micro container for chemicals, biological fluids or even cells. The flow inside such droplets has a large influence on the chemical processes inside it because mixing or stirring within a droplet can enhance reaction processes. To control these mixing flows, again electric fields can be used. In this case electrowetting (EW), i.e. manipulating the contact line of the drop on the substrate by electric forces, is often most suitable.

Because electroosmosis and electrowetting are intensively used in this thesis, the basics of these phenomena are described in the following two sections of this introduction. 


\subsection{Electroosmosis}

\subsubsection{Electric double layer (EDL)}

Solids in contact with liquids bear a surface charge due to the dissociation of surface groups or adsorption of particular ionic species from the liquid on the surface $[2,3]$. For instance, for substrates like glass (silicon dioxide) the surface charge determining ions are hydrogen and hydroxyl ions. Therefore, the surface charge depends on the $\mathrm{pH}$ of the liquid. The mechanism behind this process can be described as follows. At certain $p H_{z c}$ of the bulk solution the surface will be uncharged: point of zero charge. When the $p H$ is increased from $p H_{z c}$ (i.e. the number of $\mathrm{H}^{+}$ions is decreased) a fraction of the hydrogen containing surface groups is dissociated, to compensate for the decrease of $\mathrm{H}^{+}$ions in the liquid [6]. This results in a negative surface charge and a negative surface potential with respect to the bulk. The negative potential in turn increases the proton concentration near the surface and keeps the surface $p H$ close to $p H_{z c}$. Hence, further proton dissociation does not occur and the system reaches an equilibrium.

In general, due to the surface charge, counter ions accumulate near the surface in the liquid under the influence of electric attraction and maintain electro neutrality. This screening region is called the electric double layer (EDL) and contains a surplus counter ions, some of which are bound to the surface, while the others are mobile. The EDL contains the Stern layer, which consists of the inner and outer Helmholtz planes and a diffuse layer where the potential is described by the Gouy-Chapman theory [2, 79]. An illustration of the EDL is given in Fig. 1.2.

In the inner Helmholtz plane the co- and counter ions are not hydrated and are specifically adsorbed to the surface. In the outer Helmholtz plane the ions are partially hydrated and the ion configuration is determined by finite size effects. The outermost, diffusive layer is composed of mobile co- and counter ions. An imaginary slip plane exists in the diffuse layer, where the potential is defined as the zeta potential $\psi_{\zeta}$. In practice, the outer Helmholtz plane and the slip plane are taken as the same. The zeta 


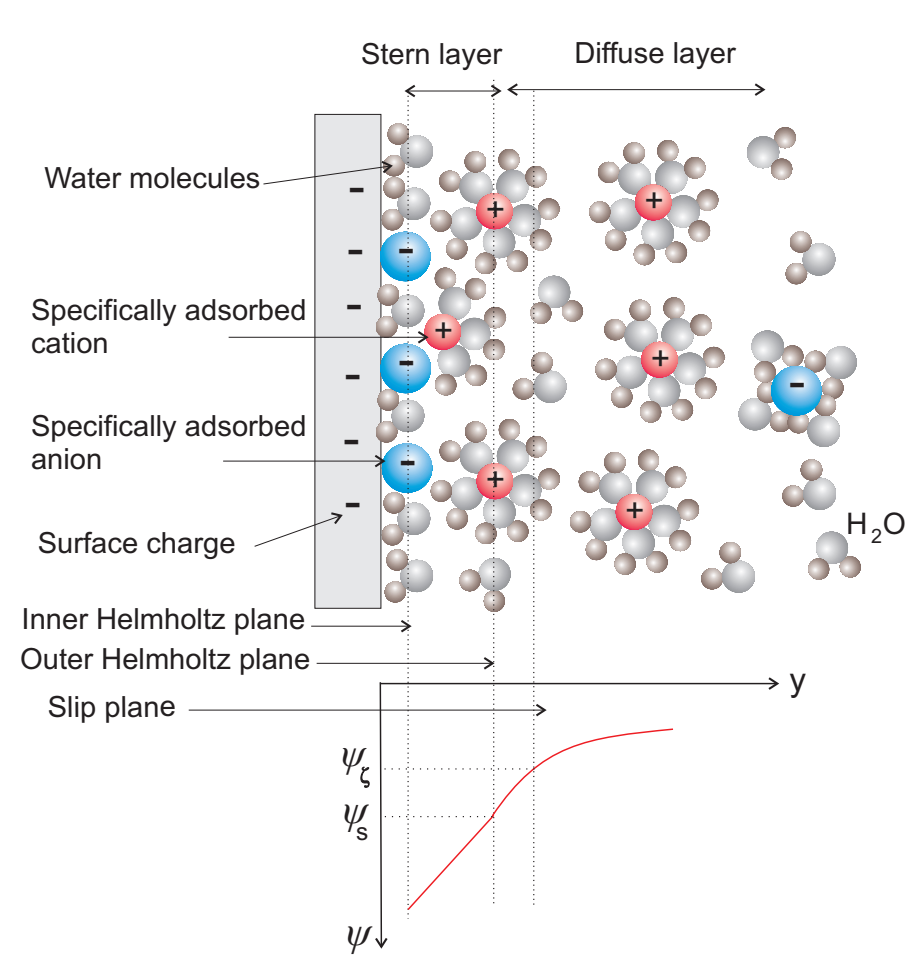

Figure 1.2: Illustration of the electric double layer on a negatively charged solid substrate. The double layer potential is also shown. The potential at the slip plane is called zeta potential.

potential is dependent on the $p H$ and ionic concentration of the solution.

\subsubsection{Potential in the EDL}

To calculate the potential distribution in the EDL, the electrochemical potential $\mu^{e c}$ of an ionic species $i$ at constant temperature and pressure is considered $[7,79]$.

$$
\mu_{i}^{e c}=\mu_{i}+Z_{i} F \psi
$$

where $\mu_{i}$ is the chemical potential, $Z_{i}$ is the valance of the ion $i, F$ is the Faraday constant and $\psi$ is the electric potential due to the surface charge. 
At equilibrium, the electrochemical potential is the same everywhere and the electric and diffusional forces on an ion must be balanced. Therefore,

$$
\nabla \mu_{i}=-Z_{i} F \nabla \psi
$$

The chemical potential can be written as $\mu_{i}=\mu_{i}^{0}+R_{g} T \ln \left(a c_{i} / c_{0}\right)$, where $\mu_{i}^{0}$ is the standard chemical potential of ion $i$ at constant temperature and pressure, $R_{g}$ is the gas constant, $T$ is the temperature, $a$ is the activity coefficient, $c_{i}$ is the molar concentration of ion $i$ and $c_{0}$ is the standard molarity of 1 mol per litre. Integrating Eq.(1.6) from $\psi=0$ at the bulk of the solution (the number concentration $n_{i}=n_{i}^{\infty}$ ) results in the Boltzmann equation giving local number concentration of ion $i$ as,

$$
n_{i}=n_{i}^{\infty} \exp \left(-Z_{i} e \psi / k_{B} T\right)
$$

where $k_{B}=e R_{g} / F$ is the Boltzmann constant. The net charge density at a specific distance from the surface is related to the potential at that position via the Poisson equation,

$$
\nabla^{2} \psi=-\frac{\rho}{\varepsilon_{0} \varepsilon_{l}}
$$

where $\varepsilon_{0}$ is the permittivity of free space and $\varepsilon_{l}$ is the dielectric constant of water. Substituting for the volume charge density $\rho=e \sum n_{i} Z_{i}$, we obtain the Poisson-Boltzmann equation

$$
\nabla^{2} \psi=-\frac{e}{\varepsilon_{0} \varepsilon_{l}} \sum_{i} n_{i}^{\infty} Z_{i} \exp \left(-Z_{i} e \psi / k_{B} T\right)
$$

Eq. (1.9) is a second order elliptic partial differential equation. If $\psi$ depends only on the distance $y$ to the substrate and if the surface potential is small, i.e. $Z_{i} e \psi / k_{B} T<<1$ (Debye-Hückel (DH) approximation), Eq. (1.9) has a simple analytic solution,

$$
\psi(y)=\psi_{s} e^{-\kappa y}
$$

where the Debye-Hückel parameter $\kappa$ is given by,

$$
\kappa=\left(\frac{e^{2} \sum_{i} n_{i}^{\infty} Z_{i}^{2}}{\varepsilon_{0} \varepsilon_{l} k_{B} T}\right)^{1 / 2}
$$


The inverse of $\kappa$ is called the Debye length $\left(\lambda_{D}=\kappa^{-1}\right)$ which is the characteristic thickness of the diffuse layer where the potential decays exponentially.

For a Z-Z electrolyte, Eq.(1.9) can be solved fully to obtain the exact solution. The $\mathrm{DH}$ approximation is within $10 \%$ of the exact solution as long as the surface potential is less than $100 \mathrm{mV}$ (at $25^{\circ} \mathrm{C}$ ) [3]. However at higher potentials, the double layer is thinner than $\lambda_{D}$ and DH approximation is no longer valid. The Gouy-Chapman model and the PoissonBoltzmann equation do not consider the finite size of the component ions [4] and the effect of these ions on the relative permittivity of the solvent [5].

The importance of double layer effects is noticed very commonly in colloidal sciences. The surface charge of particles suspended in a liquid creates a repulsive electrostatic particle particle interaction, besides the attractive van der Waals force. The interplay between the van der Waals and the electrostatic repulsive forces is described by the DerjaguinLandau-Verwey-Overbeek (DLVO) theory [7, 9]. For example, at low ionic strength these forces are responsible for the colloidal stability of a suspension. At high salt concentrations, colloidal particles dispersed in a liquid can aggregate due to the enhanced screening of the electrostatic repulsion.

\subsubsection{Electroosmotic flow}

If an electric field, $E$ is applied parallel to a planar charged surface, the liquid adjacent to the surface is dragged along it. This is called electroosmotic flow (EOF) [11, 76]. The resulting plug flow was first described by [13]. The electroosmotic velocity is zero at the wall (see Fig. 1.3), and increases to a constant value $u_{e o}$ at a distance $\kappa^{-1}$ away from the wall, given by the Smoluchowski equation,

$$
u_{e o}=\frac{-\varepsilon_{0} \varepsilon_{l} E}{\eta} \psi_{\zeta}
$$


The electro osmotic velocity can be measured by monitoring the electric current through the channel as the electrolytes in the channel is exchanged by EOF $[83,107]$. Measuring $u_{e o}$, the zeta potential can be determined from Eq. (1.12).

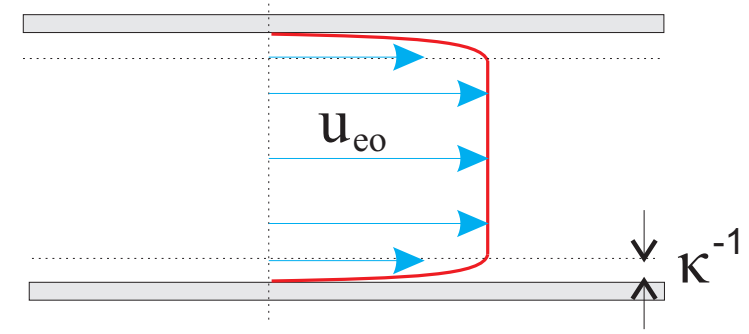

Figure 1.3: The plug flow profile of EOF. In the Debye layer of thickness $\kappa^{-1}$ the flow decreases to zero at the wall.

Within the Debye-Huckel approximation the surface charge $\sigma$ and the zeta potential are connected as

$$
\sigma=\varepsilon_{0} \varepsilon_{l} \kappa \psi_{\zeta}
$$

The surface charge (and so the zeta potential) depends on the ionic composition and strength of the electrolyte, too. This aspect is considered in more detail in Appendix A.

\subsubsection{Electric modification of $\psi_{\zeta}$}

When a small voltage is applied to an otherwise neutral electrode in contact with an electrolyte, again an EDL is formed [10]. This double layer will act as a leaking capacitor. Fig. 1.4 shows the current through a $\mathrm{Pt}$ electrode dipped in $\mathrm{KCl}$ electrolyte when the voltage with respect to the bulk liquid is swept from -3 to $3 \mathrm{~V}$, starting from $0 \mathrm{~V}$. At very low voltages the current-voltage relationship is not linear due to double layer effects. When the voltage is increased, the current increases and it's strength is determined by Faradaic reactions. 
To avoid Faradaic reactions (i.e. electrolysis), the electrodes can be covered with an insulating dielectric layer. Such an embedded electrode is used to control the zeta potential and is often called "modification of the zeta potential by a gate electrode".

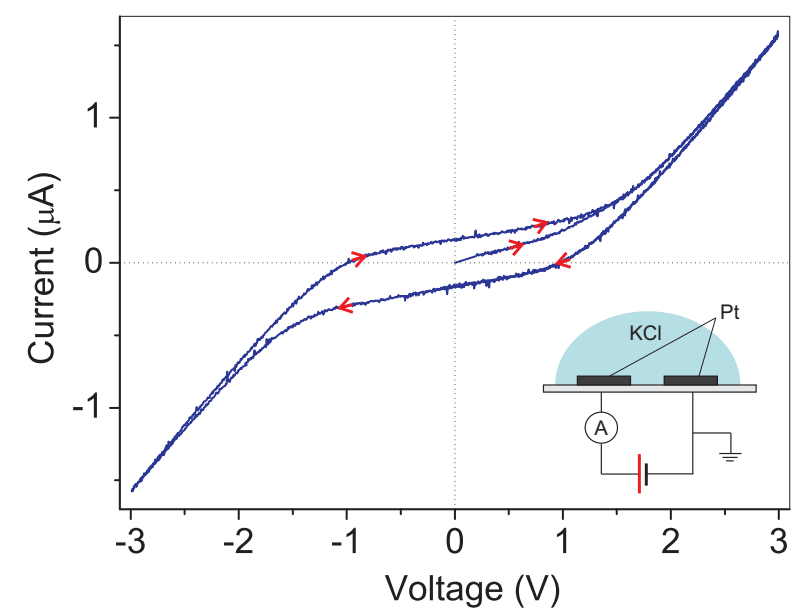

Figure 1.4: The current-voltage curve for a platinum electrode in $\mathrm{KCl}$ electrolyte. The experimental setup is shown in the inset. At low voltages the electrode polarization suppresses Faradaic reactions.

The applied voltage $V$ is distributed between the insulating layer over the electrode and the EDL in the liquid as illustrated in Fig. 1.5. The boundary condition for the perpendicular component of the electric field is given by,

$$
\varepsilon_{l} E_{l}-\varepsilon_{r} E_{r}=\sigma / \varepsilon_{0}
$$

where $E_{r}$ is the field in the insulating layer and $E_{l}=(-\partial \psi / \partial y)$ is the field in the liquid; $\varepsilon_{r}$ and $\varepsilon_{l}$ are the dielectric constants of the insulating layer and the liquid and $\sigma$ is the free charge density on the interface.

Assuming that the potential in the bulk is $\psi_{\infty}=0$, Eq.(1.14) can be rewritten as,

$$
C_{D} \psi_{b}-C_{w}\left(V-\psi_{b}\right)=\sigma
$$




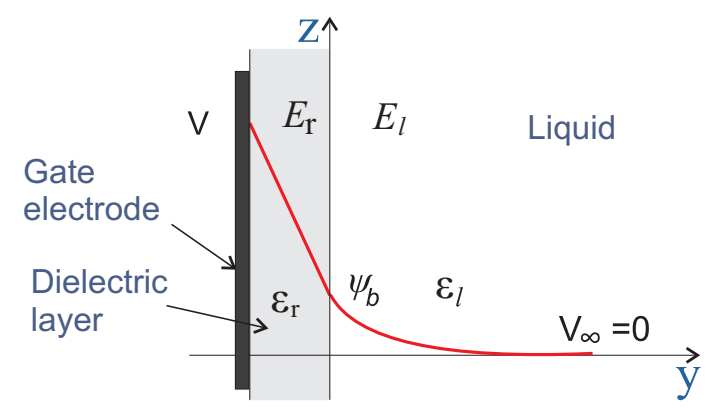

Figure 1.5: The interface between the dielectric layer and the liquid. Most of the voltage $\mathrm{V}$ is dropped in the dielectric layer and the rest in the EDL causing a change in the zeta potential.

where $C_{w}=\varepsilon_{0} \varepsilon_{r} / d$ is the capacitance per unit area of the insulating layer having thickness $d$, and $C_{D}$ is the diffuse layer capacitance ${ }^{1}$ which for a $\mathrm{Z}-\mathrm{Z}$ electrolyte is given by,

$$
C_{D}\left(\psi_{b}\right)=\varepsilon_{0} \varepsilon_{l} \kappa\left(\frac{2 k_{B} T}{e Z \psi_{b}}\right) \sinh \left(\frac{e Z \psi_{b}}{2 k_{B} T}\right)
$$

From Eq. (1.15), the total surface potential when the gate voltage applied is,

$$
\psi_{b}=\frac{C_{w}}{C_{D}+C_{w}} V+\frac{\sigma}{C_{D}+C_{w}}
$$

When no gate voltage is applied, $C_{D}=C_{D}^{\prime}$ with $C_{D}^{\prime}=\varepsilon_{0} \varepsilon_{l} \kappa\left(2 k_{B} T / e Z \psi_{\zeta}\right) \sinh \left(e Z \psi_{\zeta} / 2 k_{B} T\right)$ where $\psi_{\zeta}$ is the intrinsic zeta potential of the wall. Therefore at zero gate voltage, neglecting $C_{w}$ (because $C_{w} \sim 10^{-3} C_{D}$ ), the second term in the right hand side of

\footnotetext{
${ }^{1}$ The differential capacitance per unit area of the diffuse layer is given by $\frac{\varepsilon_{0} \varepsilon_{l}}{\lambda_{D}} \cosh \left(\frac{e Z \psi_{b}}{2 k_{B} T}\right)$. The total capacitance of the EDL includes the capacitances of the Stern layer and the diffuse layer. However, in general the Stern layer capacitance is very high compared to that of the diffuse layer and does not contribute much to the total capacitance. Therefore, the EDL capacitance is nearly equal to that of the diffuse layer especially at low ionic concentrations.
} 
Eq.(1.17) becomes $\sigma / C_{D}^{\prime}=\psi_{\zeta}$. Rewriting Eq.(1.17),

$$
\psi_{b}=\frac{C_{w}}{C_{D}} V+\psi_{\zeta}
$$

In Fig. 1.6, $\psi_{b}$ is plotted as a function of $V$ taking $\psi_{\zeta}=0$. The change in surface potential with gate voltage is not linear at high voltages due to the increased contribution of $C_{D}$.

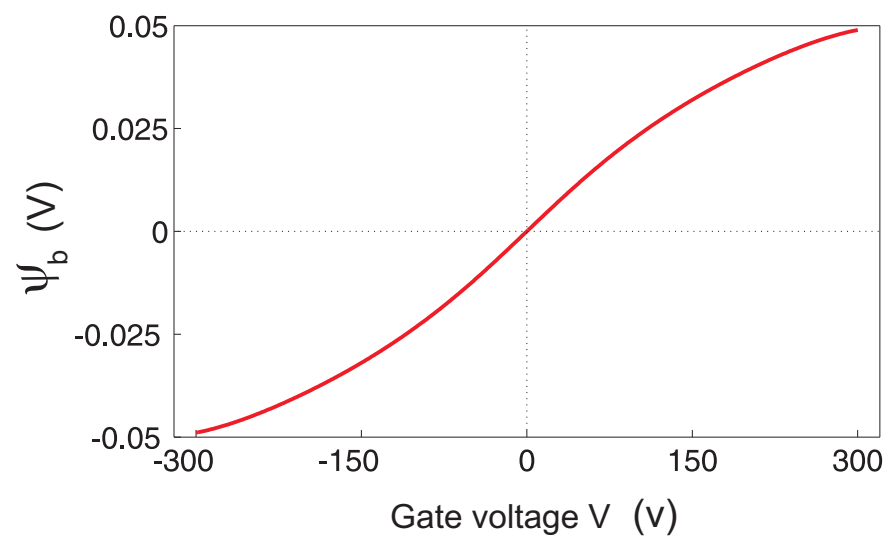

Figure 1.6: The change in zeta potential with gate voltage. The curve is for $1 \mu \mathrm{m}$ dielectric layer with $\varepsilon_{r}=3$ in $1 \mathrm{mM} 1-1$ electrolyte.

In the consideration above it is not taken into account that the dielectric layer can adsorb or desorb protons from the surface groups. When a voltage is applied on the gate, the dielectric layer is polarized which in turn attracts/repels more counter ions from the bulk, increasing/decreasing the zeta potential. Even in the absence of any electric field dielectric layers such as $\mathrm{SiO}_{2}$ can adjust to the chemical environment by proton adsorption or desorption of the surface groups. This is called the buffer capacity of the surface. In the buffering regime, the externally applied field is mostly screened by an increased number of dissociated immobile surface groups. The double layer is consequently left relatively unaffected 
because only a minor shift in zeta potential is required to induce a significant change in $\sigma$ via changes in the activity of $\mathrm{H}^{+}$at the surface [105]. The surface groups continue to dissociate with increasing $E_{r}$ until essentially all are charged. On the other hand, the field over the insulating layer is limited by its dielectric strength.

The above described buffering mechanism (see also appendix A) is undesired in the gate modulation of zeta potential. As a matter of fact, the gate modulation of the zeta potential can only be effective when less number of surface groups are present at the dielectric surface i.e. near $p H$ of the point of zero charge. This adverse effect of buffering can be used to perform titration in a nano confinement [44].

\subsection{Electrowetting}

\subsubsection{Surface tension}

It is commonly observed in nature that water spiders are able to walk on the surface of water without drowning. They make use of the surface tension property of water to balance their weight. Surface tension effects also play an important role in microfluidics due to the relatively large surface to volume ratios involved. In fact the surface tension of an interface is the Gibbs free energy per area at constant temperature and pressure. The molecules at the surface form only less bonds with neighbouring molecules compared to those in the bulk of the liquid. This lack of bonds results in a higher energy for the surface molecules. [16]. The SI units are $\mathrm{J} / \mathrm{m}^{2}$ or $\mathrm{N} / \mathrm{m}$. The surface tension of the water-air interface at $20^{\circ} \mathrm{C}$ is $73 \mathrm{~mJ} / \mathrm{m}^{2}$.

When a liquid is in contact with a solid, the equilibrium forces due to the surface tensions at the liquid-gas $(\gamma)$, solid-liquid $\left(\gamma_{s l}\right)$ and solidgas $\left(\gamma_{s g}\right)$ determine the contact angle of the liquid on the solid [17]. The expression for the contact angle is given by the Young's [18] equation,

$$
\cos \theta_{Y}=\frac{\gamma_{s g}-\gamma_{s l}}{\gamma}
$$


Depending upon the contact angle of water on the solid substrate, the substrate can be classified as hydrophilic $\left(\theta_{Y}<90^{\circ}\right)$ or hydrophobic $\left(\theta_{Y}>90^{\circ}\right)$. For example clean glass is very hydrophilic $\left(\theta_{Y} \sim 0^{\circ}\right.$ in air), while Teflon is hydrophobic $\left(\theta_{Y} \sim 110^{\circ}\right.$ in air).

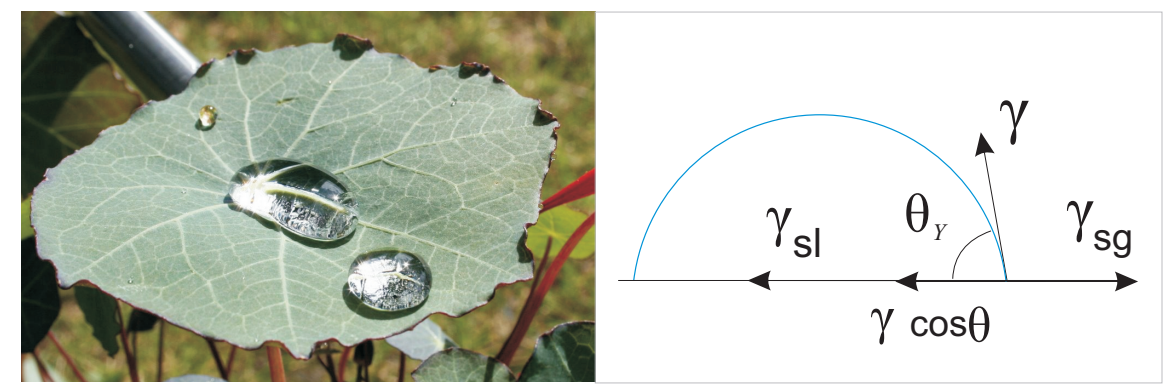

Figure 1.7: Drops try to adopt a spherical shape to minimize the surface energy (see the smallest drop on the leaf). Large drops flatten due to gravity so deviate from the spherical shape (see the two large drops on the leaf). The cartoon shows the surface tension forces at the contact line.

\subsubsection{Effect of electric force at the contact line}

As just stated, the equilibrium contact angle on a substrate results from the competition between different surface tension forces at the triple contact line. The contact angle can be decreased or the wettability can be increased by applying an electrostatic force at the contact line. This force pulls the contact line outwards. In a typical electrowetting setup (Fig. 1.8A), a droplet is placed on a dielectric substrate and a voltage is applied between the droplet and the dielectric layer. The droplet acts as a conductor and spreads over the dielectric layer. Side view images of water drops under electrowetting are shown in Fig. 1.9.

The equivalent electric circuit of the electrowetting setup is a series combination of the dielectric capacitance $C_{r}$ and double layer capacitance $C_{D}$ as shown in Fig. 1.8B. The droplet acts as a conductor with a small 
A

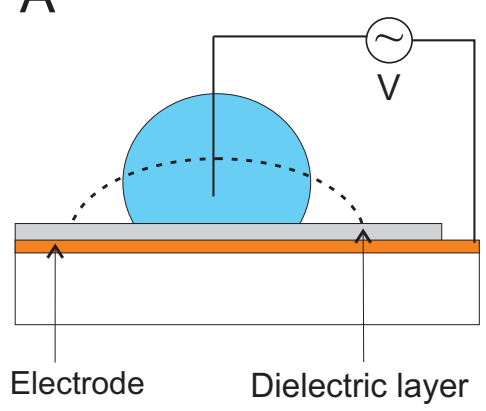

B

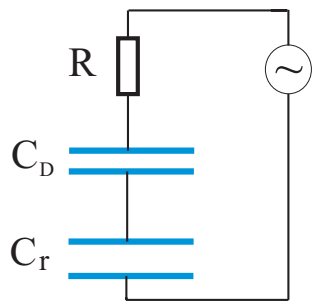

Figure 1.8: A) The experimental setup for electrowetting. B) The electric equivalent diagram of the setup.

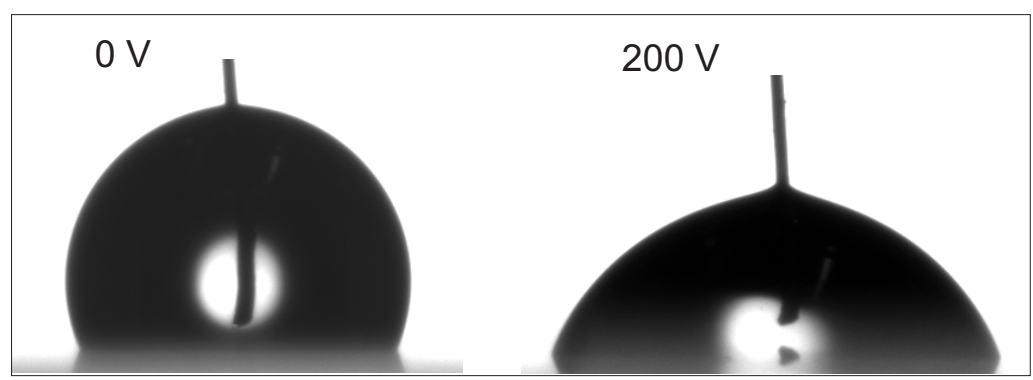

Figure 1.9: Side view images of water drops on Teflon dielectric layer. By applying a voltage (here $200 \mathrm{~V}$ ), electrowetting occurs and the drop spreads over the surface i.e. contact angle decreases. The voltage is applied through a platinum wire inserted in the drop.

resistance $R$. The external fixed voltage source charges the capacitors. The spreading of the droplet increases the capacitance and hence more charge is stored. Since the drop is a conductor, this charge spreads over the surface of the drop and the surface charge density is very high near the contact line of the liquid. Therefore the electrostatic force acting on the liquid surface is concentrated near the contact line. In an electromechanical approach [19] to explain electrowetting, the horizontal com- 


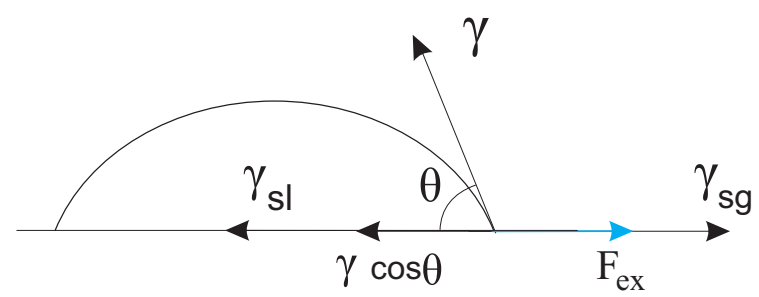

Figure 1.10: The forces due to the Maxwell stress at the contact line are illustrated.

ponent (see Fig. 1.10) of the electrostatic force,

$$
F_{e x}=\frac{\varepsilon_{0} \varepsilon_{r} V^{2}}{2 d}
$$

is equated with the surface tension forces. The horizontal electric force $F_{e x}$ which is independent of the contact angle, pulls the contact line until it balances with the dragging force of the surface tension, $\left(\gamma \cos \theta+\gamma_{s l}\right)$. The force balance at the contact line can be written as

$$
\gamma \cos \theta+\gamma_{s l}=\gamma_{s g}+\frac{\varepsilon_{0} \varepsilon_{r} V^{2}}{2 d}
$$

Using Eq. (1.19),

$$
\cos \theta=\cos \theta_{Y}+\frac{\varepsilon_{0} \varepsilon_{r} V^{2}}{2 d \gamma}
$$

Eq. (1.22) is called the electrowetting (EW) equation. The second term in the right hand side is known as the electrowetting number, $N_{e w}$. It is important to note that the electric force pulling the contact line is proportional to the square of the voltage. This implies that when electrowetting is performed at $\mathrm{AC}$ voltages, the contact line responds for positive and negative half cycles i.e. two times in a full cycle of the voltage. In other words, when a voltage of frequency $f_{a p p}$ is applied to the drop, the contact line oscillates with a frequency $2 f_{a p p}$. In this case, the average contact angle depends on the root mean square voltage. 


\subsubsection{Extensions of the classical electrowetting theory}

The classical electrowetting theory does not consider the influences of finite surface deformations near the contact line or the EDL. The effects of the EDL are less important compared to those of surface deformations since they play a role in a much smaller scale. In practice, both these aspects hardly affect electrowetting and hence are not taken into account. In this section, a brief discussion on the extensions of classical electrowetting theory is presented. A detailed description on the principles and applications of electrowetting can be found in the ref.[19] and the references therein.

\section{Fine structure of the triple contact line}

The electro-mechanical approach carried out above in the mesoscopic scale, gives a physical picture of electrowetting. In that approach, the impact of the fringe fields on the surface profile in the vicinity of the contact line is ignored. The deformations of the liquid surface near the triple contact line must be considered for a complete treatment of the problem. However, these deformations are significant only in a range which is comparable to the thickness of the dielectric layer [20].

\section{Electric double layer effects}

In general, $C_{r} \ll C_{D}$ and electrowetting is governed only by $C_{r}$. However, the microscopic contact angle is affected by the charge distribution in the EDL $[21,22]$. Being the thickness in the order of nanometres, the charge distribution in the EDL does not affect the observed macroscopic contact angle. Including the effects of the EDL, a corrected electrowetting number $N_{e w}$ can be calculated $[19,23]$ as,

$$
N_{e w}^{c o r r}=N_{e w}\left(\frac{1}{1+\varepsilon_{r} \lambda_{D} / \varepsilon_{l} d}\right)
$$

It is also reported that the EDL can cause a diminished electrowetting response when positive voltage is applied on Teflon dielectric layers [21]. 
The steric effects, i.e. the effects of finite size ions, are significant when the thickness of the EDL is comparable to that of the dielectric layer [23].

\subsection{State of the art}

Observation of electoosmotic flow in capillaries dates back to $19^{\text {th }}$ century [11]. The emergence of microfluidics gave a new face to the notion of EOF opening up the doors for many potential applications. In view of microfluidics, EOF in rectangular microchannels is well studied [24]. Currently, EOF is utilized to pump liquids through micro channels [25, 26] for example, in chromatography [27], DNA manipulation [28] or bio-microfluidics [29]. EOF is also used for mixing in microchannels [30].

Time-varying, inhomogeneous electric double layers induced around electrodes give rise to interesting effects as well. Steady electroosmotic flows can be driven using $\mathrm{AC}$ electric fields [31]. In AC electroosmosis, a pair of adjacent, flat electrodes located on a glass slide and subjected to $\mathrm{AC}$ driving, gives rise to a steady electroosmotic flow consisting of two counter-rotating rolls. AC electroosmosis occurs around electrodes whose potential is externally controlled. Nonlinear electroosmosis or induced charge electroosmosis occurs around isolated and inert (but polarisable) objects with both AC and DC forcing. This nonlinear electroosmotic slip occurs when an applied field acts on the ionic charge it induces around a polarisable surface [32].

The magnitude and direction of EOF can be controlled by varying the zeta potential or the surface charge of the channel walls. Ajdari [33, 34] showed that a net electroosmotic flow could be driven either parallel or perpendicular to an applied field by modulating the surface and charge density of a microchannel. In literature, many methods are described to vary the zeta potential: they include using light [35], surface coating of polyelectrolytes [98, 99] or applying voltage on the gate electrodes [38]. The zeta potential can be enhanced to an effective value $\psi_{\zeta}\left(1+b_{s} / \lambda_{D}\right)$ by 
using hydrophobic or super hydrophobic [39] surfaces due to the large slip length $\left(b_{s}\right)$ on such surfaces $[40,41]$. Super hydrophobic surfaces make use of the surface charge at the air-water interface due to the adsorbed $O H^{-}$ions [42].

One of the most explored methods for controlling zeta potential is applying voltage on the gate electrodes patterned on the channel walls. In microfluidics, this technique is used for mixing $[45,106]$ or as field effect transistors [38, 47]. Such techniques are interesting in various applications in nanofluidics as well. The high surface to volume ratio of nanochannels leads to enhanced electrostatic interaction between ions in the bulk of the electrolyte solution filling the channel and charges on the inner surface of the channel itself. In nanofluidic channels where electric double layers overlap, surface charge causes the concentration of coions to decrease and that of counter-ions to increase to neutralize the surface charge. Gate electrodes are used in such nanochannels to control ion transport through them electrostatically [48], for example, to achieve polarity switching in nanofluidic transistors [49].

A local control over the zeta potential enables to create shear flows in a microchannel [50] based on EOF. By changing the zeta potential with voltage, the shear rate can be controlled. Such a device can work as an in situ microrheometer for lab on chip applications. In this thesis, the possibilities for such an EOF based rheometer are explored.

In contrary to continuous flow microfluidics, droplet based microfluidics involves guiding discrete droplets on special substrates. The discrete droplets are used as compartments for cells [51] or reaction chambers for chemicals [52]. Electrowetting can be used to generate droplets in microchannels $[53,54]$ or to transport them on substrates. Electrowetting with AC voltage oscillates the drop at many shape modes [55, 56, 57]. Moreover, these oscillations generate flows inside the drop [58, 59, 60]. Such flows are observed in vertical [61] or horizontal [60] plane depending upon the experimental conditions. The origin of the flows in horizontal plane is not well understood. At very high frequencies of the applied voltage, electrothermal flows are generated inside the drop $[62,63]$ when 
electric fields act on the thermally induced conductivity and permittivity gradients.

These internal flows can be applied, e.g. for microscale mixing [61], or for suppressing particle accumulation at the contact line (coffee stain effect) during evaporation of sessile drops [64]. Coffee stains ${ }^{2}$ are formed when a sessile drop containing non-volatile solutes evaporates while its contact line is pinned on the substrate. When the contact line is pinned at surface defects, mass conservation requires that additional solvent is transported from the center of the drop towards the contact line. This flux carries particles along and deposits at the contact line, ultimately forming a ring shaped solid residue. AC electrowetting can cause the contact line to depin from the surface defects $[65,66]$. Moreover, the internal flows can minimize the particle accumulation at the contact line. These properties are exploited to suppress the coffee stain effect using electrowetting. However, different factors such as the initial solute particle concentration, their size and the applied voltage, which influence the process are to be investigated. Also by understanding the flow mechanism, above mentioned applications can be fine tuned.

\subsection{Outline of the thesis}

This thesis describes studies of two broad topics, i.e., electrokinetics and electrowetting. The experimental techniques used for the studies and their characterizations are described in chapter 2. In chapter 3 we present a simple model to find the surface charge or zeta potential of microfluidic channels. In chapter 4, we explain how shear flow can be created in a microchannel by modifying zeta potential using chemical coating or by applying voltage on the gate electrodes embedded on the walls. In part $I$ of chapter 5, we study the flow patterns inside a sessile drop under AC electrowetting. We also explore two different applications of these

\footnotetext{
${ }^{2}$ The word coffee stain or coffee ring does not necessarily refer to the residue pattern formed from coffee drop but to residue pattern formed after evaporation of any sessile drop.
} 
flow patterns. They include sample pre-concentration in sessile drops (part II, chapter 5) and suppressing coffee stain effect (chapter 6). In (chapter 7) we study oscillations of a drop sandwiched between two solid surfaces induced by electrowetting. The thesis ends with the chapter 8 which describes the conclusions of this work. 



\section{Chapter 2}

\section{Experimental techniques}

In this chapter I shortly describe the experimental techniques used in this thesis. The fabrication of microfluidic devices such as microchannels, gate electrodes embedded in the channel walls is discussed as well as the preparation of substrates for electrowetting experiments. In addition, the image acquisition and particle tracking techniques used to measure the flow field inside the microchannel or droplet are described. 


\subsection{Device fabrication}

To study electro osmotic shear flow, microchannels are fabricated with gate electrodes embedded in their walls. By applying a voltage to the gate electrodes the zeta potential of the channel walls can be controlled. The electrodes are insulated with a thin layer of a dielectric material. The procedures followed to fabricate microchannels, gate electrodes and insulation layers are described in this section.

\subsubsection{Microchannels}

To manufacture the microchannels for EOF measurements, three methods were used, i.e. soft lithography, capillary tubes or by cutting channels on Polydimethylsiloxane (PDMS) films, cast onto a silicon wafer. When gate electrodes were included, microchannels were made by soft lithography. To investigate surface charge effects in a microchannels (using solution displacement method) the channels were fabricated with capillary tubes or by casting and cutting of a PDMS film. The following sections delineate the three above mentioned methods of fabricating microchannels.

\section{Soft lithography}

Soft lithography is a low cost method based on replica molding of micro structures. The important ingredient for soft lithography is PDMS (Sylgard 184 and curing agent with a ratio 10:1). After mixing with a curing agent, the liquid like PDMS is degassed to remove all bubbles and poured onto an appropriate mold. The molds are prepared on silicon wafer by pattering SU8 photoresist by UV lithography.

The PDMS on the mold is cured at $60^{\circ} \mathrm{C}$ for one hour. After curing, the PDMS block is removed from the mold. It then contains an engraved replica of the mold (Fig. 2.1A). The cured PDMS is a very flexible and soft, thereby suitable for microfluidic applications. The PDMS block containing a channel replica is bonded to a substrate to form a microchannel with width and height equal to that of the mold. 

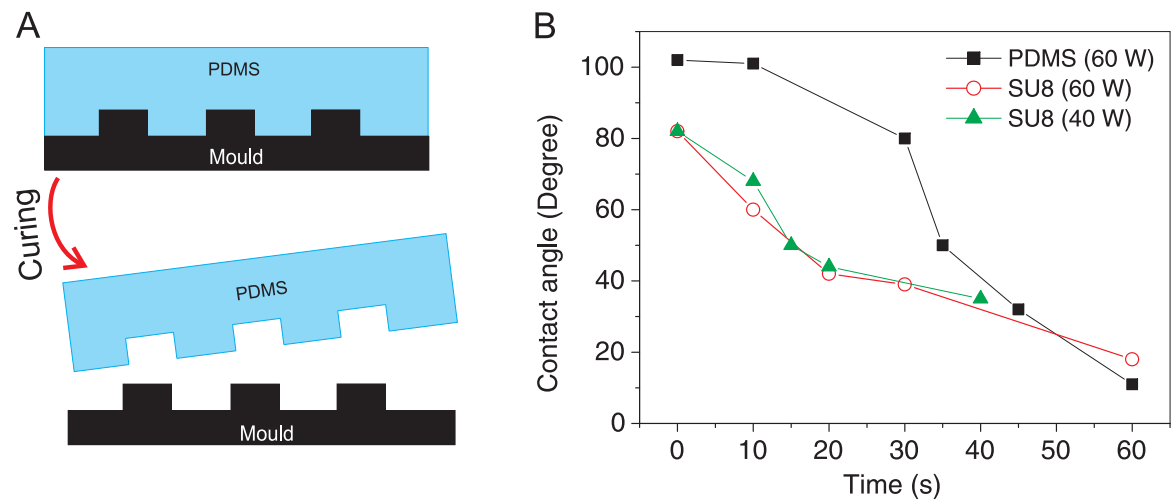

C

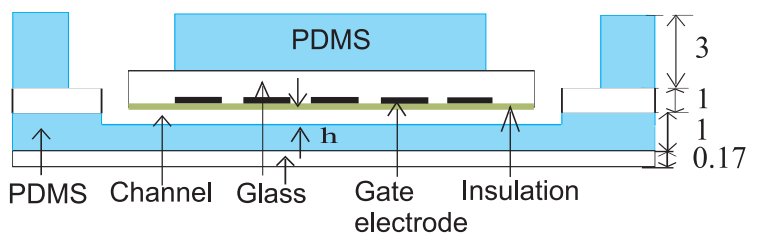

Figure 2.1: A) The principle of soft lithography. B) The contact angle of PDMS and SU8 as a function of the time of plasma treatment. The power used is shown in the inset of B. The uncertainty in the contact angle is \pm 3 degrees. C) Structure of a PDMS channel with upper glass wall containing gate electrodes. Typical dimensions $(\mathrm{mm})$ are also shown.

In the experiments in this thesis, PDMS channels are bonded to glass substrates on which a thin dielectric film is coated with gate electrodes underneath. The structure of such a channel is shown in Fig. 2.1C. The thickness of the PDMS block containing the channel was $1 \mathrm{~mm}$. This is because during measurements the channel has to be viewed through the PDMS block since the gate electrodes are not transparent. A thin glass slide is kept under the thin PDMS block for support. Prior to the bonding, PDMS is treated with oxygen plasma to make it hydrophilic [71] in order to ensure strong bonding between PDMS and the substrate. In Fig. 2.1B, the contact angle of water on PDMS and SU8 is given as a function of the duration of the plasma treatment. After plasma treatment, the contact 
angle is significantly lowered. Moreover, an oxygen plasma treatment of PDMS increases the density of surface silanol $\left(\mathrm{SiO}^{-}\right)$groups [72], which in turn enhances electro osmotic flow velocity.

\section{Using capillaries}

In some of the experiments borosilicate glass capillaries (VitroCom Inc) are used as the microchannels. The typical cross section of the capillary is measured as $43 \times 43 \mu \mathrm{m}$ (within $3 \%$ error). The capillary is fixed between two PDMS blocks. The two openings on the upper PDMS block are used as fluid reservoirs. The space between the PDMS blocks is filled with uncured PDMS and cured to prevent leakage. Fig. 2.2A illustrates the channel with typical dimensions.

\section{By Casting and cutting}

In this technique, the channel is cut on a thin PDMS film. The PDMS film is made on a hydrophobic silicon wafer, by casting the liquid PDMS over it. In the casting procedure ${ }^{1}$, a blade wipes the PDMS on the silicon wafer to form a thin layer of PDMS with a well defined thickness which is determined by the height between the blade and the wafer. The PDMS film on the wafer is cured at $60^{\circ} \mathrm{C}$ for approximately an hour.

A rectangular piece of PDMS film is cut from the wafer and transferred to a piece of Teflon sheet, while keeping the silicon wafer with PDMS in an ethanol bath to prevent coiling of the film. Another piece of Teflon is placed over it to form a Teflon-PDMS-Teflon sandwich. A channel is cut out of this sandwich by impressing it with a double bladed knife as shown in Fig. 2.2C. As the thickness of the PDMS film determines the height of the channel, the distance between the blades determines it's width. After cutting the channel, one of the Teflon layers is removed and the PDMS film is bonded to a glass slide after oxygen plasma treatment.

\footnotetext{
${ }^{1}$ Instead of casting procedure, the PDMS can be also spin coated. The viscous uncured PDMS can be diluted in n-Hexane to obtain thin films after spin coating.
} 

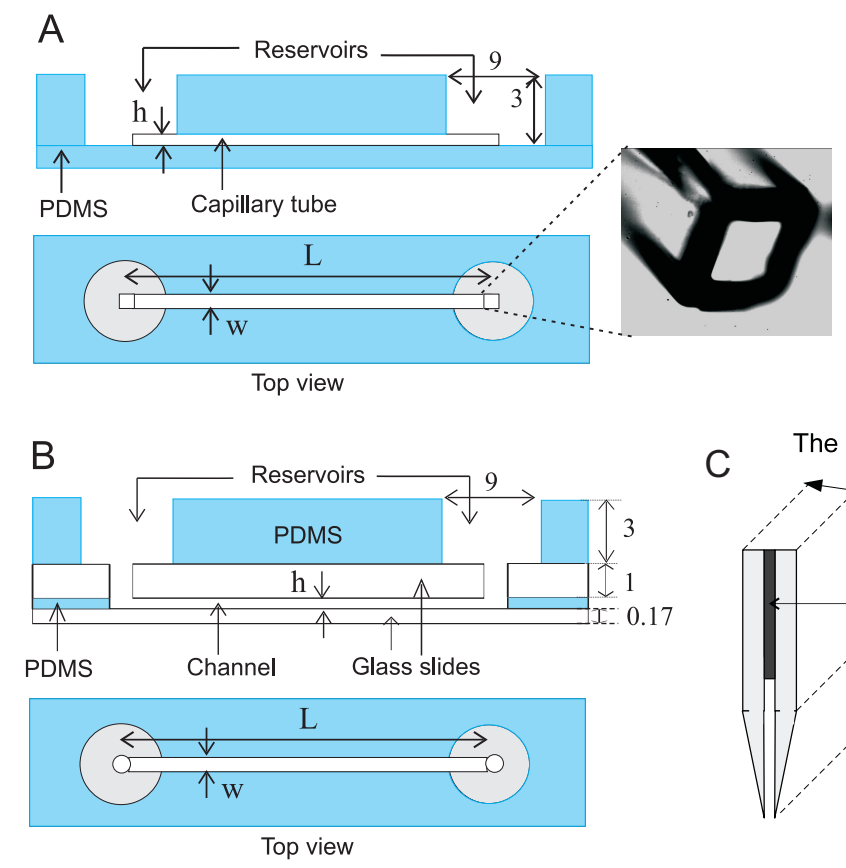

C The blades

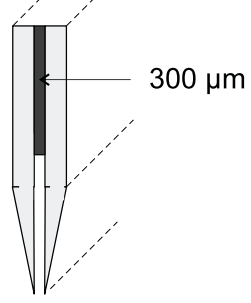

Figure 2.2: The structure of the channel made A) using glass capillaries, B) by casting and cutting. C) The structure of the blade used. Typical dimensions ( $\mathrm{mm}$ ) are also shown.

Next the other Teflon sheet is also removed and a second glass slide is bonded to the PDMS, again after oxygen plasma treatment. The structure of the resulting channel is shown in Fig. 2.2B. The reservoirs on top of the upper glass slide are made with two holes on a thick PDMS sheet.

\subsubsection{Gate electrodes}

The planar electrodes on the walls of the channel to control EOF, are called gate electrodes. In this thesis work, such electrodes are made on borofloat glass substrates using the lithographic process. This includes different steps such as, coating a photoresist, UV illumination, baking 
and etching as illustrated in Fig. 2.3.

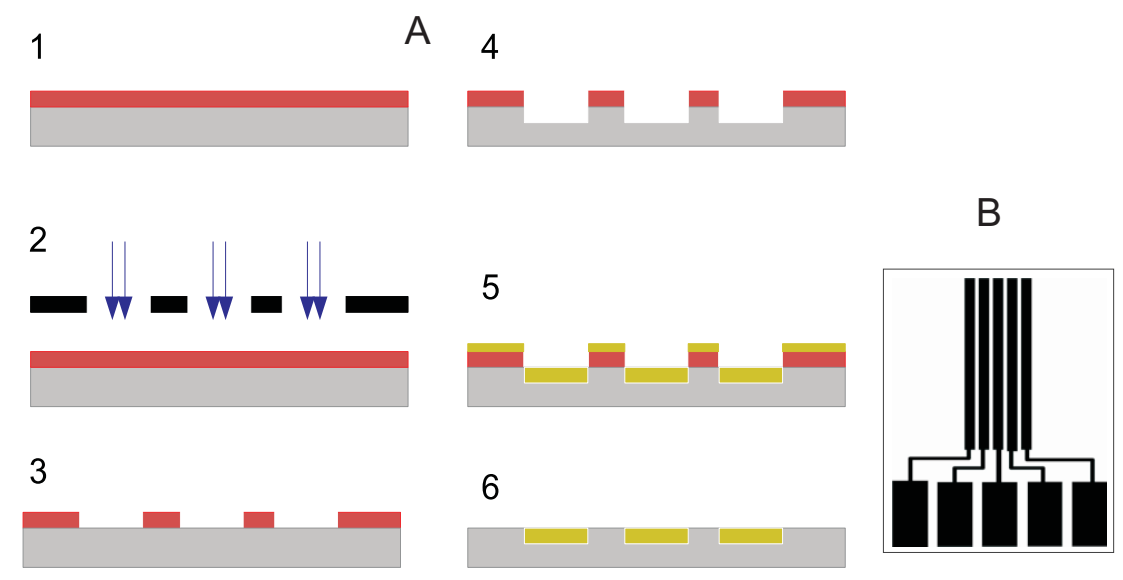

Figure 2.3: A) 1. The glass substrate is cleaned, a positive photoresist is spin coated on it and cured. 2. Using a Cr coated mask, well defined regions of the photoresist are UV illuminated. 3. After a hard bake, the substrate is treated with RER600 solution to etch away the UV irradiated regions. 4. Small grooves are made on the glass substrate by etching in BHF (Buffered Hydrogen Fluoride). 5. Metals (e.g. Cr and W) are coated by sputtering. 6 . The substrate is treated with acetone to etch away the photoresist along with the metals on it, leaving planar electrodes on the glass substrate. B) Structure of the gate electrodes used in this thesis work.

In the above mentioned procedure, the time duration for baking, UV illumination and etching depends on the type and thickness of the photoresist and can be found in the manuals of the corresponding photoresists. By choosing a negative or positive photoresist, one obtains a negative or the positive image of the mask on the substrate after etching with a suitable etchant (step 1 to 3 in Fig. 2.3).

\subsubsection{Insulating the gate electrodes}

During EOF measurements, a voltage is applied to the gate electrodes in order to change the zeta potential of the channel wall. To prevent Faradaic 
reactions, the electrodes must be insulated. Therefore the planar electrodes are coated with a dielectric layer. The insulation layers must be thin and must have large dielectric constant. A thin dielectric layer of large dielectric constant is preferred. However, a thin dielectric layer is prone to breakdown easily in contact with liquids due to the pinholes on it. Some of the dielectric layers tested in this work are described below.

\section{SU8 layer}

SU8 is a negative photoresist which acts as a good dielectric material. It has a dielectric strength of $440 \mathrm{~V} / \mu \mathrm{m}$ [67]. The dielectric constant is between 3 and 4 depending up on the type of SU8. The thin layers $(<1 \mu \mathrm{m})$ of SU8 are found to breakdown easily in contact with electrolytes. This may be due to the presence of pinholes. Thick SU8 layers did not make any noticeable change in the zeta potential during EOF measurements. SU8 layers are therefore not suitable for insulating the gate electrodes for controlling EOF.

\section{Spin on glass}

Spin on glass [68] (SOG 500F) coatings are tested as dielectric layers. After baking at $400^{\circ} \mathrm{C}$, SOG layers have good dielectric properties similar to $\mathrm{SiO}_{2}$. However they showed very low dielectric strength $(<100 \mathrm{~V} / \mu \mathrm{m})$ in contact with electrolytes.

\section{Teflon}

Teflon AF 1600 solution is prepared with 6\% concentration in solvent FC75. The glass substrate containing gate electrodes is dipped in the Teflon solution and retracted at a rate of $15 \mathrm{~cm} / \mathrm{min}$. After baking at $250^{\circ} \mathrm{C}$ for 30 minutes, this resulted in a $3.2 \mu \mathrm{m}$ thick layer of Teflon on the substrate. These layers have a very low breakdown voltage of 60 $\mathrm{V} / \mu \mathrm{m}$ and were poor insulators for gate electrodes.

\section{PECVD grown ONO layers}

High temperature is required for the formation of the $\mathrm{SiO}_{2}$ layers. However, when enhanced by plasma, $\mathrm{SiO}_{2}$ or $\mathrm{Si}_{3} \mathrm{~N}_{4}$ layers can be deposited at lower temperatures by using chemical vapour deposition. The following chemical reaction occurs in a plasma enhanced chemical vapour 
deposition (PECVD) chamber.

$$
3 \mathrm{SiH}_{4}+6 \mathrm{~N}_{2} \mathrm{O} \longrightarrow 3 \mathrm{SiO}_{2}+4 \mathrm{NH}_{3}+4 \mathrm{~N}_{2}
$$

or

$$
3 \mathrm{SiH}_{4}+4 \mathrm{NH}_{3} \longrightarrow \mathrm{Si}_{3} \mathrm{~N}_{4}+12 \mathrm{H}_{2}
$$

PECVD grown $\mathrm{SiO}_{2}$ layers contain many pinholes. Therefore, such layers in contact with liquids have very low dielectric breakdown voltages. By making sandwich layers of $\mathrm{SiO}_{2}-\mathrm{Si}_{3} \mathrm{~N}_{4}-\mathrm{SiO}_{2}$ (hereafter called ONO layers) a better quality is achieved. Annealing the layers at $400^{\circ} \mathrm{C}$ for 24 hours in nitrogen flow improves their quality.

A successful recipe used in this work is described in table 2.1. Choosing the right electrode material is important to get a good dielectric layer over it. Commercial ITO (Indium Tin Oxide) coated glass substrates are found not successful for the PECVD insulation. The electrodes with gold $\mathrm{Au})$ or ITO will deform at deposition/annealing temperatures [69] and damage the dielectric layers. Tungsten (W) which has high melting point is a good candidate for the electrode material.

The PECVD-grown dielectric layers show an asymmetry in the breakdown voltage for negative and positive voltage bias. They withstand only lower negative voltage compared to positive voltage as shown in Fig. 2.4A. This might be due to the charge trapping during the deposition [70]. Moreover, the surface chemistry of PECVD oxide layers is different from that of glass. For example, the surface charge is found to fluctuate with time as shown in Fig. 2.4B.

\subsubsection{Substrates for electrowetting}

Substrates for electrowetting measurements contain a dielectric film coated over a conducting ITO deposited glass. The dielectric layers can be spin coated (e.g. SU8) or dip coated (e.g. Teflon). Substrates coated with SU8 layer of thickness about $5 \mu \mathrm{m}$ are used in the most of the experiments. The 

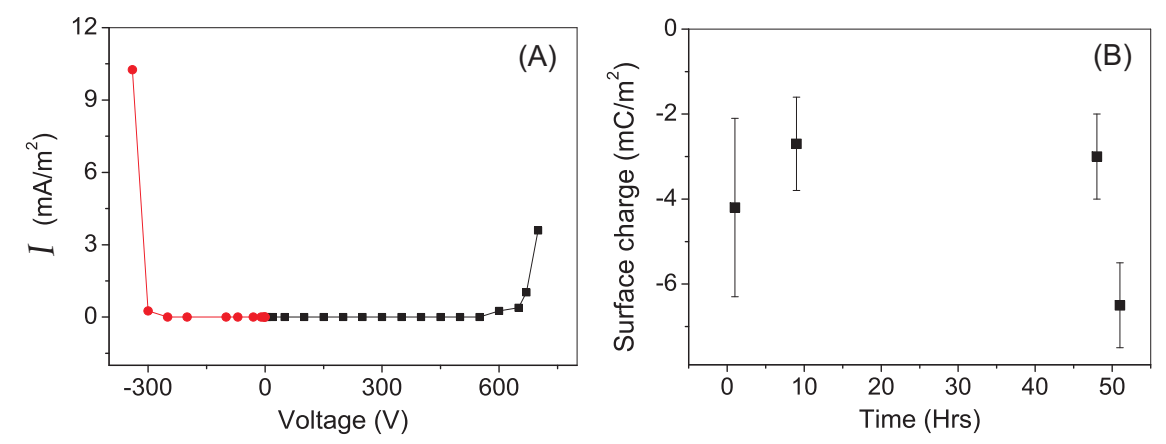

Figure 2.4: (A) A typical current-voltage curve for ONO layer. (B) The surface charge of ONO layer is not very stable over measurements and fluctuates considerably (data for pH 5.6 electrolyte). In between the measurements the channel was kept in deionized water.

\begin{tabular}{llllllll} 
Layer & $\begin{array}{l}\mathrm{SiH}_{4} \\
\text { sccm }\end{array}$ & $\begin{array}{l}\mathrm{NO}_{2} \\
\text { sccm }\end{array}$ & $\begin{array}{l}\mathrm{NH}_{3} \\
\text { sccm }\end{array}$ & $\begin{array}{l}\text { Power } \\
\text { W }\end{array}$ & $\begin{array}{l}\text { Pressure } \\
\mathrm{Pa}\end{array}$ & $\begin{array}{l}\text { Temperature } \\
{ }^{\circ} \mathrm{C}\end{array}$ & $\begin{array}{l}\text { Thickness } \\
\mathrm{nm}\end{array}$ \\
\hline \hline $\mathrm{SiO}_{2}$ & 425 & 710 & - & $20 \mathrm{HF}$ & 1000 & 400 & 150 \\
$\mathrm{Si}_{3} \mathrm{~N}_{4}$ & 1000 & - & 20 & $20 \mathrm{HF}$ & 1000 & 400 & 400 \\
$\mathrm{SiO}_{2}$ & 425 & 710 & - & $60 \mathrm{LF}$ & 1000 & 400 & 150
\end{tabular}

Table 2.1: One of the successful recipes used for ONO layer deposition on Tungsten (W) electrodes. After the deposition, sample was annealed at $400{ }^{\circ} \mathrm{C}$ for $24 \mathrm{hrs}$. The breakdown voltages were $700 \mathrm{~V}$ and $-340 \mathrm{~V}$. ( $\mathrm{sccm}$ : standard cubic centimetres per minute)

contact angle and contact line hysteresis ${ }^{2}$ of SU8 are measured by using 'OCA' setup (Fig. 2.5). This setup consists of a CCD camera, motor controlled syringe system, light source and a platform to place the droplet.

\footnotetext{
${ }^{2}$ The contact line of a drop can pin at the microscopic roughness of a surface. This in turn creates different contact angles for advancing and receding motion of a drop on a surface. The difference between the advancing and the receding angles is called the hysteresis and is a measure of the surface roughness.
} 


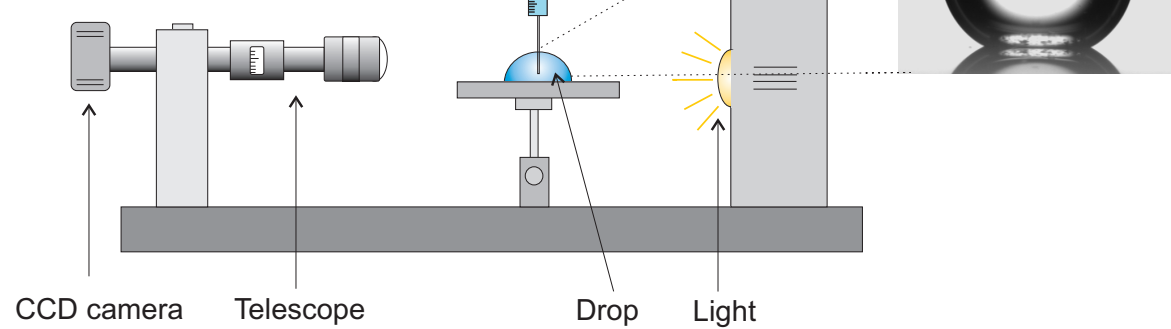

Figure 2.5: The contact angle measurement system. The inset shows an image (side view) of a real drop on a hydrophobic surface.

The contact angle is obtained from the side view of the droplet. The contact angle versus the applied voltage of SU8 is shown in Fig. 2.6A.
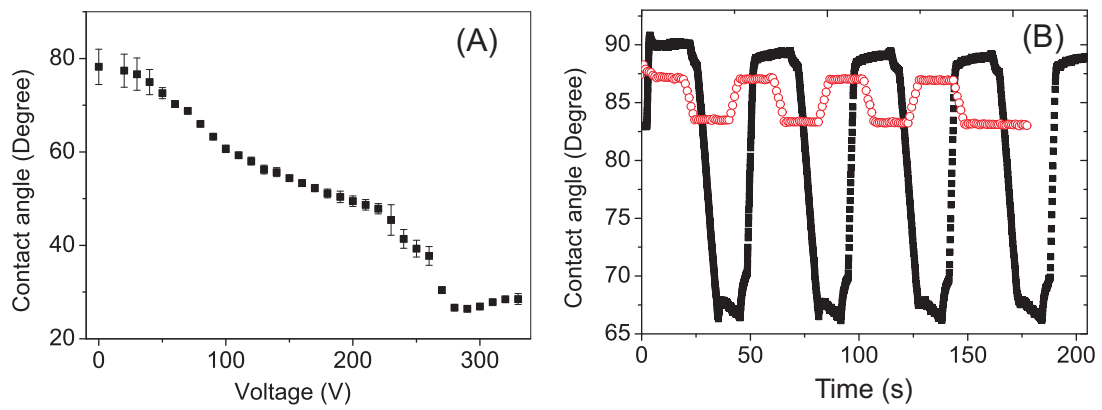

Figure 2.6: (A) The contact angle versus voltage of SU8 surface. (B) The contact angle hysteresis of SU8 (filled squares) is about $25^{\circ}$ and PTCS (open circles) is about $4.5^{\circ}$. The measurements were done in ambient air.

To obtain the hysteresis, the contact angle is measured while pumping the liquid in and out of a drop. Hysteresis is the difference between the advancing and the receding contact angles. SU8 has a large hysteresis of 
about $25^{\circ}$. There are very low hysteresis materials, for example, PTCS ${ }^{3}$ with $4.5^{\circ}$. In Fig. 2.6B, the hysteresis curves of SU8 and PTCS are shown.

\subsection{Imaging and analysis}

In order to study the flow patterns in microfluidics, it is essential to image and analyse local fluid motion. To visualize the local fluid motion, fluorescent tracer particles are put in it. The flow is recorded using video microscopy. The speed and direction of the flow can be determined from the recorded video by tracking the fluorescent tracer particles. The image acquisition and particle tracking methods are described in the following sections.

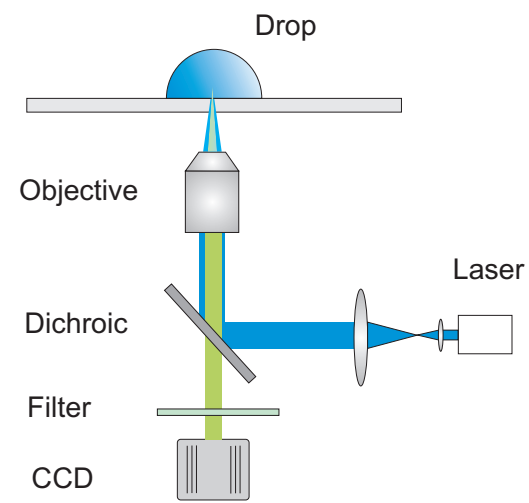

Figure 2.7: Basic instrumentation of fluorescent microscopy.

\subsubsection{Image acquisition}

The fluid flow in microchannels or inside droplets is recorded as video data by using an inverted microscope and a CCD camera (Fig. 2.7). The fluorescent particles in the liquid are excited with a beam of light from a

${ }^{3} 1 \%$ phenyltrichlorosilan (PTCS) in dry toluene is dip coated on silicon wafer. 
laser source. The resulting fluorescent emission is filtered and fed into the CCD camera. A confocal unit is used appropriately with the microscope to image a unique plane of focus to obtain a very sharp image.

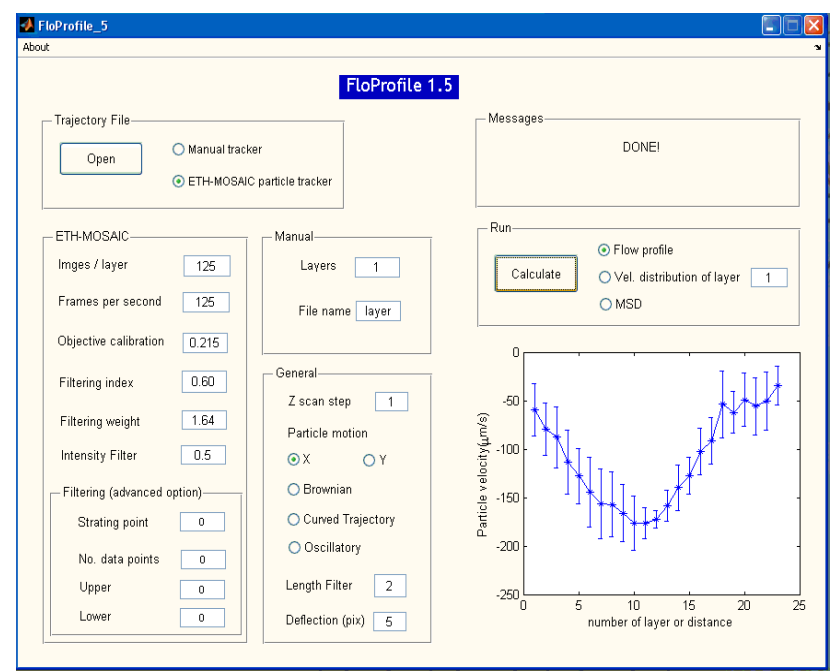

Figure 2.8: The screen shot of the Matlab graphical user interface (GUI) developed to analyse the particle tracking data.

\subsubsection{Particle tracking}

The flow patterns are visualized by putting tracer particles in the working fluid. Particle tracking method is used to determine the velocity of the tracer particles in the fluid. The particle velocity gives the local flow velocity of the fluid in the microchannel or in the drop. The particle tracking is carried out by using the ImageJ plug-in 'ETH-MOSAIC particle tracker' [74]. The trajectory data file obtained from the ETH-MOSAIC particle tracker is analysed by using a home-made Matlab graphical user 


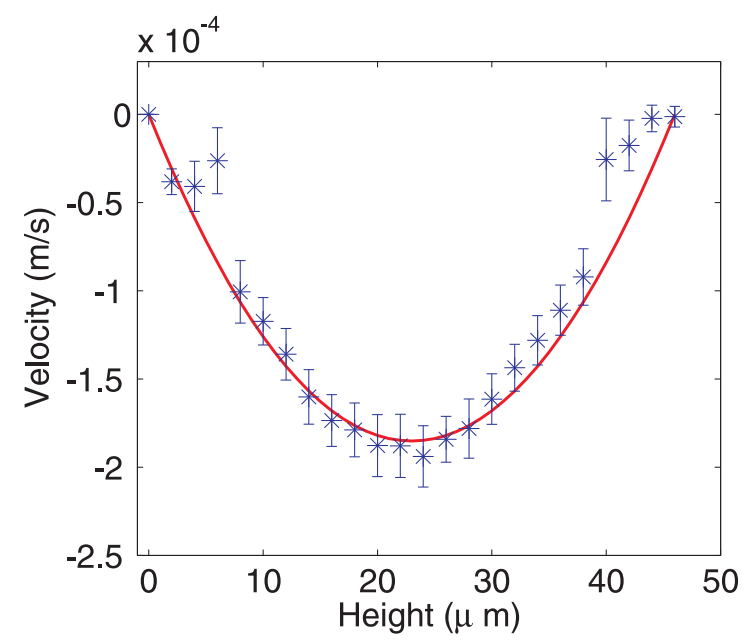

Figure 2.9: The measured and the calculated (line) velocity profiles for a pressure driven flow.

interface (Fig. 2.8) code to determine the particle velocity. Only clear particle trajectories are selected. Noise flickering or particles stuck on the walls are filtered out during the data processing. From the remaining trajectories, particle velocities are calculated from each trajectory, $i$ and averaged over the number of trajectories or over the trajectory length $l_{i}$ as $\langle u\rangle=\sum l_{i} u_{i} / \sum l_{i}$. The trajectory length is taken as a weight factor especially for analysing flows inside a drop. This is because, there are long (near the periphery of a flow vortex) and short (near the center of a flow vortex) trajectories with a wide range of flow speeds. Taking trajectory length as a weight factor will give a typical flow speed in the drop.

For verifying the reliability of the tracking method, a pressure-driven flow is generated with a known pressure head in a channel with known dimensions. The flow velocity at different positions $y$ along the channel height, $u(y)$ is obtained from the recorded data by particle tracking. Additionally, the flow profile is calculated by using the equation $u(y)=-(d P / d L)\left(h^{2}-y^{2}\right) / 2 \eta$, where $h$ is the height of the channel and $(d P / d L)$ is the hydrostatic pressure gradient along the channel. The 
measured and the calculated velocity profiles are plotted in Fig. 2.9. They match very well. It concludes the reliability of the particle tracking procedure. 


\section{Chapter 3}

\section{Determining the surface charge of microfluidic channels}

We study electroosmotic flow through microchannels, made of glass or glass-PDMS, by displacing an electrolyte solution at given concentration with the same electrolyte at a different concentration via an external electric field. When a constant voltage is applied over the channel, the electric current through the channel varies during the displacement process. We propose a simple analytical model that describes the time dependence of the current regardless of the concentration ratio chosen. With this model, which is applicable beyond the Debye-Hückel limit, we are able to quantify the electroosmotic flow velocity and to determine the surface charge on the microchannel walls from the measured current behavior, as well as the zeta potential at given local electrolyte concentration. 


\subsection{Introduction}

Lab-on-a-chip devices and biosensors involve fluid transport through microchannels. This can be achieved by pressure driven flow [75], electroosmotic flow [76, 77, 3] or even by electrowetting [19] principles. The pressure gradient to maintain a certain flow velocity in a channel with typical dimension $D$, scales with $D^{-2}$, but the electric field to maintain the same velocity in electroosmotic flow does not depend on $D$. Hence, for microchannels it is more efficient to drive the flow by electroosmosis. Applying electroosmotic flow one takes advantage of the charged double layer, which is formed on the walls of a solid in contact with an electrolyte due to protonation, deprotonation, specific adsorption of ions or various physicochemical processes such as surface defects or dissolution of functional groups. [3, 78, 79]. To control this flow through a microchannel, one has to know the charge on its walls. Since this surface charge is sensitive to small differences in chemistry and treatment of the surfaces [80], one can not rely on general data. Therefore we developed a new and relatively simple approach to determine the surface charge in situ.

Electroosmotic flow (EOF) can be evaluated using flow visualization [81] or via the so called solution displacement method. In the latter case an electrolyte solution with a given concentration is displaced in the microchannel by the same solution but with a different concentration. During this displacement, the current through the channel changes. This current variation can be used as a measure for the motion of the diffusive boundary between the two concentration regions and hence it monitors the fluid flow velocity. A detailed description of this method can be found in ref. [82]. The solution displacement method in microchannels has been used by Ren, Arulanandam et al. $[83,84]$ to evaluate the average electroosmotic velocity in polyamide coated silica capillary tubes. They used a concentration ratio close to one to ensure that the zeta potential and the net charge density are constant along the channel. Using the average electroosmotic velocity measured by the displacement method, the surface conductance (i.e. the conductance inside the double layer) in silica capillary tubes was determined. In a different study Ren et al. $[85,86]$ 
also modeled and measured mixing effects at the interface between two displacing solutions in a silica capillary. They solved the convectiondiffusion equation numerically in an iterative way, to get the concentration distribution in the mixing zone and the resulting flow velocity. More essential, they took into account the pressure gradient variation inside the channel due to the varying electrolyte concentration and resulting electroosmotic contribution to the flow speed. However, this rather complex model is not easily applicable in practical situations. Wang et al. [87] studied the solution displacement method, theoretically and experimentally, in micro-capillaries of rectangular cross section. They also modeled the displacement based on the convection-diffusion equation to determine the average electroosmotic velocity and zeta potential of the channel. But they do not take into account the varying pressure gradient along the channel, so their model describes the experimental observations only for small concentration ratios.

Unlike the models mentioned above, we report a simple analytical model to evaluate the solution displacement process in microchannels, also at large concentration ratios. The model takes into account the pressure gradient built up due to the difference in electroosmotic flow speed in the high and low concentration regions [88], but it neglects ionic diffusion from the high to the low concentration region. It describes the experimental results quantitatively. With this model the surface charge in the microchannel, the zeta potential and the electroosmotic velocity at given electrolyte concentration (and not only the average velocity as the former models do) can be obtained from the measured time dependence of the current through the channel, when an electric field is applied along it.

The chapter is further organized as follows. In section 2 the model is described. In section 3 we describe the construction of the microchannels, the solution displacement experiments and how the electrokinetic parameters are obtained from these measurements. In section 4 the results are discussed before the chapter ends in section 5 with the conclusion. 


\subsection{Modeling the channel current}

Consider a microchannel with length $L$, width $W$ and height $h$, connecting two reservoirs as shown in Fig. 3.1. The channel walls are assumed to be uniformly charged with the yet unknown density $\sigma$. Initially, the right reservoir in Fig. 3.1 and the channel contain an electrolyte solution with concentration $C_{\text {init }}$ while the left reservoir contains the same electrolyte but at a concentration $C_{\text {fin }}>C_{\text {init }}$. The electrolyte contains $N$ ionic species, where ions of species $n$ are characterized by their mobility $\mu_{n}$, valency $Z_{n}$ and relative concentration $a_{n}=C_{n} / C$, where $C$ is the concentration of the dominant cation in $\mathrm{mmol} /$ litre $(\mathrm{mM})$. An electric potential difference $V_{\text {ext }}$ is applied across the channel. The time dependence of the current $I$ through the channel is monitored by the potential drop $V=I R_{\Omega}$ over the resistance $R_{\Omega}$. The model neglects diffusion of electrolyte from region 1 with concentration $C_{\text {fin }}$ to region 2 with concentration $C_{\text {init }}$. So a sharp concentration gradient between both regions is assumed. The potential drop over the channel is then given by:

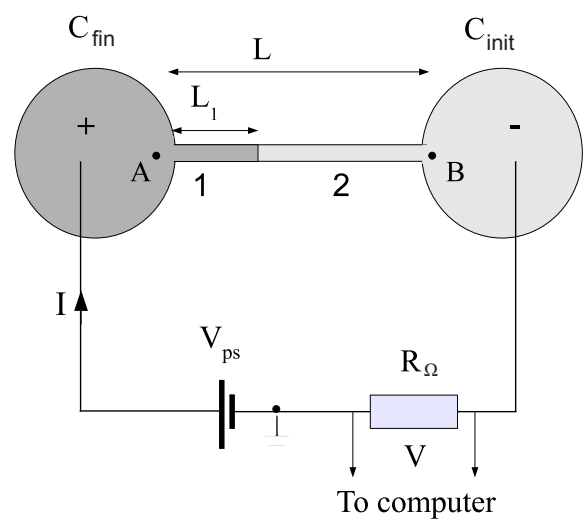

Figure 3.1: The electric connection for solution displacement method for measuring electroosmotic flow (EOF). The electric current in the circuit changes as the solution $C_{\text {fin }}$ enters in the channel due to EOF.

$$
V_{\mathrm{ext}}=E_{1} L_{1}+E_{2}\left(L-L_{1}\right)
$$


where $L_{1}$ defines the length of region 1 and $L-L_{1}$ the length of region 2 . $E_{1}$ and $E_{2}$ are the local axial electric fields in region 1 and 2, respectively. In general, the current $I$ through the channel at electrolyte concentration $C$ is given by:

$$
I=W h e \sum_{n}\left|Z_{n} \mu_{n} a_{n}\right| N_{A} C E
$$

where $e$ is the electron charge and $N_{A}$ Avogadro's number. Because the charge accumulation rate at the boundary between region 1 and 2 is negligible, the current in both regions is the same. But the electric fields are locally different:

$$
C_{\text {fin }} E_{1}=C_{\text {init }} E_{2}
$$

From Eqs. (3.1) and (3.3), one obtains:

$$
\begin{aligned}
& E_{1}=\frac{C_{\text {init }} V_{\text {ext }}}{C_{\text {fin }} L-\left(C_{\text {fin }}-C_{\text {init }}\right) L_{1}} \\
& E_{2}=\frac{C_{\text {fin }} V_{\text {ext }}}{C_{\text {fin }} L-\left(C_{\text {fin }}-C_{\text {init }}\right) L_{1}}
\end{aligned}
$$

and the expression for the current through the channel can be rewritten as:

$$
I(\lambda)=\frac{I_{0}}{1-(1-c) \lambda}
$$

where $I_{0}=W h e \sum_{n}\left|Z_{n} \mu_{n} a_{n}\right| N_{A} C_{\text {init }} V_{\text {ext }} / L$ is the initial current, $c=$ $C_{\text {init }} / C_{\text {fin }}$ the ratio between the initial and final concentration in the channel and $\lambda=L_{1} / L$, the relative length of region 1. If we know $\lambda$ as a function of time $t$, we can calculate the time dependence of the measured current through the channel. To obtain $\lambda(t)$ we calculate $d \lambda / d t$ using the same argumentation as by Devasenathipathy et al. [88].

The electroosmotic driving force depends on the ionic concentration. Because the flow rate through region 1 should be equal to that through region 2, this difference in driving force is compensated by a difference in the pressure gradient $\left[\partial_{z} p\right]_{i}$ in both regions (here and in the sequel the index $i$ refers to region 1 or 2). However, since both ends of the microchannel are at the same pressure, the total pressure drop over the channel is 
zero:

$$
\left[\partial_{z} p\right]_{1} L_{1}+\left[\partial_{z} p\right]_{2}\left(L-L_{1}\right)=0
$$

The velocity of the boundary between region 1 and $2, d L_{1} / d t$, should be equal to mean fluid velocity $u$ which can be written as the sum of the electroosmotic velocity $u_{\mathrm{eo}}=-\varepsilon E_{i} \psi_{\zeta}^{i} / \eta$ and the mean pressure driven velocity $u_{P}$, which is proportional to the local pressure gradient, $u_{P}=\beta\left[\partial_{z} p\right]_{i}$, where $\beta$ is a constant that depends on the geometry of the channel. The relation between the surface charge and the zeta potential for a strong electrolyte is in general given by:

$$
\psi_{\zeta}=\frac{\sigma}{\varepsilon \kappa} g(\sigma, \kappa)
$$

where $\kappa=e\left(\sum_{n} Z_{n}^{2} a_{n} N_{A} C / \varepsilon k_{B} T\right)^{1 / 2}$ is the inverse Debye length, $\sigma$ is the surface charge density on the channel wall, $\varepsilon=\varepsilon_{l} \varepsilon_{0}$ the permittivity of the electrolyte and $g(\sigma, \kappa)$ a (later discussed) function of $\sigma$ and $\kappa$. Hence the electroosmotic velocity in region $i$ can be written as:

$$
u_{\mathrm{eO}}^{[i]}=\frac{-\sigma E_{i}}{\eta \kappa_{i}} g\left(\sigma, \kappa_{i}\right)
$$

where $\eta$ is the dynamic viscosity of the liquid $\left(1 \mathrm{mPa} . \mathrm{s}\right.$ at $\left.20^{\circ} \mathrm{C}\right)$. Hence, the velocity of the interface can be written as:

$$
\frac{d L_{1}}{d t}=\frac{-\sigma E_{1}}{\eta \kappa_{1}} g\left(\sigma, \kappa_{1}\right)+\beta\left[\partial_{z} p\right]_{1}=\frac{-\sigma E_{2}}{\eta \kappa_{2}} g\left(\sigma, \kappa_{2}\right)+\beta\left[\partial_{z} p\right]_{2}
$$

As the surface charge is assumed to be constant (see appendix A), one eventually arrives with Eqs. (3.9), (3.6) and (3.3) at:

$$
\frac{d \lambda}{d t}=\frac{1}{t_{0}} \frac{1-Q \lambda}{1-P \lambda}
$$

where $P=1-c, Q=1-c^{3 / 2} g_{r}$ with $g_{r}=g\left(\sigma, \kappa_{1}\right) / g\left(\sigma, \kappa_{2}\right)$ and:

$$
t_{0}=\frac{-\eta \kappa_{2} L^{2}}{\sigma g\left(\sigma, \kappa_{2}\right)} V_{\mathrm{ext}}^{-1}
$$


Within the Debye-Hückel limit, i.e. at small zeta potentials, $g(\sigma, \kappa)=1$ and so $g_{r}=1$. The time $t_{0}$ is the time it takes a fluid element to pass the channel when it is filled with the solution with initial concentration. Note that $t_{0}$ is inversely proportional to the applied voltage $V_{\text {ext }}$. Integrating Eq. (3.10) results in:

$$
t(\lambda)=t_{0}\left[\frac{P-Q}{Q^{2}} \ln (1-Q \lambda)+\frac{P}{Q} \lambda\right]
$$

With Eqs. (3.5) and (3.12), we calculate the pairs $I(\lambda), t(\lambda)$ for $0<\lambda<$ 1 , i.e. for $0<t<t_{d}$ with $t_{d}=t(1)$ the displacement time. For $t>t_{d}$ the initial solution has been replaced completely by the final solution and the model predicts a plateau value $I_{\infty}$, which scales with the initial current $I_{0}$ and with the applied voltage $V_{\text {ext }}$ according to:

$$
I_{\infty}=c^{-1} I_{0}=\text { Whe } \sum_{n}\left|Z_{n} \mu_{n} a_{n}\right| N_{A} C_{\text {fin }} V_{\text {ext }} / L
$$

In Fig. 3.2 typical examples of the calculated current versus time curves are given for three concentration ratios $c=C_{\text {init }} / C_{\text {fin }}=\left(\kappa_{2} / \kappa_{1}\right)^{2}$ and two values of $g_{r}$. Here $I$ and $t$ have been scaled on $I_{0}$ and $t_{d}$, respectively. We scale the time on $t_{d}$ in stead of $t_{0}$, because doing so the model curves are insensitive to the exact value of $g_{r}$ and we can use $g_{r}=1$ in the fitting procedure without loss of accuracy. The sharp edge near the plateau is not physical because diffusion near the interface between the low and high concentration regions is neglected. The calculated $I(t)$ curves will be fitted to the experimental curves by optimizing $t_{d}$ and $I_{0}$. With the obtained value for $t_{d}$ we eventually determine $t_{0}$ and the surface charge $\sigma$ using Eqs. (3.12) with $\lambda=1$, and (3.11) with $g(\sigma, \kappa)=1$. The corresponding zeta potential and electroosmotic velocity in region $i=$ 1,2 are in the Debye-Hückel limit given by:

$$
\psi_{\zeta}^{i}=\frac{\sigma}{\kappa_{i} \varepsilon}, \quad u_{\mathrm{eo}}^{[i]}=\frac{-\sigma E_{i}}{\eta \kappa_{i}}
$$

For 1-1 electrolytes, as used in this study, the Debye-Hückel approximation is only valid for zeta potentials smaller than $25 \mathrm{mV}$. But for many 


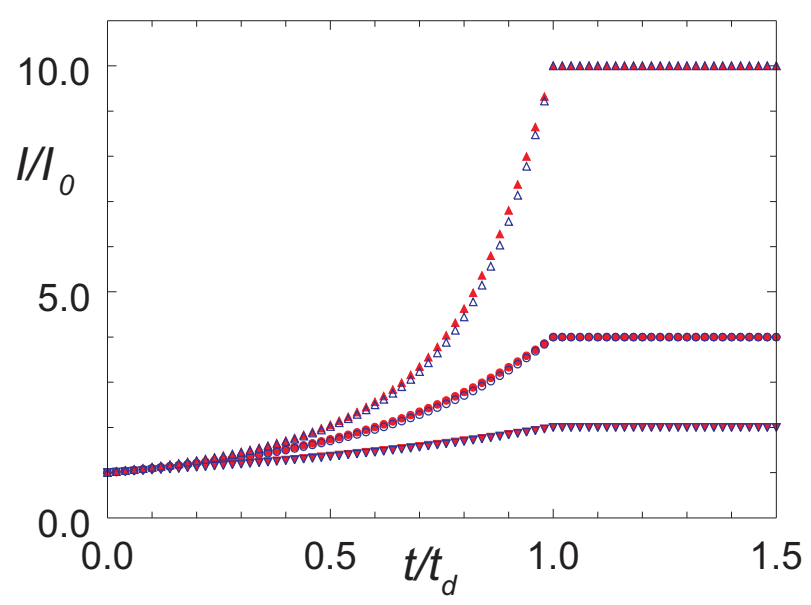

Figure 3.2: The calculated current versus time curves for several concentration ratios $c=0.1(\mathbf{\Lambda}), 0.25(\bullet), 0.5(\mathbf{\nabla})$; full symbols: $g_{r}=1.0$. open symbols: $g_{r}=1.2$.

substrates, including glass, the absolute value of the zeta potential at low ionic concentrations is higher. Therefore we use the Poisson Boltzmann solution [78]

$$
\sigma_{P B}=\frac{2 \kappa \varepsilon k_{B} T}{Z e} \sinh \left(Z e \psi_{\zeta} / 2 k_{B} T\right)
$$

to calcultate the first order corrections for $g(\sigma, \kappa)$ and $g_{r}$ :

$$
g(\sigma, \kappa) \simeq 1-\frac{1}{24}\left(\frac{\sigma Z e}{\kappa \varepsilon k_{B} T}\right)^{2}, \quad g_{r} \simeq 1+\frac{1}{24} P\left(\frac{\sigma Z e}{\kappa_{2} \varepsilon k_{B} T}\right)^{2}
$$

Using these expressions we calculate the corrected values $\sigma_{P B}$ from Eqs. (3.11) and (3.12) and compare them with the initially obtained values for $\sigma$. 


\subsection{Experiments}

\subsubsection{Channel fabrication}

Two borosilicate glass capillaries were used as the microchannel. The cross section of the capillaries was measured as $43 \times 43 \mu \mathrm{m}$ (within $3 \%$ error). One channel (channel 1) was $15 \mathrm{~mm}$ long and the other (channel 2) $12 \mathrm{~mm}$. We also carried out experiments in a channel made of two flat borosilicate glass slides (Menzel-Glaser) separated by a PDMS film from which a rectangle $L \times W$ was cut out (channel 3). The height, width and length of this channel are: $h=36 \pm 4 \mu \mathrm{m}, W=450 \pm 40 \mu \mathrm{m}$ and $L=$ $14.0 \pm 0.1 \mathrm{~mm}$. A detailed explanation about channel fabrication is given in chapter 2. An electric current is applied by two platinum electrodes, inserted in the entrance and exit openings of the channel.

\subsubsection{Current versus time measurements}

We used $\mathrm{KCl}$ (Merck KGaA, Germany) in DI (demineralized) water as electrolyte solution with concentrations ranging from 25 to $250 \mathrm{mM}$, all with a $\mathrm{pH}$ of 5.6. Before starting the measurements, we flushed the capillary with ample amount of DI water. One of the reservoirs and the whole channel are filled with $\mathrm{KCl}$ at a concentration $C_{\text {init }}$ (see Fig. 3.1). The other reservoir is filled to the same level with a $\mathrm{KCl}$ solution at concentration $C_{\text {fin }}$. A DC voltage $V_{\mathrm{ps}}$ is applied using the circuit shown in Fig. 3.1. Due to the large channel resistance, the applied voltage $V_{\mathrm{ps}}$ is only slightly larger (less than $1 \%$ ) than the potential difference $V_{\text {ext }}$ between the entrance A and exit B of the channel, as we checked by inserting two additional electrodes at these positions. Because the reproducibility of the measurements is within a few procents, we neglect this difference.

In channel 3 we used $\mathrm{NaCl}$ (Merck KGaA, Germany) in DI water with ionic concentration 25 and $50 \mathrm{mM}$ (pH about 5.6) as electrolyte.

The current $I$ is monitored by measuring the potential difference $V=$ $I R_{\Omega}$ across the wire-wound resistor with $R_{\Omega}=10 \mathrm{k} \Omega$, using a PC equipped 
with a data acquisition card (National Instruments). The current versus time curves, $I(t)$, were measured for several voltages and concentration ratios, each curve at least three times to check for reproducibility. The values of the initial current $I_{0}$ and the final current $I_{\infty}$ are also measured in separate reference experiments by filling both reservoirs and the channel with the same concentration $C_{\text {init }}$ or $C_{\text {fin }}$.

\subsection{Results}

\subsubsection{Glass capillaries}

Fig. 3.3A displays the current versus time curves measured in channel 1, when a $100 \mathrm{mM} \mathrm{KCl}$ solution replaces a $50 \mathrm{mM}$ solution at several values of $V_{\text {ext }}$. Also the best fitting model curves are drawn. Both solutions mix at their interface during filling and therefore the initial position of the interface between region 1 and 2 is not well defined. This causes a slightly higher initial current $I_{0}$ than observed in the reference measurements, as described in section 3.2. To correct for this effect, each curve is shifted by an appropriate time interval $t_{\text {shift }}$. We fitted our model to the measured curves, by estimating $I_{0}$, and the displacement time $t_{d}$. Next, in a least square fit procedure the values for $I_{0}, t_{d}$ and $t_{\text {shift }}$ were optimized. Because diffusion effects are not negligible for times near the displacement time, we used in this procedure only data points with current values between $I_{\min }=I_{0}$ and $I_{\max }=\left(I_{0}+3 I_{\infty}\right) / 4$ as well as the plateau data. The choice of $I_{\min }$ and $I_{\max }$ is somewhat arbitrary, but varying $I_{\max }$ from $\left(2 I_{0}+3 I_{\infty}\right) / 5$ to $\left(I_{0}+4 I_{\infty}\right) / 5$ affects the obtained value for $t_{0}$ only within $2 \%$. The results for $I_{\infty}$ and $t_{d}$ are given in Table 1. The values of $t_{\text {shift }}$ (not shown) vary only slightly from curve to curve and range between 1 and $10 \%$ of $t_{d}$.

To check the scaling behaviour, as predicted by Eqs. (3.11) and (3.13), all experimental data, are replotted in Fig. 3.3B as $I / V_{\text {ext }}$ versus $t \times V_{\text {ext }}$. All curves collapse quite well to a single master curve, with a plateau value $I_{\infty} / V_{\text {ext }}=0.16 \pm 0.01 \mu \mathrm{A} / \mathrm{V}$, which is close to the theoretically 

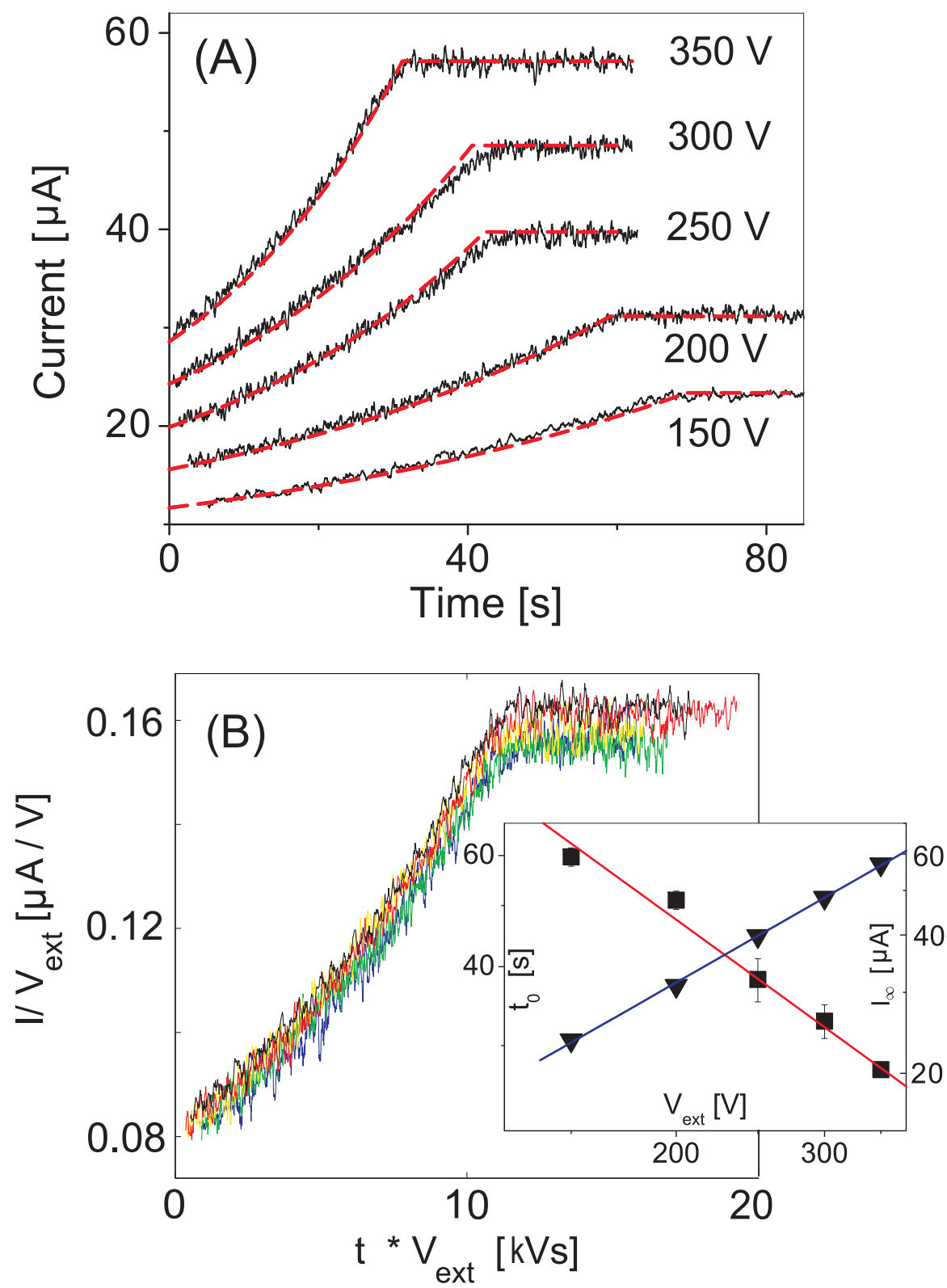

Figure 3.3: A) The current versus time curves measured in channel 1, when a $100 \mathrm{mM}$ $\mathrm{KCl}$ solution replaces a $50 \mathrm{mM}$ solution at several values of $V_{\text {ext }}$. B) All experimental data in (A) are replotted as $I / V_{\text {ext }}$ versus $t \times V_{\text {ext }}$. The values obtained for $t_{0}$, calculated form $t_{d}$ using Eq. (3.12), and $I_{\infty}$ have been plotted versus $V_{\text {ext }}$ in the inset. 
expected value: Whe $\left(\mu_{+}+\mu_{-}\right) N_{A} C_{\text {fin }} / L=0.18 \pm 0.01 \mu \mathrm{A} / \mathrm{V}$. The values obtained for $t_{0}$, calculated form $t_{d}$ using Eq. (3.12), and $I_{\infty}$ have been plotted versus $V_{\text {ext }}$ in the inset of Fig. 3.3B, using logarithmic scales. Fitting a power law to the $t_{0}$ versus $V_{\text {ext }}$ data, one obtains a slope of $-1.00 \pm 0.04$, as expected by the model, and for the $I_{\infty}$ versus $V_{\text {ext }}$ curve a slope of $1.00 \pm 0.04$, which reflects Ohms law.

\subsubsection{PDMS-glass channels}

The displacement curves measured in channel 3 with a $\mathrm{NaCl}$ solution of $25\left(C_{\text {init }}\right)$ and $50\left(C_{\text {fin }}\right) \mathrm{mM}$ are shown in Fig. 3.4A, together with the model curves. These were fitted as described above. Replotting the data as $I / V_{\text {ext }}$ versus $t \times V_{\text {ext }}$, again all curves collapse quite well to a single master curve (Fig. 3.4B). In this case, the plateau value, $I_{\infty} / V_{\text {ext }}=$ $0.57 \pm 0.02 \mu \mathrm{A} / \mathrm{V}$, is slightly smaller than the theoretically expected value, $0.70 \pm 0.10 \mu \mathrm{A} / \mathrm{V}$. This difference can be explained from the rather large uncertainty in the dimensions of channel 3. The inset of Fig. 3.4B shows a $\log -\log$ plot of $t_{0}$ and $I_{\infty}$ versus $V_{\text {ext }}$, from which we obtain the slopes $-1.00 \pm 0.04$ and $1.00 \pm 0.02$, respectively.

Fig. 3.5 shows current versus time curves measured in a square capillary of length $12 \mathrm{~mm}$ (channel 2) at different concentration ratios of the displacing solutions and a fixed voltage $V_{\text {ext }}=300 \mathrm{~V}$. The initial concentration was in all case $25 \mathrm{mM}$, while the displacing concentrations varied from 50 to $250 \mathrm{mM}$. The calculated curves were fitted to the experimental results, using the data points between $I_{\min }=I_{0}$ and $I_{\max }=\left(I_{0}+2 I_{\infty}\right) / 3$ and the plateau data. Again the obtained values for $t_{d}$, given in Table 3.1, are not sensitive to the exact values of $I_{\min }$ and $I_{\max }$. As mentioned before, the mismatch between calculated and experimental curve near the plateau region arises from the ion diffusion at the interface between the two concentrations, which was not taken into account by the model. This effect increases with the concentration ratio. However, the value for $t_{d}$, and hence the surface charge, can be determined by the initial behaviour of the curve. Even for large concentration ratios the displacement time 

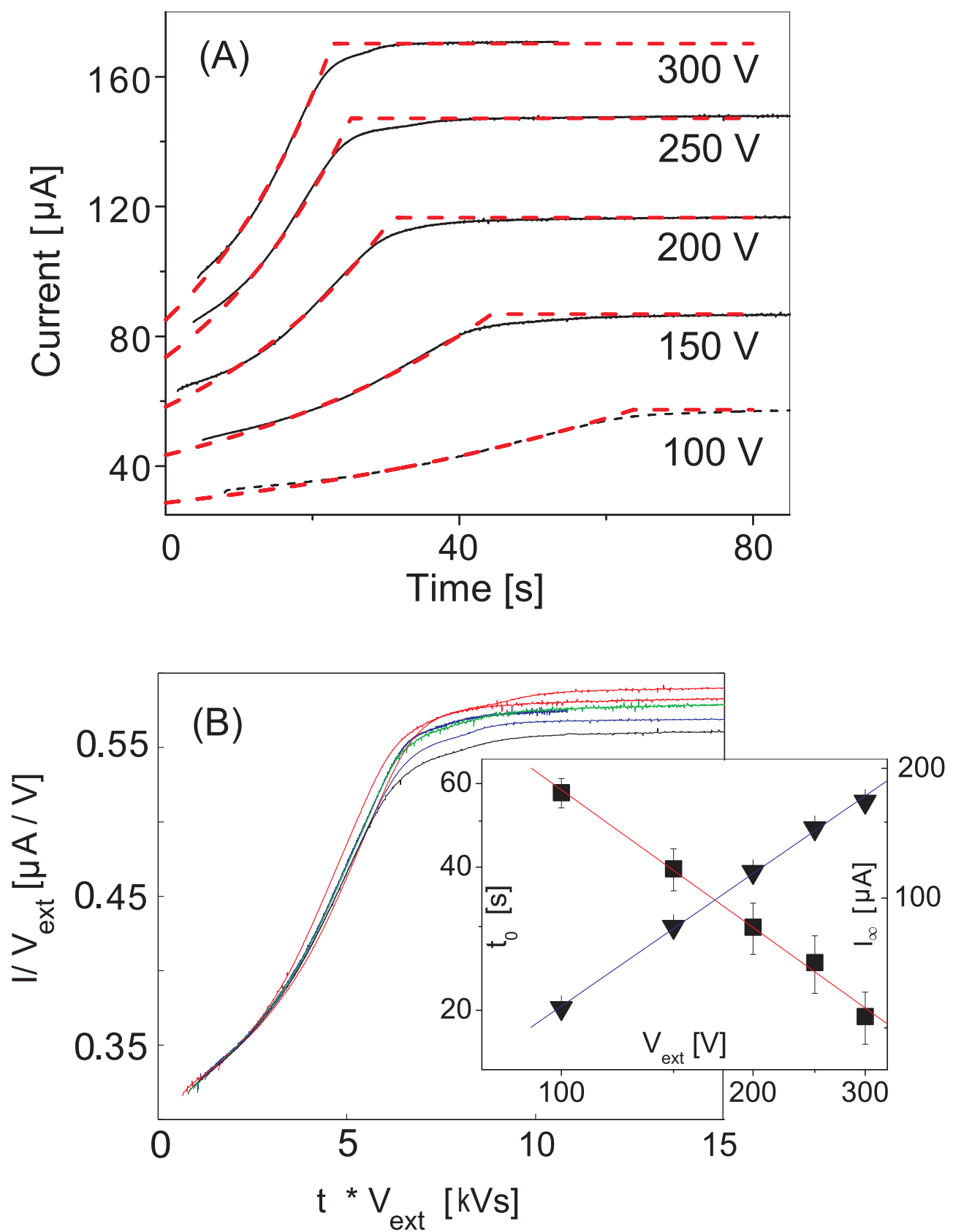

Figure 3.4: A) The displacement curves measured in channel 3 with a $\mathrm{NaCl}$ solution of $25\left(C_{\text {init }}\right)$ and $50\left(C_{\text {fin }}\right) \mathrm{mM}$ are plotted together with the model curves. B) Replotting the data as $I / V_{\text {ext }}$ versus $t \times V_{\text {ext }}$, all curves collapse quite well to a single master curve. 


\begin{tabular}{l|l|l|l|l|l|l|l|l|l} 
Channel & $V_{\text {ext }}$ & $C_{\text {init }}$ & $C_{\text {fin }}$ & $I_{\infty}$ & $t_{d}$ & $-\psi_{\zeta}^{\text {init }}$ & $-\psi_{\zeta}^{\text {fin }}$ & $-\sigma$ & $-\sigma_{P B}$ \\
\hline & $\mathrm{V}$ & $\mathrm{mM}$ & $\mathrm{mM}$ & $\mu \mathrm{A}$ & $\mathrm{s}$ & $\mathrm{mV}$ & $\mathrm{mV}$ & $\mathrm{mC} / \mathrm{m}^{2}$ & $\mathrm{mC} / \mathrm{m}^{2}$ \\
\hline \hline 1 & 150 & & & 23 & $68 \pm 2$ & $36 \pm 1$ & $25 \pm 1$ & $19 \pm 1$ & $20 \pm 1$ \\
\multirow{3}{*}{ glass } & 200 & & & 31 & $58 \pm 2$ & $31 \pm 1$ & $22 \pm 1$ & $16 \pm 1$ & $17 \pm 1$ \\
& 250 & 50 & \multirow{2}{*}{100} & 40 & $43 \pm 3$ & $34 \pm 2$ & $24 \pm 2$ & $17 \pm 1$ & $19 \pm 2$ \\
& 300 & & & 48 & $38 \pm 2$ & $32 \pm 2$ & $23 \pm 2$ & $17 \pm 1$ & $18 \pm 1$ \\
& 350 & & & 57 & $32 \pm 1$ & $33 \pm 1$ & $23 \pm 1$ & $17 \pm 1$ & $18 \pm 1$ \\
\hline 2 & & & 50 & 29 & $15 \pm 2$ & $51 \pm 7$ & $37 \pm 5$ & $19 \pm 3$ & $21 \pm 3$ \\
& 300 & 25 & 100 & 59 & $16 \pm 1$ & $53 \pm 4$ & $27 \pm 2$ & $20 \pm 2$ & $22 \pm 2$ \\
glass & & & 125 & 66 & $19 \pm 2$ & $44 \pm 5$ & $20 \pm 2$ & $16 \pm 2$ & $18 \pm 2$ \\
& & & 250 & 137 & $17 \pm 1$ & $50 \pm 2$ & $16 \pm 1$ & $18 \pm 1$ & $20 \pm 1$ \\
\hline 3 & 100 & & & 56 & $65 \pm 4$ & $48 \pm 3$ & $34 \pm 2$ & $18 \pm 1$ & $20 \pm 1$ \\
& 150 & & & 86 & $46 \pm 4$ & $47 \pm 5$ & $33 \pm 3$ & $17 \pm 2$ & $19 \pm 2$ \\
glass- & 200 & 25 & 50 & 114 & $34 \pm 4$ & $46 \pm 5$ & $33 \pm 4$ & $17 \pm 2$ & $19 \pm 2$ \\
PDMS & 250 & & & 145 & $28 \pm 3$ & $44 \pm 6$ & $31 \pm 4$ & $16 \pm 2$ & $18 \pm 2$ \\
& 300 & & & 168 & $22 \pm 2$ & $48 \pm 6$ & $34 \pm 4$ & $18 \pm 2$ & $20 \pm 2$
\end{tabular}

Table 3.1: Fitted values for $I_{\infty}$ and $t_{d}$ for all experiments, as well as the calculated values for the zeta potential $\psi_{\zeta}$ and the surface charge $\sigma .-\psi_{\zeta}^{\text {init }}$ and $-\psi_{\zeta}^{\text {fin }}$ are the zeta potentials at $C_{\text {init }}$ and $C_{\text {fin }}$ respectively.

$t_{d}$ can be fitted accurately with a relative error of less than about $12 \%$. Overall, the model predicts the current versus time behaviour quite well at all concentration ratio's considered, also for short times.

Once the values of $t_{d}$ have been obtained, the surface charge density $\sigma$, the electroosmotic flow velocity $u_{\mathrm{eo}}$ and the zeta potential $\psi_{\zeta}$ have been calculated using Eqs. (3.11), (3.12) and (3.14). They are also given in Table 1. Since the $\mathrm{pH}$ of the electrolyte solution is the same at all concentrations and no de- or adsorption of ions on the channel walls is expected, the surface charge $\sigma$ is assumed to remain constant during the displacement process, which is consistent with the findings in Table 1. From these data the surface charge density is calculated as $\sigma=-17 \pm 1$, $-18 \pm 1$, and $-17 \pm 1 \mathrm{mC} / \mathrm{m}^{2}$ for channel 1,2 and 3, respectively, independent of the electrolyte concentration. For Fig. 3.5, the calculated values of the surface charge and the zeta potential at the different electrolyte concentrations are given in the inset, where $|\sigma|$ and $\left|\psi_{\zeta}\right|$ have been 


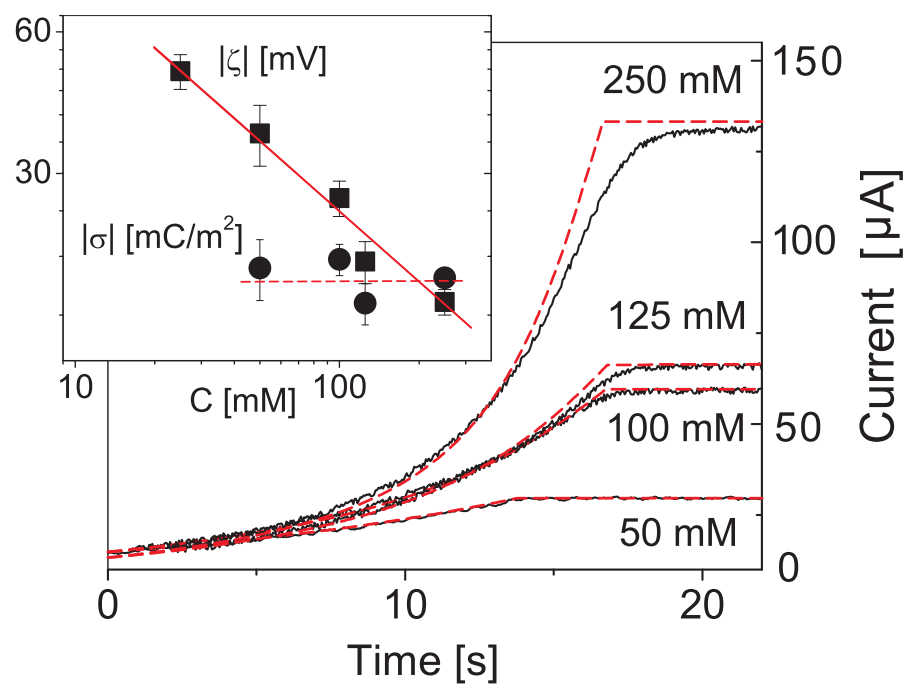

Figure 3.5: The current versus time curves measured in a square capillary of length 12 $\mathrm{mm}$ (channel 2) at different concentration ratios of the displacing solutions and a fixed voltage $V_{\text {ext }}=300 \mathrm{~V}$.

plotted using logarithmic scales. The slope of $\left|\psi_{\zeta}\right|$ versus concentration $C$ is $-0.5 \pm 0.01$ in accordance with the theory while $|\sigma|$ is indeed constant.

\subsection{Discussion}

From Table 1 we observe that the measurements with channel 3 are compatible with those with channel 1: both the zeta potential at $50 \mathrm{mM}$ and the surface charge are within the experimental error the same. This indicates that the PDMS in channel 3, which makes about $7 \%$ of the total wall area, has a negligible effect on the calculated surface charge.

Moreover, we observe from Table 1 that the values for the zeta potential range from 15 to $50 \mathrm{mV}$, which is beyond the Debye-Hückel limit, because $k_{B} T / e \simeq 25 \mathrm{mV}$ at room temperature. Hence, the solution of the nonlinear Poisson Boltzmann equation is considered, too. The obtained 
values for $\sigma_{P B}$ are given in the last column of Table 1. By comparing last two columns we observe differences in $\sigma$ and $\sigma_{P B}$ of about $10 \%$ or less: $\sigma_{P B}=-18 \pm 1,-20 \pm 1$, and $-19 \pm 1 \mathrm{mC} / \mathrm{m}^{2}$ for channel 1,2 and 3 , respectively. The corresponding zeta potential values are in good agreement with those found in literature [89, 3].

The assumption that the surface charge on the walls of the channel is constant during an experiment is quite essential for our analysis. However, the unbuffered electrolyte used in these experiments can create local $\mathrm{pH}$ changes near the electrodes due to electrolysis. In general the surface charge will depend on the $\mathrm{pH}$ of the solution, but the effect of this local $\mathrm{pH}$ changes does not affect the $\mathrm{pH}$ inside the channel due to the large reservoir to channel volume ratio. The fact that $t_{0} V_{\text {ext }}$ is constant during an experiment corroborates this argument. Hence for strong 1-1 electrolytes and bare borosilicate glass, as used in this study, the assumption is valid and the method yields surface charges with an inaccuracy less than $10 \%$. However when using weak electrolyte solutions one should consider the surface chemistry in more detail before one can extract the surface charge from the measured replacement time $t_{d}$.

\subsection{Conclusion}

We have developed a method to determine the surface charge in microchannels in situ, using the solution displacement method. A simple analytical model has been presented to evaluate the time dependence of the measured current during the displacement process. From the obtained displacement time we calculate the surface charge as well as the zeta potential and the electroosmotic flow velocity at given electrolyte concentrations.

Although diffusion effects near the interface between the displacing solutions are neglected, the model is applicable for a large range of concentration ratios, because it does take the pressure variation inside the channel into account. The deviations in the current versus time dependence near the transition to the plateau are quite large for concentration 
ratios larger than four. But the displacement time (and hence the surface charge) can be well determined from the short time dependence of the current and is hardly sensitive to these deviations. Hence, for strong 11 electrolytes, the proposed method is adequate to determine the surface charge in microchannels in a simple way. 



\section{Chapter 4}

\section{Electroosmotic shear flow in microchannels}

We generate and study electroosmotic shear flow in microchannels by chemically or electrically modifying the surface charge and the zeta potential of the channel walls. Chemical modification is obtained by treating one of the channel walls with a cationic polymer. In the case of electric modification we used gate electrodes embedded in one of the channel walls. By applying a voltage to the gate electrodes, the zeta potential can be varied and a controllable, uniform shear stress is applied to the liquid in the channel. The strength of the shear stress depends on both the gate voltage and the applied field which drives the electroosmotic shear flow. Although the stress range is still limited, such a device can be used to shear complex fluids or soft matter in situ in lab on chip devices. 


\subsection{Introduction}

Microfluidic devices are used for chemical analysis of biological fluids or other soft materials [75]. In several applications knowledge of the rheological properties of these fluids is important for the characterization of these materials, for instance in medical diagnostics. Steady or oscillatory shear flow can be used to measure the rheological properties of fluids or to manipulate soft materials in microchannels. It can be used, for example, to stretch DNA molecules [90], to induce conformational changes in proteins [91], or to study the mechanical response of endothelial cells [92].

Moreover, shear flow in microchannels can be used to study the rheology of complex fluids or colloids in confined flow [93, 94]. When sheared in confined geometries, colloidal suspensions can form different shearinduced configurations [95]. Under influence of electric fields the structure of colloidal gels can change, too [96]. When electric fields are not a drawback otherwise, electroosmotic flow (EOF) [3] is a good option to create a shear flow in microchannels.

The magnitude and direction of electroosmotic flow depends on the applied electric field along the flow axis and on the surface charge or zeta potential at the channel walls [3]. When opposing walls are equally charged, EOF results in a plug flow profile. By changing the surface charge or the zeta potential, at one of the channel walls, this plug flow can be transformed to a shear flow. In this chapter, we investigate the possibilities to modify the zeta potential at the channel wall chemically or electrically; in the last case gate electrodes, embedded in the channel wall, are used.

For most materials the intrinsic zeta potential is negative, but it can be decreased or even reversed to positive by coating the channel walls with a cationic polymer. This technique was first introduced in capillary electrophoresis to improve protein analyses [98] and is applied by several other authors [99, 100, 101, 102]. Stroock et al. calculated, and verified experimentally, the flow profile in microchannels with oppositely charged 
surfaces [103].

To control the zeta potential with an external voltage, Schasfoort et al. [38] used a gate electrode which was embedded in the channel wall and covered with a dielectric layer to prevent direct electric contact with the fluid. The potential applied at the gate electrode mainly drops over the dielectric layer but also partially over the Debye layer in the fluid, resulting in an enhancement or reduction of the zeta potential depending on the sign of the applied potential compared to that of the intrinsic zeta potential. As a side effect, this local field enhancement may change under certain conditions the equilibrium between proton adsorption and desorption from the wall, which partially suppresses the change in zeta potential, thereby reducing the effect of the gate potential. For example, in a glass microchannel, the modification of the zeta potential by a gate potential is efficient at low $\mathrm{pH}$ of the electrolyte where the number of surface silanol groups is too low to create buffering effects [105], but is much less efficient at higher $\mathrm{pH}$ values. The principle of controlling the EOF by a gate potential has been used to enhance the mixing effects [106], to modulate the concentration of ions and molecules in a nanochannel [48] or to perform a titration in nano confinements [44]. To our best knowledge, this is the first time gate electrodes are used to generate a uniform shear flow in microchannels. We quantitatively describe the method to create a uniform shear flow in the microchannel and its theoretical background in section 2 . In section 3 the experimental set-up and procedures have been presented. We present our experimental results in section 4 together with a discussion of the efficiency of such an approach and the shear rates that can be achieved in practice. Moreover, some practical issues are addressed, before we conclude our discussion in section 5 .

\subsection{Electroosmotic shear flow}

We consider a rectangular channel of length $L$, with the upper and bottom wall located at $y= \pm h / 2$, the width is $W \gg h$. The channel is filled with a Z-Z electrolyte of which both components have a bulk number density 
$n^{\infty}$. A double layer is formed near the walls due to their surface charge. By applying an electric field $E_{\infty}$ along the axial $z$ direction, the mobile ions in the double layer are dragged by this field and an electroosmotic flow is generated. To understand this mechanism in more detail, we consider the potential in the channel, which is described by the Poisson's equation:

$$
\nabla^{2} \varphi=\frac{-Z e}{\varepsilon_{0} \varepsilon_{l}}\left(n_{+}-n_{-}\right)
$$

where $n_{+}$and $n_{-}$are the number density of positive and negative ions, $e$ the fundamental charge, $Z$ the valence of the ions, $\varepsilon_{0}$ is the permittivity of free space and $\varepsilon_{l}$ the dielectric constant of the liquid. The electric force on an ion due to this potential, $\mp Z e \nabla \varphi$, is balanced by the thermodynamic force, $-k_{B} T \nabla \ln \left(n_{ \pm} / n^{\infty}\right)$, and the hydrodynamic force, $\zeta_{ \pm}\left(\vec{v}_{ \pm}-\vec{u}\right)$, on the ion:

$$
\mp Z e \nabla \varphi-k_{B} T \nabla \ln \left(n_{ \pm} / n^{\infty}\right)=\zeta_{ \pm}\left(\vec{v}_{ \pm}-\vec{u}\right)
$$

where $k_{B}$ is the local Boltzmann constant, $T$ the absolute temperature, $\zeta_{ \pm}$the friction coefficient of the ion, $\vec{v}_{ \pm}$its velocity and $\vec{u}$ the local fluid velocity. Assuming the flow is in the $z$ direction only, the $y$ component of Eq. (4.2) can be solved to get:

$$
n_{ \pm}=n^{\infty} \exp \left(\mp \frac{Z e \psi}{k_{B} T}\right)
$$

where $\psi$ is the potential relative to the bulk potential at axial position $z$ in the channel: $\psi(y, z)=\varphi(y, z)+z E_{\infty}$ with $E_{\infty}$ the external driving field along the axis of the channel. Assuming that $\psi$ depends via its boundary conditions only weakly on $z$ (i.e. $\partial^{2} \psi / \partial z^{2} \ll \partial^{2} \psi / \partial y^{2}$ ) one obtains by substitution of Eq. (4.3) in Eq. (4.1):

$$
\frac{\partial^{2}}{\partial y^{2}}\left(\frac{Z e \psi}{k_{B} T}\right)=\kappa^{2} \sinh \left(\frac{Z e \psi}{k_{B} T}\right)
$$

with $\kappa=Z e\left[2 n_{0} /\left(\varepsilon_{0} \varepsilon_{l} k_{B} T\right)\right]^{1 / 2}$ the inverse Debye length. In our experiments this length is much smaller than the height $h$ of the channel, 
typically $\kappa h \approx 10^{2} \gg 1$, in which case the solution of Eq. (4.4) is given by:

$$
\varphi(y, z)=2 \frac{k_{B} T}{Z e} \ln \left(\frac{1+A(y)+B(y)}{1-A(y)-B(y)}\right)-z E_{\infty}
$$

with $A(y)=A_{0} e^{-\kappa h / 2-\kappa y}$ and $B(y)=B_{0} e^{-\kappa h / 2+\kappa y}$, where $A_{0}=\tanh \left(Z e \psi_{a} / 4 k_{B} T\right)$ and $B_{0}=\tanh \left(Z e \psi_{b} / 4 k_{B} T\right)$ are given by the relative potential at both walls: $\psi_{a}(z)=\psi(-h / 2, z)$ and $\psi_{b}(z)=$ $\psi(h / 2, z)$. For $A, B \ll 1$ Eq. (4.5) reduces to the well known DebyeHückel result:

$$
\varphi(y, z)=\psi_{a} e^{-\kappa h / 2-\kappa y}+\psi_{b} e^{-\kappa h / 2+\kappa y}-z E_{\infty}
$$

To estimate the resulting flow profile inside the channel we consider the momentum balance in the flow direction of a fluid element with volume $d x d y d z$ is given by:

$$
\frac{\partial \tau}{\partial y}=\frac{\partial P}{\partial z}+\varepsilon_{0} \varepsilon_{l} \frac{\partial^{2} \varphi}{\partial y^{2}} E_{\infty}
$$

where $\tau$ is the shear stress in the liquid, $P(z)$ the pressure inside the channel and

$$
-\varepsilon_{0} \varepsilon_{l} \frac{\partial^{2} \varphi}{\partial y^{2}}=Z e\left(n_{+}-n_{-}\right)
$$

the local charge density. By integrating Eq. (4.6) and using Eq. (4.5) one obtains for the shear stress $\tau$ :

$$
\tau(y)=\tau_{0}+\frac{d P}{d z} y+\varepsilon_{0} \varepsilon_{l} E_{\infty} \frac{\partial \varphi}{\partial y}
$$

where $\partial \varphi / \partial y$ is given by:

$$
\frac{\partial \varphi}{\partial y}=4 \kappa \frac{k_{B} T}{Z e} \frac{B(y)-A(y)}{1-(A(y)+B(y))^{2}}
$$

If the liquid in the channel behaves Newtonian with viscosity $\eta$, the shear stress is proportional to the shear rate: $\tau=\eta \partial u / \partial y$. Hence the velocity 
profile can be calculated by integration of Eq. (4.7) using the boundary conditions $u(h / 2)=u(-h / 2)=0$ :

$$
u(y)=u_{p}\left[1-\left(\frac{2 y}{h}\right)^{2}\right]+u_{a}\left(1-\frac{2 y}{h}\right)+u_{b}\left(1+\frac{2 y}{h}\right)+u_{\psi}(y)
$$

with

$$
\begin{aligned}
u_{a} & =-\frac{\varepsilon_{0} \varepsilon_{l} E_{\infty}}{2 \eta} \psi_{a}, & u_{b} & =-\frac{\varepsilon_{0} \varepsilon_{l} E_{\infty}}{2 \eta} \psi_{b} \\
u_{p} & =-\frac{h^{2}}{8 \eta} \frac{d P}{d z}, & u_{\psi}(y) & =\frac{\varepsilon_{0} \varepsilon_{l} E_{\infty}}{\eta} \psi(y)
\end{aligned}
$$

The relative potential $\psi(y)$ is different from zero only inside the double layer, so for $-h / 2+\kappa^{-1} \lesssim y \lesssim h / 2-\kappa^{-1}$ Eq. (4.8) reduces to:

$$
u(y)=u_{p}\left(1-\left[\frac{2 y}{h}\right]^{2}\right)+u_{a}\left(1-\frac{2 y}{h}\right)+u_{b}\left(1+\frac{2 y}{h}\right)
$$

where the parabolic contribution with $u_{p}$ is due to a possible pressure gradient, and the linear contributions are due to the EOF. When $u_{a}=u_{b}$ (i.e. $\psi_{a}=\psi_{b}$ ) Eq. (4.9) represents homogeneous EOF, without a shear component, When $u_{a}=-u_{b}$ (i.e. $\psi_{a}=-\psi_{b}$ ) the shear is maximal and the EOF component of the flow field is zero. The corresponding volumetric flow rate $\dot{Q}$ can be calculated by integrating Eq. (4.9):

$$
\begin{aligned}
\dot{Q} & =W \int_{-h / 2}^{h / 2} u(y) d y=W h\left(\frac{2}{3} u_{p}+u_{a}+u_{b}\right) \\
& =-\frac{W h^{3}}{12 \eta} \frac{d P}{d z}-\frac{W h}{2 \eta} \varepsilon_{0} \varepsilon_{l} E_{\infty}\left(\psi_{a}+\psi_{b}\right)
\end{aligned}
$$

The shear rate in the channel is calculated by differentiating Eq. (4.9):

$$
\begin{aligned}
\dot{\gamma} & =-\frac{8 y}{h^{2}} u_{p}+\frac{2}{h}\left(u_{b}-u_{a}\right) \\
& =\frac{1}{\eta} \frac{d P}{d z} y+\frac{\varepsilon_{0} \varepsilon_{l} E_{\infty}}{h \eta}\left(\psi_{a}-\psi_{b}\right)
\end{aligned}
$$


In an experiment, the velocity profile $u(y)$ can be measured, for instance by particle tracking. The potentials $\psi_{a}$ and $\psi_{b}$ can be measured in situ, for instance by solution displacement method [107].

If the liquid is non-Newtonian the analysis is more complicated. Assuming $d u / d y=f n c(\tau)$ one should integrate Eq. (4.7) numerically and optimise the value for $\tau_{0}$ in an iterative way such that the resulting velocities at the walls are zero.

\subsubsection{Channel with gate electrodes}

Let the upper wall of the channel at $y=+h / 2$ has $N$ equally spaced gate electrodes with equal width $w$. A voltage $V_{n}$ is applied on the $n^{\text {th }}$ electrode. This applied voltage is distributed between the dielectric layer over the electrode and the Debye layer in the liquid as illustrated in Fig. 1.5. The boundary condition for the perpendicular component of the electric fields is given by,

$$
\varepsilon_{l} E_{l}-\varepsilon_{r} E_{r}=\sigma / \varepsilon_{0}
$$

where $E_{r}$ is the field in the dielectric layer and $E_{l}=(-\partial \varphi / \partial y)$ is the field in the liquid, both perpendicular to the wall, while $\varepsilon_{r}$ and $\varepsilon_{l}$ are the dielectric constants of the insulating layer and the liquid, respectively, and $\sigma$ is the free charge density on the interface.

Eq. (4.12) can be rewritten as:

$$
C_{D}\left[\varphi_{s}(z)-\varphi_{\infty}(z)\right]-C_{w}\left[V_{n}-\varphi_{s}(z)\right]=\sigma
$$

where $\varphi_{\infty}(z)=-z E_{\infty}, C_{w}=\varepsilon_{0} \varepsilon_{r} / d$ is the capacitance of the insulating layer having a thickness $d$ and

$$
C_{D}=\varepsilon_{0} \varepsilon_{l} \kappa \frac{2 k_{B} T}{Z e \psi_{s}} \sinh \left(\frac{Z e \psi_{s}}{2 k_{B} T}\right)
$$

the diffuse layer capacitance. The ratio $C_{D} / C_{w}$ is typically $10^{3}$. The net surface potential when a gate voltage is applied can be obtained from Eq. (4.13) and is given by

$$
\varphi_{s}(z)=\frac{\sigma+C_{D} \varphi_{\infty}(z)+C_{w} V_{n}}{C_{D}+C_{w}}
$$




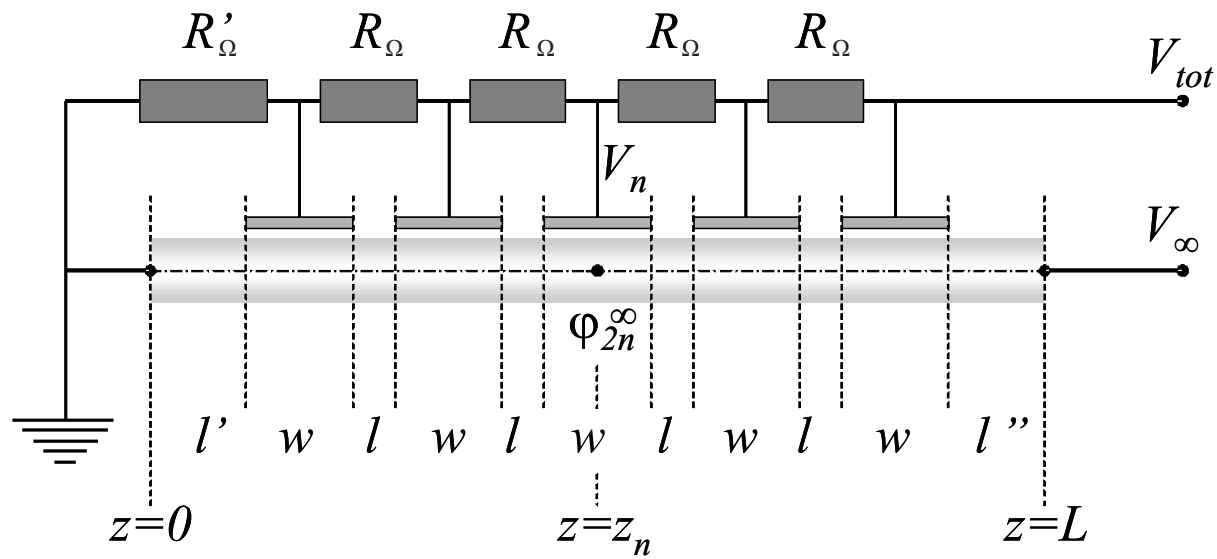

Figure 4.1: Electric circuit with electrode connections.

or with $\sigma=C_{D}^{\prime} \psi_{\zeta}$ where $\psi_{\zeta}$ is the intrinsic zeta potential of the wall at zero gate voltage, and $C_{D}^{\prime}=\varepsilon_{0} \varepsilon_{l} \kappa\left(2 k_{B} T / Z e \psi_{\zeta}\right) \sinh \left(Z e \psi_{\zeta} / 2 k_{B} T\right)$ this is equal to

$$
\psi_{s}(z)=\frac{C_{D}^{\prime} \psi_{\zeta}+C_{w} V_{G}}{C_{D}+C_{w}}
$$

where we defined $V_{G}(z)=V_{n}-\varphi_{\infty}(z)$ as the potential at the gate electrode with respect to the potential in the bulk at axial position $z$. From Eq. (4.14), it is clear that $\psi_{s}(z)=\varphi_{s}(z)-\varphi_{\infty}(z)$ varies along a single electrode due to the potential gradient $-E_{\infty}$ in the channel. To minimize this effect, several electrodes with a width $w \ll L$ are used while we keep $V_{G} \gg \Delta V_{G}=w E_{\infty}$, In section 4 , we evaluate the magnitude of this gradient for the experimental set-up used in this work.

When the voltage $V_{n}$ is applied, the surface potential in the gate region differs from that between two gate electrodes. This inhomogeneity leads to a axial variation of the driving force and hence produces a pressure variation along the channel. To study this aspect in more detail, the channel with length $L$ and containing $N$ electrodes, is divided into $K=2 N+1$ sections, as illustrated in Fig. 4.1. 
The section below electrode $n$, with its center at $z_{n}$ is numbered $k=$ $2 n$ and the section between electrode $n$ and $n+1$ is numbered $k=2 n+1$. Considering a Newtonian liquid, the volumetric flow rate at $k^{\text {th }}$ region, $(1 \leqslant k \leqslant K)$ of the channel can be calculated with Eq. (4.10),

$$
\dot{Q}_{k}=c_{e}\left(\psi_{k}+\psi_{a}\right)+c_{p}\left(-\frac{d P}{d z}\right)_{k}
$$

where $\psi_{k}$ is the surface potential in the $k^{\text {th }}$ region at the upper wall, $c_{e}=-\varepsilon_{0} \varepsilon_{l} E_{\infty} W h / 2 \eta$ and $c_{p}=W h^{3} / 12 \eta$. According to Eq. (4.14) $\psi_{2 n}$ slightly depends on $z$ :

$$
\psi_{2 n}=\frac{C_{D}^{\prime} \psi_{\zeta}+C_{w} V_{n}}{C_{D}+C_{w}}+\frac{C_{w} E_{\infty}}{C_{D}+C_{w}} z
$$

If the pressure difference between the channel ends is $\Delta P=P_{\text {in }}-P_{\text {out }}$,

$$
\sum_{n=1}^{N} \int_{z_{n}-w / 2}^{z_{n}+w / 2}\left(-\frac{d P}{d z}\right)_{2 n} d z+\left[(N-1) l+l^{\prime}+l^{\prime \prime}\right]\left(-\frac{d P}{d z}\right)_{o d d}=\Delta P
$$

where $(d P / d z)_{\text {odd }}$ is the pressure gradient in the (odd numbered) sections without gate electrode. Note that $(N-1) l+l^{\prime}+l^{\prime \prime}$ is just the total length of the sections without electrode. Using Eqs. (4.15) and (4.16), we can express the volumetric flow rate in terms of the surface potentials and the external pressure drop:

$$
\dot{Q}=c_{e}\left(\psi_{a}+\langle\psi\rangle_{b}\right)+c_{p} \frac{\Delta P}{L}
$$

where

$$
\langle\psi\rangle_{b}=\frac{1}{L}\left(\sum_{n=1}^{N} \int_{z_{n}-w / 2}^{z_{n}+w / 2} \psi_{2 n}(z) d z+\left[(N-1) l+l^{\prime}+l^{\prime \prime}\right] \psi_{\zeta}\right)
$$


is the average zeta potential on the upper wall. Using Eqs. (4.15) and (4.17), we find for the local pressure gradient:

$$
\left(-\frac{d P}{d z}\right)_{k}=\frac{c_{e}}{c_{p}}\left(\langle\psi\rangle_{b}-\psi_{k}\right)+\frac{\Delta P}{L}
$$

Due to these varying pressure gradients the flow profile inside the channel will vary from section to section. To minimise these variations, the ratio $l / w$ as defined in Fig. 4.1 should be small. Moreover a constant potential difference between the gate electrode and the bulk fluid at that position should be maintained. This can be achieved with a resistor network as given in Fig. 4.1.

\subsubsection{Resistor network for the gate electrodes}

Let $N$ gate electrodes are connected through $(N-1)$ resistors $R_{\Omega}$, and a variable resistor $R_{\Omega}^{\prime}$ as shown in Fig. 4.1. The electric potential inside the channel with length $L$ at the middle of the $n^{\text {th }}$ electrode at $z_{n}$, due to the field $E_{\infty}=-V_{\infty} / L$ is given by,

$$
\varphi_{2 n}^{\infty}=\frac{z_{n}}{L} V_{\infty}
$$

with

$$
z_{n}=\left(l^{\prime}-l-w / 2\right)+n(l+w), \quad L=\left(l^{\prime}-l+l^{\prime \prime}\right)+N(l+w)
$$

where $w, l, l^{\prime}$ and $l^{\prime \prime}$ are defined in Fig. 4.1. The voltage at the the $n^{\text {th }}$ electrode is given by,

$$
V_{n}=\frac{\left(R_{\Omega}^{\prime}-R_{\Omega}\right)+n R_{\Omega}}{\left(R_{\Omega}^{\prime}-R_{\Omega}\right)+N R_{\Omega}} V_{t o t}
$$

To obtain the same relative surface potential $\psi_{2 n}$ at all gate regions, the potential difference $V_{G}=V_{n}-\varphi_{2 n}^{\infty}=U$ should be independent of $n$. 
This is achieved by choosing $R_{\Omega}^{\prime} / R_{\Omega}$ and $V_{\text {tot }} / V_{\infty}$ such that,

$$
\begin{aligned}
\frac{R_{\Omega}^{\prime}}{R_{\Omega}} & =\frac{U}{V_{\infty}}\left(\frac{l^{\prime}-l+l^{\prime \prime}}{l+w}+N\right)+\frac{l^{\prime}+w / 2}{l+w} \\
\frac{V_{t o t}}{V_{\infty}} & =\frac{U}{V_{\infty}}+\frac{l^{\prime}-l-w / 2+N(l+w)}{l^{\prime}-l+l^{\prime \prime}+N(l+w)}
\end{aligned}
$$

where $V_{t o t}$ is the voltage applied to the resistor network. The typical values of $R_{\Omega}^{\prime} / R_{\Omega}$ and $V_{\text {tot }} / V_{\infty}$ are 100 and 3 .

\subsection{Experiments}

\subsubsection{Channel fabrication}

The channel with chemical modification (channel A) of surface charge is made of two flat borosilicate glass slides (Menzel-Glaser) separated by a para film from which a rectangle $L \times W=14 \mathrm{~mm} \times 0.5 \mathrm{~mm}$ was cut out. The fabrication procedure is described in chapter 2 . Before starting the chemical modification procedure, the glass slides were cleaned with detergent, isopropanol, DI water and kept in $100 \mathrm{mM} \mathrm{KOH}$ at about 90 ${ }^{\circ} \mathrm{C}$ for 30 minutes. After washing in DI water, the glass slides were kept in $3 \% \mathrm{HCl}$ for at least one hour and again washed well in DI water before chemical coating. The glass slide forming the bottom wall of the channel was coated with solutions of $10 \%$ w/v Polybrene (PB), $3 \%$ w/v dextran sulfate (DS), and again $10 \% \mathrm{w} / \mathrm{v}$ PB by subsequently dipping it in the solutions for 10 minutes. After the first PB and DS coating the glass slide was rinsed in DI water for 1 minute. In addition, after the final PB coating, a thorough rinsing was done in DI water at least 3 minutes to remove excess $\mathrm{PB}$ remaining on them. This last step is very important otherwise the excess PB can dissolve into the electrolyte inside the channel and get adsorbed to the other walls and decreases or destroys the shear rate. The height of this channel (A) was $30 \mu \mathrm{m}$. The layout is given in Fig. 2.2B.

In channel $\mathrm{B}$, the upper wall is embedded with gate electrodes. The tungsten gate electrodes were made following a procedure described in 
chapter 2. Each of the five electrodes was $600 \mu \mathrm{m}$ in width and was separated by $60 \mu \mathrm{m}$. This substrate with electrodes was coated with $\mathrm{SiO}_{2} / \mathrm{Si}_{3} \mathrm{~N}_{4} / \mathrm{SiO}_{2}$ trilayer (ONO layer) with total thickness of $750 \pm 50$ $\mathrm{nm}$ to form the insulation layer. The substrate was then annealed at 400 ${ }^{\circ} \mathrm{C}$ for 24 hours. A microchannel of height $20 \mu \mathrm{m}$, width $200 \mu \mathrm{m}$ and length $6 \mathrm{~mm}$ was made of PDMS using soft lithography. The structure of the channel is shown in Fig. 2.1C.

\subsubsection{Measurements}

As the electrolyte solution, we used pH 3 buffer (Fluka, cat no. 82565) dissolved in DI water leading to a $\mathrm{pH}$ 3.6. The conductivity was $120 \mu$ $\mathrm{S} / \mathrm{cm}$ with $1.7 \mathrm{mM}$ concentration of $\mathrm{NaCl}$. We put polystyrene green fluorescent beads of $0.43 \mu \mathrm{m}$ as tracer particles in the electrolyte with volume fraction about $0.005 \%$. The channel and reservoirs were filled with the solution to the same height such that no pressure drop exists along the channel. The flow was recorded using a Nikon Eclipse inverted microscope with $60 \mathrm{X}$ objective and a fast camera (Photron fastcam Ultima 512 ) at 60 or 125 frames per second depending on the strength of the applied electric field to drive EOF. At higher voltages particles move faster so a higher frame rate is required. Recording EOF at strong fields was limited by the low intensity of the images at higher frame rates. While the camera was recording, the channel was scanned along the height by shifting the objective focal plane at $2 \mu \mathrm{m} / \mathrm{s}$ using a piezo element (PI, E-662 LVPZT Position Servo Controller).

In channel $\mathrm{A}$, the flow was recorded, varying driving electric field, $E_{\infty}$ from 3.5 to $10.7 \mathrm{~V} / \mathrm{mm}$. In channel $\mathrm{B}$, driving electric field was varied from 2.5 to $12.5 \mathrm{~V} / \mathrm{mm}$ while applying a gate voltage, $V_{G}$ of $500 \mathrm{~V}$. Also the gate voltage was varied from 0 to $500 \mathrm{~V}$ while the driving field was kept fixed at $5 \mathrm{~V} / \mathrm{mm}$. For each applied driving electric field and gate voltage $V_{G}$ combination, the variable resistance $R_{\Omega}^{\prime}$ was adjusted such that a constant potential difference exists between every gate electrodes and the bulk of the fluid at the position of the electrode.

The recorded video of the flow at each focal plane was analysed with 
imageJ using a particle tracking algorithm [74]. The trajectory data generated from ImageJ were then analysed with home made matlab code to extract the velocity of each particle detected. These velocities were then averaged. This velocity is $E_{\infty}$ times the sum of EO mobility and electrophoretic mobility of the particles. The EO mobility can be calculated knowing the electrophoretic mobility which is measured separately as $0.046 \times 10^{-6} \mathrm{~m}^{2} /$ Vs using ZetaSizer (Malvern).

\subsection{Results and discussion}

\subsubsection{Chemically modified zeta potential}

The flow profiles measured for PB modified channel is given in Fig. 4.2A. The curves show a linear change in the EOF velocity along the channel height. The quantity $m(y)=u_{E O F} / E_{\infty}$ is plotted in Fig. 4.2B. The values of $m(-h / 2)$ and $m(h / 2)$ give the EO mobility near the PB coated and the bare glass as $+0.036 \times 10^{-6} \mathrm{~m}^{2} / \mathrm{Vs}$ and $+0.058 \times 10^{-6} \mathrm{~m}^{2} / \mathrm{Vs}$ respectively. The inset of Fig. 4.2B shows the shear rate $\dot{\gamma}$ versus the field strength $E_{\infty}$. The slope of this curve is $(0.75 \pm 0.01) \times 10^{-3} \mathrm{~m} / \mathrm{Vs}$. This slope must be equal to the magnitude of the slope of $m(y)$ versus $y$ (Fig. 4.2B) which is $(0.70 \pm 0.05) \times 10^{-3} \mathrm{~m} / \mathrm{Vs}$. Since for a stable shear flow the chemical content on the glass is limited, we assume that this is the typical shear rate per field that can be achieved with chemical modification for a given channel with height $h$.

The surface charge of PB coated glass was not positive as anticipated but slightly negative. This is because the PB coated glass slides were rinsed in DI water such that most of the PB washes away. When the coated glass slides are not rinsed excessively, the polymers from one wall dissolve in the electrolyte and adsorb onto the other wall. The shear flow is then destroyed within few minutes. By keeping only low PB content on the coated glass slide, we were able to maintain a stable shear flow for more than ten measuring hours. When bare silica capillaries are fully coated with PB by successive flushing with PB and DS solutions, the wall 

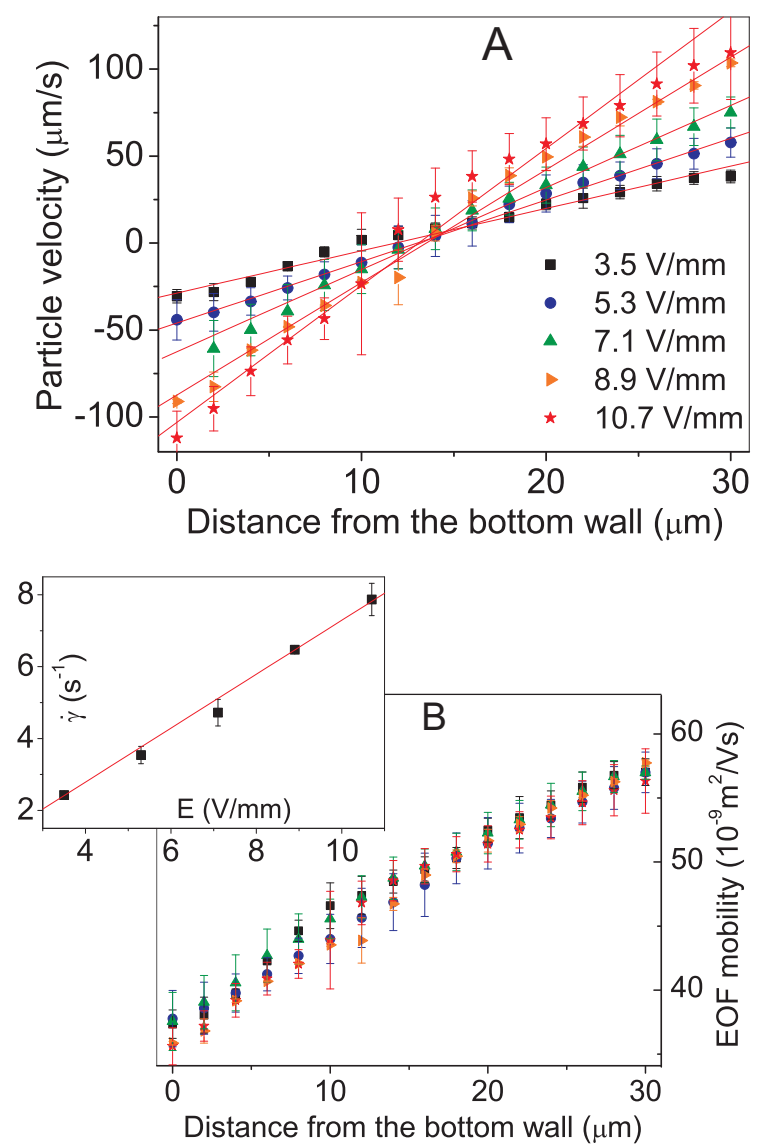

Figure 4.2: (A) The flow profiles measured for channel A at different values of $E_{\infty}$. (B) The EOF mobility, $m(y)=u_{E O F} / E_{\infty}$. The inset shows the shear rate versus $E_{\infty}$.

surface charge was measured as $+18.0 \pm 4 \mathrm{mC} / \mathrm{m}^{2}$ by solution displacement method [107]. The surface charge of the PB coated capillaries is independent of the $\mathrm{pH}[99,102]$. 


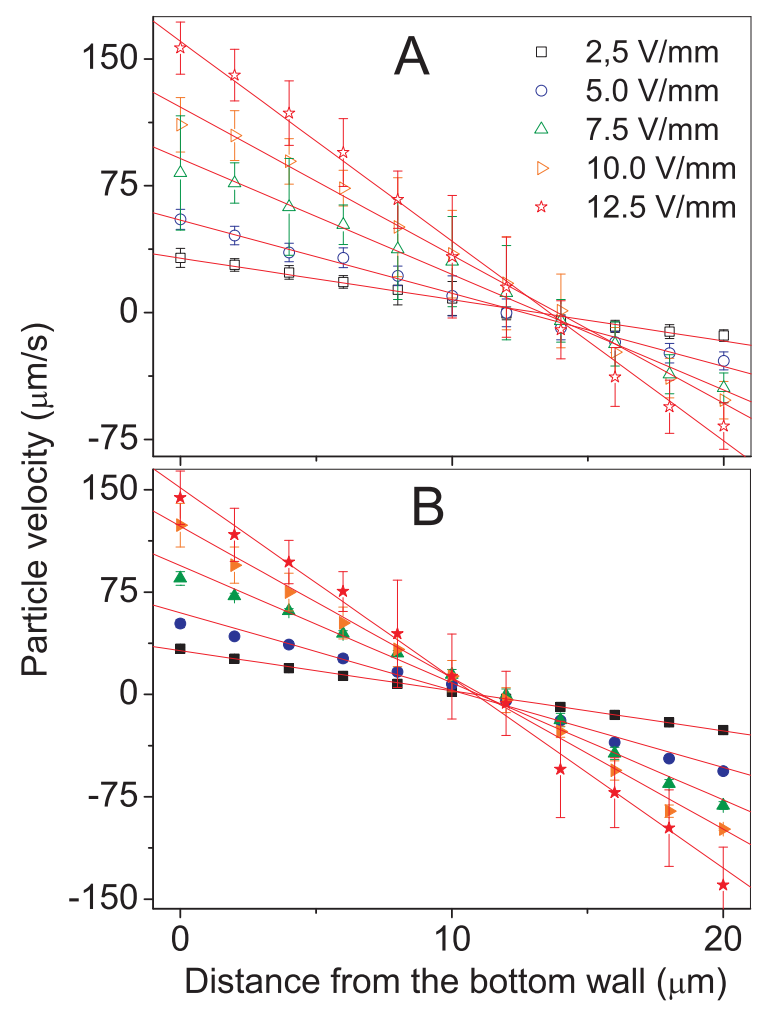

Figure 4.3: The flow profiles measured for the channel B at different values of $E_{\infty}$. The profiles are fitted linear. (A) $V_{G}=0 \mathrm{~V}$. (B) $V_{G}=500 \mathrm{~V}$.

\subsubsection{Electrically modified zeta potential}

With chemical modification, the glass slides cannot be coated to obtain certain desired surface charge on it. But modulating the zeta potential by gate voltage, enables to control the shear rate not only by changing the driving voltage but also with the gate voltage. And also larger shear rates can be achieved without using chemicals in the channel.

The flow profiles measured with channel $\mathrm{B}$, at different electric fields ranging from 2.5 to $12.5 \mathrm{~V} / \mathrm{mm}$ keeping the gate voltage at $500 \mathrm{~V}$, are shown in Fig. 4.3A. The function $m(y)$ is with and without gate voltage is 
shown in Fig. 4.4. Near the PDMS wall at the bottom of the channel, EOF mobility is $(+0.058 \pm 0.007) \times 10^{-6} \mathrm{~m}^{2} / \mathrm{Vs}$. The corresponding surface charge and zeta potential are $8 \pm 1 \mathrm{mC} / \mathrm{m}^{2}$ and $-82 \pm 10 \mathrm{mV}$. Near the upper wall of ONO layer, the mobility is $(+0.039 \pm 0.005) \times 10^{-6} \mathrm{~m}^{2} / \mathrm{Vs}$. The corresponding surface charge and zeta potential are $5 \pm 0.5 \mathrm{mC} / \mathrm{m}^{2}$ and $-55 \pm 7 \mathrm{mV}$. Due to the low surface charge of PECVD deposited $\mathrm{SiO}_{2}$ layer compared to that of PDMS, a linear shear flow exists in the channel. The corresponding shear rates are shown in the Fig. 4.5. At zero gate voltage, shear rate per field is $(0.88 \pm 0.05) \times 10^{-3} \mathrm{~m} / \mathrm{Vs}$. The magnitude of the slope of $m(y)$ versus $y$ (Fig. 4.4) is $(0.90 \pm 0.07) \times 10^{-3}$ $\mathrm{m} / \mathrm{Vs}$.

When gate electrode is kept at $500 \mathrm{~V}$, the mobility measured near the upper wall is $(+0.035 \pm 0.004) \times 10^{-6} \mathrm{~m}^{2} / \mathrm{Vs}$. This corresponds to an average zeta potential of the wall. The actual zeta potential can be calculated as $-43 \pm 5 \mathrm{mV}$ by using Eq.(4.2.1). Thus the gate voltage made a change in zeta potential by $13 \pm 12 \mathrm{mV}$. As shown in Fig. 4.5, when the gate voltage is applied, the shear rates are increased by about $42 \%$ and the shear rate per field is $(1.3 \pm 0.05) \times 10^{-3} \mathrm{~m} / \mathrm{Vs}$. It must be same as the magnitude of the slope of $m(y)$ versus $y$ (Fig. 4.4) which is $(1.20 \pm 0.08) \times 10^{-3}$ $\mathrm{m} / \mathrm{Vs}$. The inset of Fig. 4.5 shows shear rate with gate voltage at a fixed driving field of $5 \mathrm{~V} / \mathrm{mm}$. The shear rate seems to saturate at high gate voltage. This is because the change in zeta potential saturates at high gate voltage due to the nonlinear behaviour of the double layer capacitance in Eq.(4.14). The shear rate can also be decreased by applying a negative gate voltage.

We used water as the working fluid and since its viscosity is known, we calculate the zeta potential of the wall from the measured shear rate and compare with the value that obtained from a separate measurement by solution displacement method [107]. From the shear rate (in channel A) and from the separate measurement, we obtained the zeta potentials of the bare glass wall as $80 \pm 2$ and $80 \pm 3 \mathrm{mV}$ respectively. This shows that, for a working fluid of unknown viscosity (for example an aqueous suspension of colloids), by knowing the zeta potentials, it's viscous properties can be 


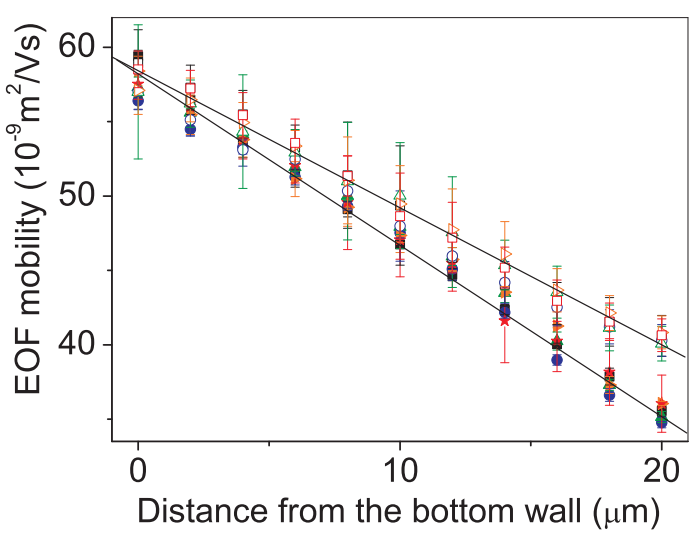

Figure 4.4: The EOF mobility $m(y)=u_{E O F} / E_{\infty}$ for $V_{G}=0 \mathrm{~V}$ (open symbols) and $V_{G}=500 \mathrm{~V}$ (filled symbols). The lines are a guide to the eye.

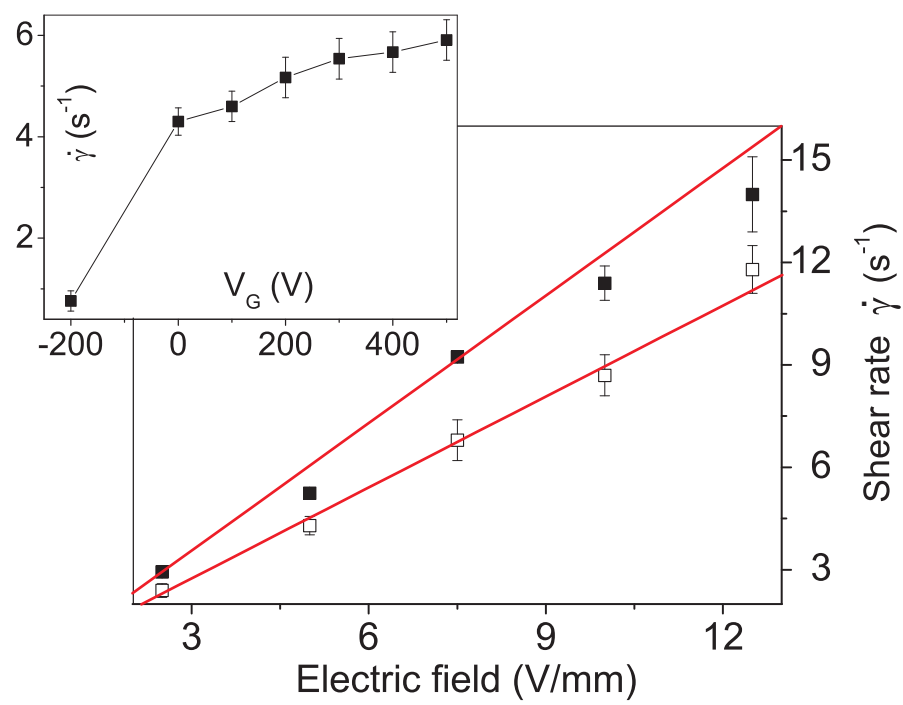

Figure 4.5: The shear rates with gate voltage of 0 and $500 \mathrm{~V}$ versus $E_{\infty}$. The red line is the linear fit. The inset shows variation of shear rate with gate voltage at fixed $E_{\infty}=5 \mathrm{~V} / \mathrm{mm}$. 
probed.

As discussed in section 2.1, there are local pressure gradients building up in the channel due to the inhomogeneity in zeta potential on the upper wall. Using Eq. (4.18), we calculated that these pressure gradients contribute $9 \%$ to the average EOF velocity. This calculation assumes very sharp interface between each regions which is not the case for the actual flow. Therefore the linear flow profiles those we measured are justified. The ratio $l / w$ of the channel is 0.1 . By decreasing this ratio further the pressure contribution can be even minimized. The inhomogeneity in zeta potential created by the finite width of a gate electrode is less than $1.4 \%$ in our measurements. However this can be improved by further decreasing the width of the electrodes while keeping $l / w \ll 1$. Using several electrodes on the channel wall is therefore necessary.

The change in zeta potential by the applied voltage on the gate electrodes was much lower (by a factor of 6) than that expected theoretically. We attribute this to the high buffer capacity of PECVD deposited $\mathrm{SiO}_{2}$ dielectric layer which resulted in very low efficiency of the gate voltage (see also appendix A). The most of the applied gate voltage is buffered by the dielectric layer by releasing protons. Hence the zeta potential is not much changed from the zero bias situation. The native surface charge of such layers is slightly different in various samples and is always less than that of glass or PDMS. This is because the $\mathrm{SiO}_{2}$ layers are grown from the reaction of $\mathrm{SiH}_{4}$ and $\mathrm{N}_{2} \mathrm{O}$ and the prepared layers may have doped with some amino groups at very small concentration. We found that the surface charge of such PECVD deposited layers is not stable over time compared to that of bare glass. Therefore it is advised to flush the channel with $100 \mathrm{mM} \mathrm{NaOH}$ or $\mathrm{KOH}$ in order to activate all surface groups on the wall. Also, fill the channel with the electrolyte few minutes before starting the measurements such that the surface and the liquid are in well equilibrium.

Gate actuation of EOF requires good dielectric layers over the electrodes. Mostly PECVD grown very thin layers have low breakdown voltage in contact with aqueous liquids. This is due to the small pinholes of 
PECVD grown layers. The same layers show high breakdown voltage in solid-solid electrode contact or even with ionic liquids. This issue may be solved by using low pressure chemical vapour deposition (LPCVD) to prepare the dielectric layers. Currently we make electrodes on the silicon wafer by doping with boron and an LPCVD silicon nitride layer is grown as insulation.

\subsection{Conclusion}

We have shown that linear electroosmotic shear flow can be created by using chemical modification of surface charge. The adsorption of chemicals by the opposite bare wall can destroy the shear flow. Therefore this technique is limited by the amount of chemical coating that can present on one of the channel walls. This can be overcome by using gate voltage to change the zeta potential of the channel wall. The $\mathrm{SiO}_{2}$ dielectric layer deposited on the gate electrodes by using PECVD technique, has very low negative surface charge compared to glass or PDMS. This property also can be used to create shear flow. By modulating the surface charge of this $\mathrm{SiO}_{2}$ layer with gate voltage, shear rate is increased by $42 \%$. By increasing the electric field $E_{\infty}$ along the axis of the channel, higher shear rates can be produced. Instead, this can be also achieved by increasing the efficiency of the gate voltage, by using other kind of dielectric materials such as $\mathrm{Al}_{2} \mathrm{O}_{3}$ or LPCVD deposited silicon nitride layer. 



\section{Chapter 5}

\section{Controlling flow patterns in oscillating sessile drops}

Part I: We study flow patterns inside sessile drops, which oscillate under the influence of an AC voltage applied in electrowetting configuration. We show that the strength of the flow field correlates with the eigen modes of the drop in the polar direction and - most importantly - that the azimuthal symmetry of the resulting flow fields can be broken by introducing pinning sites along the contact line of the drop. We anticipate that the controlled introduction of azimuthal vortices increases the mixing efficiency inside the droplet.

Part II: When the frequency of the AC applied voltage is gradually increased to a few tens of kilo Hertz the azimuthal flow vortices move towards the contact line near the pinning site. These moving flow vortices sweep micro/nano particles or even DNA dispersed in the droplet towards the pinning sites. As a result, the particle concentration increases locally inside the drop by a factor of about ten. 


\section{Part I: Controlling flow patterns}

\subsection{Introduction}

Discrete microfluidic drops are frequently used as reaction containers in digital microfluidic systems. In order to guarantee fast and well-controlled chemical reaction kinetics efficient mixing schemes for the fluid inside the drops are needed. In the case of sessile drops of flat surfaces internal flow patterns can be generated by shaking the drops at frequencies comparable to the eigen frequency of the drop. Such drop oscillations can be driven particularly efficiently by applying an alternating (AC) voltage to a drop in electrowetting (EW) [19] configuration. The EW-induced periodic modulation of the contact angles induces a largely periodic oscillatory flow pattern inside the drop. Yet, it has been shown that a net drift flow giving rise to mixing is usually superimposed onto this periodic flow. This net flow is caused by a symmetry breaking of the drop shape between the spreading and the receding phase in each oscillation cycle. The physical mechanism causing the symmetry breaking is not fully clear. Differences in the orientation of the net flow in the polar direction between experiments in ambient air [59] and in ambient oil [58, 61] suggest that different mechanisms such as inertial effects and contact line (de)pinning dynamics may dominate, depending on the conditions of the experiments. The symmetry in the azimuthal direction equally seems to depend on the details of the experimental conditions. While axisymmetric radial flow patterns were observed in some experiments [58, 61, 108], vortices in the azimuthal plane were reported in others [60].

In this chapter, we analyse the strength and the symmetry of the net internal flow patterns in EW-driven oscillating drops in ambient air. We demonstrate that the strength (average speed) of the flow correlates with the eigen modes of the drops in the polar direction that can be addressed one-by-one by sweeping the drive frequency. On homogeneous surfaces, the symmetry of these flow patterns in the azimuthal direction is ill-defined and typically varies between twofold and fourfold from drop to drop in an uncontrolled manner. By introducing artificial pinning sites along the 


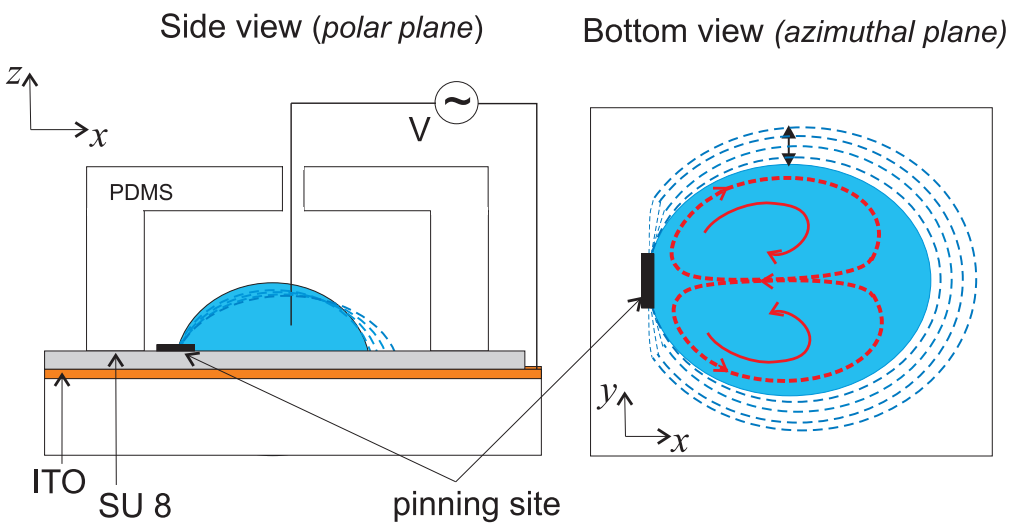

Figure 5.1: Side (A) and bottom (B) view of the experimental set up (drop not to scale, typical diameter is $1 \mathrm{~mm}$ ). The blue dotted lines indicate the motion of the contact line, the red dashed lines in (B) the internal drift flow.

contact line of the drop, this randomness can be overcome and a welldefined number of vortices (twice the number of pinning sites) can be imposed. While the number of pinning sites determines the number vortices independent of the drive frequency, the lateral dimensions of the vortices are found to decrease with increasing frequency.

\subsection{Experiments}

The experiments were performed in a basic EW set-up, as depicted in Fig. 5.1. It consists of a glass substrate coated with Indium-Tin-Oxide (ITO). A $5 \mu \mathrm{m}$ thick dielectric film of SU8 has been coated on the ITO layer. SU8 has an equilibrium contact angle of $85^{\circ}$ for water in air. Additionally, on part of these substrates artificial pinning sites were created by coating small gold stripes ( $200 \mu \mathrm{m}$ in width) or patches of silver paste on the SU8 film. Drops of $10 \mathrm{mM} \mathrm{KCl}$ salt solution in deionized water with a typical volume of $1 \mu \mathrm{L}$ were deposited on the substrate. These drops contain approximately 500 fluorescent tracer particles $/ \mu \mathrm{L}$ with a diameter of 
$5 \mu \mathrm{m}$. The drop is covered by a small Polydimethylsiloxane (PDMS) enclosure which creates a saturated atmosphere of water vapor that prevents evaporation during the experiments. An rms voltage of $190 \mathrm{~V}$ (resulting in an average contact angle of about $50^{\circ}$ ) was applied though a platinum wire inserted in the droplet through a small hole in the PDMS cover. The droplet and its tracer particles are observed from below using an inverted microscope equipped with a CCD camera. The motion of the particles is recorded in a plane about $50 \mu \mathrm{m}$ above the substrate.

\subsection{Results and discussion}

First we discuss experiments using a bare SU8 surface (without artificial pinning sites). By applying an $\mathrm{AC}$ voltage to the droplet we observe not only internal flows in the vertical symmetry plane as already reported in $[59,58,61]$, but also a flow pattern in the horizontal plane, consisting of 2 or 4 vortices. We recorded these flow patterns at $125 \mathrm{fr} / \mathrm{s}$, while sweeping the AC frequency from $100 \mathrm{~Hz}$ to $2 \mathrm{kHz}$ at a rate of $16 \mathrm{~Hz} / \mathrm{s}$, for droplets with a volume ranging from 0.6 to $5 \mu \mathrm{L}$. The trajectories of the tracer particles have been extracted from the recorded movies [74]. From these trajectories the particle velocities were determined using a home made Matlab code. By averaging the individual particle speeds, using their trajectory length as a weight factor (see chapter 2), the characteristic flow speed at a given frequency was calculated.

In Fig. 5.2 this characteristic flow speed has been plotted versus the applied frequency for a $1 \mu \mathrm{L}$ droplet. It clearly shows distinct peaks at certain frequencies. Droplets with different volume show similar behaviour with peak velocities ranging from 0.2 to $2.5 \mathrm{~mm} / \mathrm{s}$. The height of the peaks varies slightly from droplet to droplet, depending upon the local surface conditions of the substrate near the droplet. In our analysis we focus on the frequency behaviour. For six different drop volumes we compare the frequencies $f_{P}$, corresponding to the peaks in the characteristic flow speed, with the resonance frequencies $f_{n}$, for surface oscillations of 


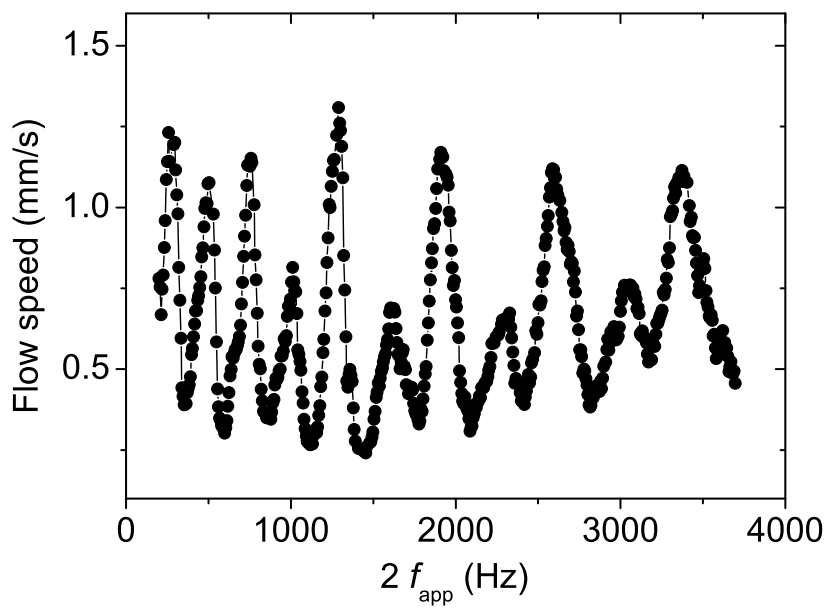

Figure 5.2: Characteristic flow speed in the azimuthal plane versus driving frequency for a $1 \mu \mathrm{L}$ droplet. The contact line oscillates with twice the applied frequency, $f_{a p p}$ (see chapter 1). The curve shows distinct peaks which correlate with the calculated frequencies $f_{n}$.

spherical drops as given by Rayleigh's formula [109],

$$
f_{n}=\sqrt{n(n-1)(n+2) \gamma / 4 \pi^{2} \rho R^{3}}
$$

where $\gamma\left(0.073 \mathrm{Nm}^{-1}\right)$ is the surface tension and $\rho\left(10^{3} \mathrm{~kg} / \mathrm{m}^{3}\right)$ is the density of the liquid. To calculate $f_{n}$ we estimate the drop radius $R$ from the average contact line radius, $R_{0}$ and the contact angle, $\theta$ as $R=R_{0} / \sin \theta$. In Fig. 5.3, both $f_{P}$ and $f_{n}$ have been plotted logarithmically as a function of $R^{3}$ for even modes with $n$ ranging from 4 to 18. Only even modes are expected due to the symmetry in the polar angle plane assuming a free slipping contact line. The $n=2$ mode is in principle also accessible but at a frequency below the applied range, so this mode is not observed in our experiments. A power law fit (not shown) to the $f_{P}$ versus $R^{3}$ curves corresponding to each mode reveals exponents ranging from $-0.47 \pm 0.06$ to $-0.6 \pm 0.1$ which is in agreement with the expected value of $-1 / 2$, i.e. 


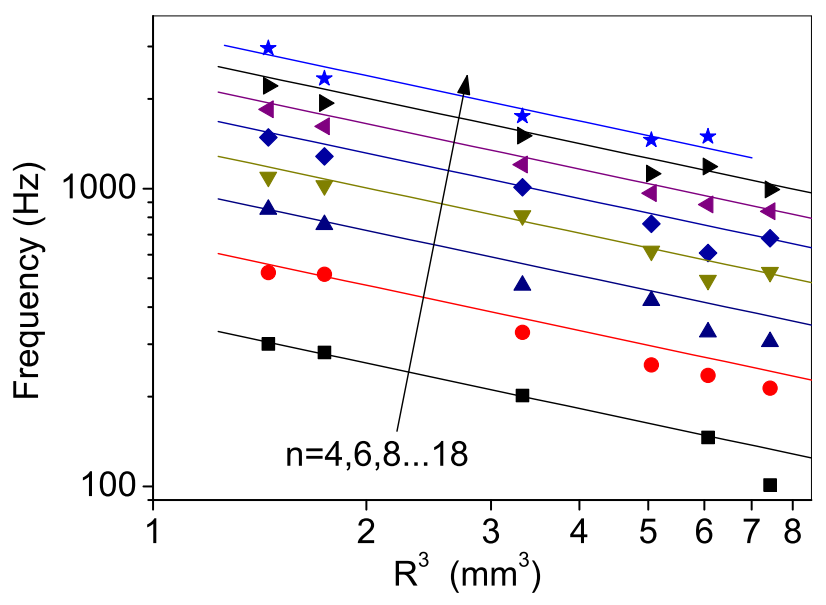

Figure 5.3: Frequencies $f_{P}$ corresponding to the peaks in the azimuthal flow speed (symbols) versus drop volume. Lines: resonance frequencies $f_{n}$ (for oscillations in the polar plane) calculated from Rayleigh's formula.

the slope of the lines in Fig. 5.3. An error of about $4 \%$ is expected in the calculated value of $f_{n}$ which originates from the uncertainty of $2^{\circ}$ in the contact angle determination. Although the overall mismatch is less than $25 \%$, the values of $f_{P}$ lie systematically below $f_{n}$ but obey the same scaling behaviour. We attribute this deviation in resonance frequencies to the sessile drop shape [136] and the stick slip motion of the contact line that breaks the symmetry at the central plane [111]. Also the finite viscosity of the droplet will lower the the resonance frequencies slightly. For the large drops $(4$ and $5 \mu L$ ) resonance behaviour may have effected from gravity also. Overall, these measurements show a strong correlation in the frequency dependence between the observed flow patterns in the azimuthal plane and the expected surface oscillations of the droplet in the polar direction.

To gain control over the azimuthal vortex patterns, we introduced artificial pinning sites along the contact line of the drop and studied the 

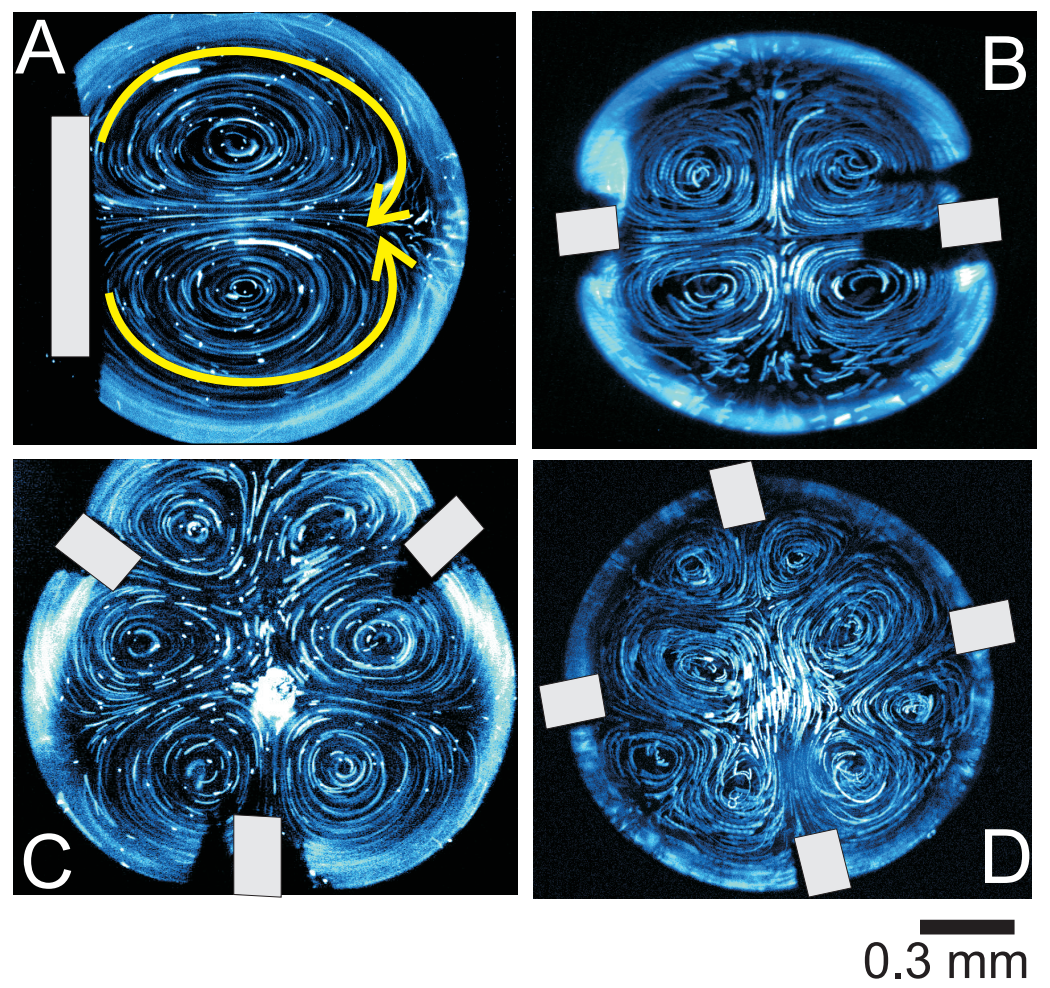

Figure 5.4: Bottom view of the flow patterns. The white bars represent the positions of the pinning sites.

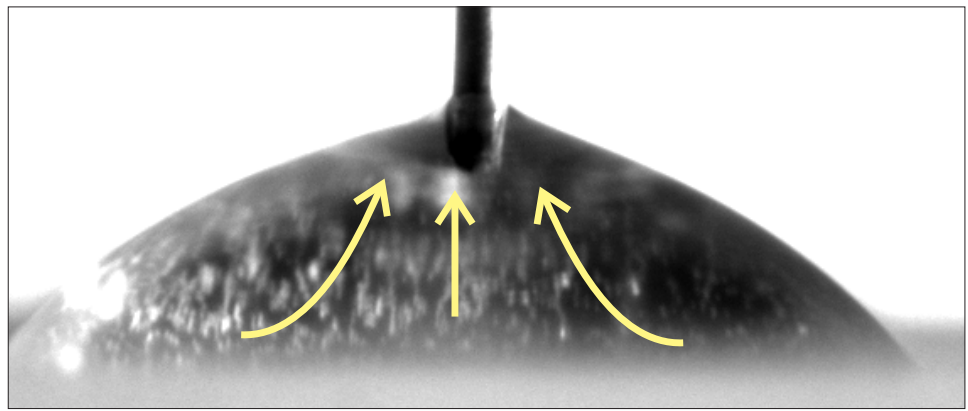

Figure 5.5: The side view of the drop shows a weak upward flow. 

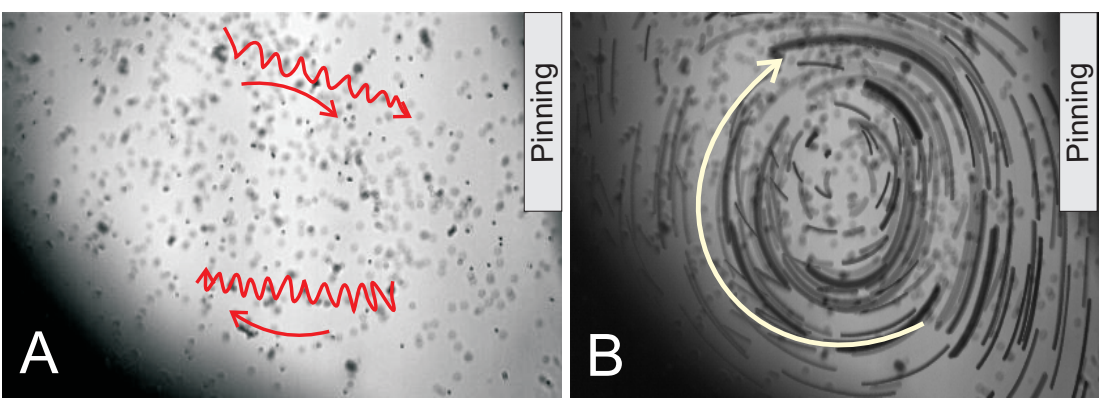

Figure 5.6: A) The bottom view image of the drop shows particles show radial oscillations and a drift motion in the azimuthal plane as illustrated in red (guide to eyes). B) Superimposing all the frames of the movie shows the trajectories of the particles.

vortex behaviour as a function of applied frequency. Fig. 5.4 shows the flow inside droplets driven with a frequency of $1 \mathrm{kHz}$ for 1 to 4 pinning sites. For each pinning site, two vortices occur, independent of the applied frequency. The direction of the flow in the vortex is always directed away from the pinning site along the edge of droplet and towards the unpinned region as indicated by the arrows in Fig. 5.4A. With increasing number of pinning sites, the space available to a single vortex decreases and the pattern becomes more irregular as is most clearly observed in Fig. 5.4D. A side view of the drops shows that apart from the in plane vortices also a weak upward flow at the center of the droplet occurs (Fig. 5.5). This is in agreement with observations reported by Ko et al. [59].

For a better insight in the origin of this flow we investigated the short time dynamics of the tracer particles in a drop with one artificial pinning site, using a high speed camera at $10^{4} \mathrm{fr} / \mathrm{s}$. We resolved the expected oscillatory motion of the particles in the radial direction with a frequency corresponding to twice the driving frequency, together with a drift motion in the horizontal plane (see Fig. 5.6). This drift is determined from the particle displacements during a full oscillation cycle. The time averaged drift trajectories result in the vortices as shown in Fig. 5.4.

We suppose that the azimuthal flow originates from the broken sym- 


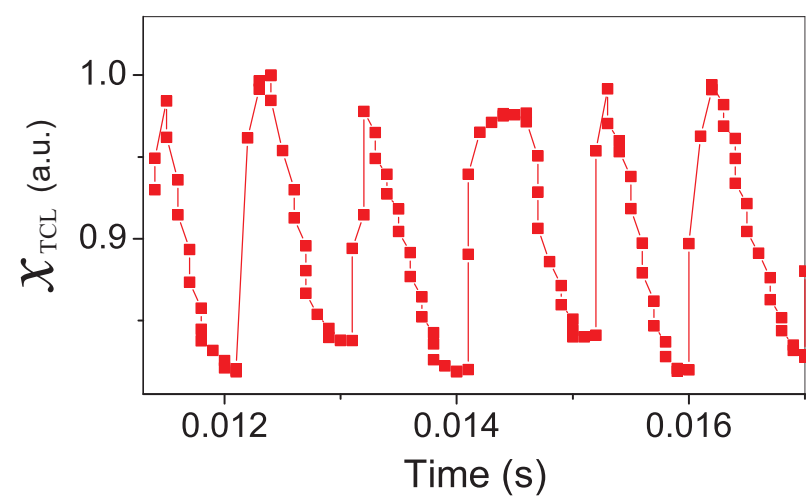

Figure 5.7: The position of the contact line versus time when a sinusoidal voltage with frequency $500 \mathrm{~Hz}$ is applied. The contact line advances very quickly but recedes slowly.

metry of the stress oscillations at the three phase contact line. The suppression of the stress oscillations at the pinning sites results locally in a different amplitude of the contact line motion (see Fig. 5.1B). Consequently, an aperiodic drift motion superimposes the periodic oscillations of the liquid. This drift forms the flow patterns over many oscillation cycles. The nonlinear effect causing this drift is not well understood. Stick-slip like oscillations (see Fig. 5.7) of the contact line caused by the roughness of the surface may bring forth contributions of inertia.

\subsection{Conclusion}

In conclusion, our experiments show that $\mathrm{AC} \mathrm{EW}$ drives interface oscillations and internal drift flow patterns, not only in the vertical plane but also in the azimuthal plane, parallel to the substrate. Using artificial pinning sites, one can control the vortex pattern in the azimuthal plane. These vortices will enhance the mixing efficiency inside the drop in azimuthal direction. The frequency dependence of the flow strength in these vortices is described by the eigen frequencies of the corresponding droplet surface oscillations. The described internal flow and anticipated enhanced mix- 
ing efficiency have potential applications, in stirring and mixing on the $\mu \mathrm{L}$ scale or suppressing the settling of (micro) particles in drying drops, e.g. the coffee stain effect [64]. This will be useful in optimizing existing applications.

\section{Part II: Sample pre-concentration}

\subsection{Introduction}

Microfluidic techniques have the advantage of consuming only very little amount of sample for bio/chemical analyses. When the sample contains analyte at very low concentration, it is essential to meliorate the sensitivity of such techniques. Improving the sensitivity of the detection system is complicated and expensive. Alternatively, a sample pre-concentration can be performed. Different methods for sample pre-concentration are reviewed by Chun-Che Lin et al [112]. They include techniques based on surface binding, porous membranes, nano channels, solvent extraction or velocity gradients. Most of the above mentioned techniques are applied in continuous flow microfluidics.

Recently discrete droplets are used as compartments for cells or chemical reaction chambers [51]. Also in this case sample pre-concentration would be beneficial. Solutes within volatile drops in ambient air can be concentrated by evaporation of the solvent. Similarly, solutes and particles entrapped in aqueous micro drops in ambient oil phase can be concentrated by letting the water molecules from the drop dissolve into the organic phase [113]. Cho et al [114] proposed another method by concentrating the particles electrophoretically on one side in the drop and subsequently splitting the drop by electroweting. This produces daughter drops having higher particle concentration.

Most of the methods described in the literature require strong electric fields and the conductivity or the $\mathrm{pH}$ of the fluid must be tuned for optimal performance. In biological applications, these invasive methods are not 
favourable. To the best of our knowledge there are no non-invasive methods reported in literature to pre-concentrate the solutes within a sessile droplet without moving or splitting it.

In this part, we demonstrate a novel method for enhancing the local concentration of colloidal particles or bio-molecules in a sessile micro droplet using AC electrowetting. Not only mixing, but an inverse mixing i.e. concentration enhancement, can also be achieved by manipulating the flow using artificial pinning sites at the contact line of the drop. This is accomplished by sweeping the frequency of the applied voltage from low $(\sim 1 \mathrm{kHz})$ to high $(\sim 50 \mathrm{kHz})$ value. As seen from part I, a single pinning site creates two flow vortices inside the drop. By gradually increasing the frequency, these flow vortices can be shifted towards the pinning site at the contact line. The shifting vortices collect particles or bio-biomolecules and accumulate them near the pinning site.

\subsection{Experiments}

The experimental setup is similar Fig. 5.1A. Drops of deionised water containing $10 \mathrm{mM} \mathrm{KCl}$ and having volume ranging between 0.4 to $1 \mu \mathrm{L}$ are brought on the substrate such that the contact line partially touches the pinning site. The drops also contained colloidal particles of diameter ranging from $20 \mathrm{~nm}$ to $5 \mu \mathrm{m}$ with volume fraction of $0.05 \%$ or DNA with a concentration of $2.5 \mathrm{ng} / \mu \mathrm{L}$ prepared by diluting fluorescently labelled (SYTO 24) stock solution of cDNA with deionized water containing 10 $\mathrm{mM} \mathrm{KCl}$.

A voltage of $150 \mathrm{~V}$ is applied between the ITO layer and the metallic pinning stripe as shown in Fig. 5.8. The frequency of the applied voltage is swept from $1 \mathrm{kHz}$ to typically $50 \mathrm{kHz}$ with a typical rate of $1 \mathrm{kHz} / \mathrm{s}$. A confocal microscope is used to observe the particles at a plane about 100 $\mu \mathrm{m}$ above the substrate. An exposure time of $0.5 \mathrm{~s}$ is used to image the colloidal particles and $3 \mathrm{~s}$ for imaging DNA. 


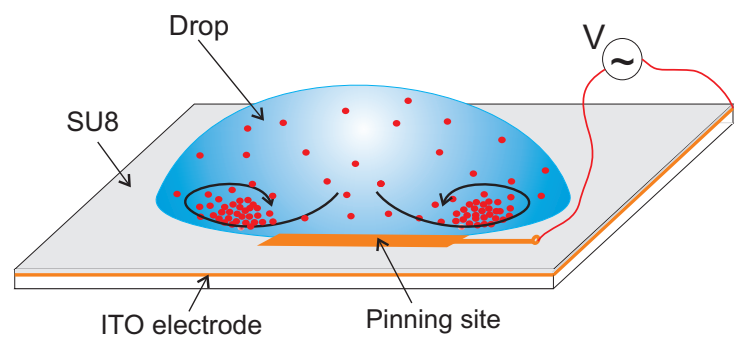

Figure 5.8: The experimental setup (see also Fig. 5.1).

\subsection{Results and discussion}

The bottom view images of the drops before and after the frequency sweep are shown in Fig. 5.9. The drops contain $20 \mathrm{~nm}, 500 \mathrm{~nm}, 5 \mu \mathrm{m}$ particles or DNA. The left panel shows the drops in the beginning of the frequency sweep $(1 \mathrm{kHz})$ and the right panel shows those at the end $(50 \mathrm{kHz})$. For $5 \mu \mathrm{m}$ particles two large flow vortices are visible at $1 \mathrm{kHz}$ frequency. For the other drops the vortices are not visible due to the small size of the particles. As the frequency increases, the flow vortices get smaller and shift towards the pinning region while collecting the particles along with them. Each flow vortex thus concentrates particles separately near the pinning site. The increased fluorescent intensity at two places near the pinning site indicates increased number of particles at those places. DNA is often accumulated at random positions. We attribute this behaviour of DNA to it's high adsorption on SU8 surface [115]. At the final stage of

the frequency sweeping, the voltage can be turned off. This makes the drop retract to it's initial contact angle. The retracting motion partially spreads the collected particles. Therefore all the images of the drops at the final stage of the frequency sweep are taken while the voltage is on. In most of the optical analyses techniques, the analyte is detected fluorescently. Pre-concentrating them will increase the fluorescent signal as seen from Fig. 5.9. Typically the intensity is enhanced by a factor of ten. For instance, the intensity profiles for the drop with $500 \mathrm{~nm}$ particles 

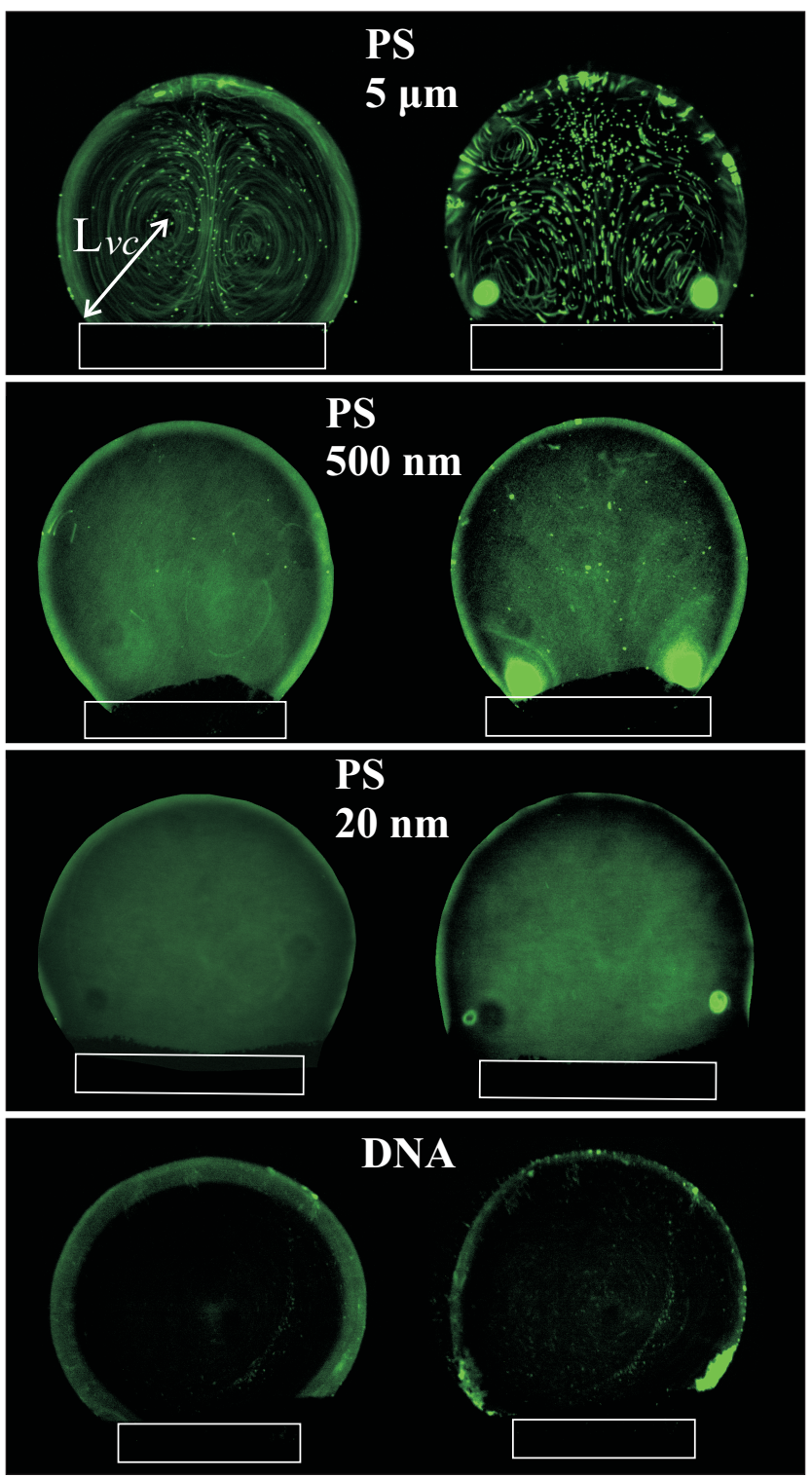

$300 \mu \mathrm{m}$

Figure 5.9: The bottom view images of the drops with colloidal particles of diameter $5 \mu \mathrm{m}, 500 \mathrm{~nm}, 20 \mathrm{~nm}$ or DNA. Left and right panels represent drop before and after the frequency sweep respectively. The bright brim of the drops is due to reflection. The distance between the vortex center and the pinning site is defined as $L_{v c}$. 


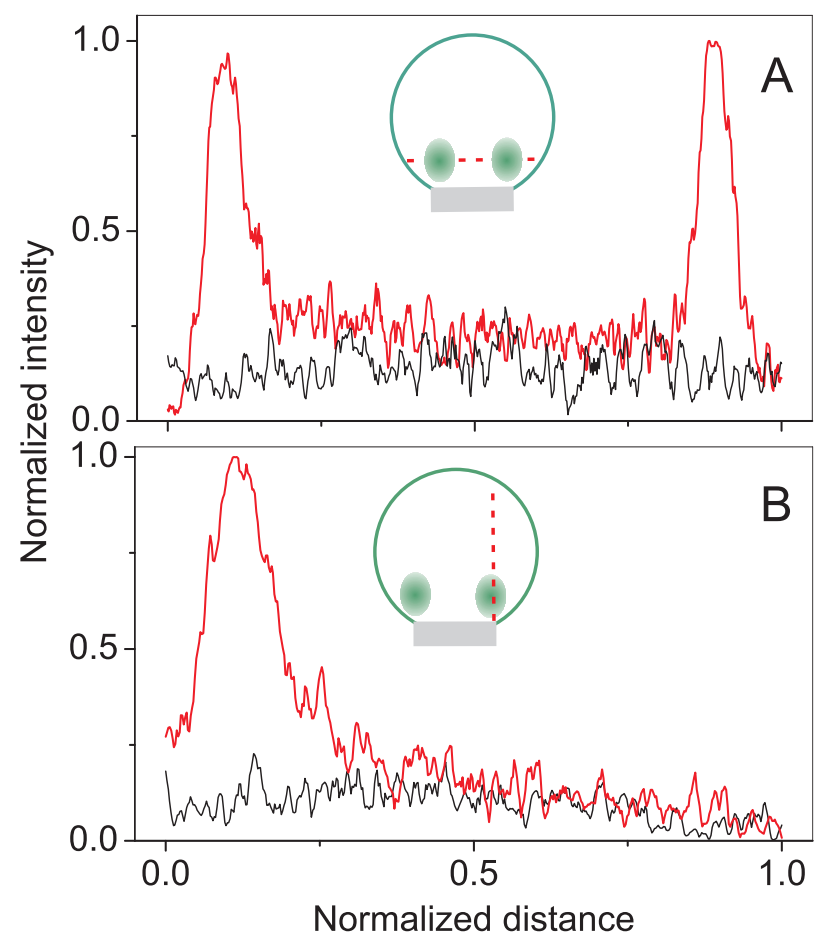

Figure 5.10: The intensity profile for the drop containing $500 \mathrm{~nm}$ particles before (black) and after (red) the frequency sweep. The intensity is normalized with respect to it's maximum value. Distance is normalized with respect to the total profiling length. The inset shows the region where intensity cross section is made.

show an enhancement by a factor of $10 \pm 2$ (Fig. 5.10). The inset is a cartoon of the drop with the dash line indicating the region where the intensity is profiled. Likewise, for the drops with $5 \mu \mathrm{m}, 20 \mathrm{~nm}$ particles and DNA, the intensity was enhanced by a factor of $11 \pm 2,10 \pm 3$ and 17 \pm 2 respectively. The enhancement in intensity depends on the amount of particles collected near the pinning site. When the frequency sweeping rate is very high, the vortices move quickly towards the pinning site. Consequently, only a few number of particles are collected. This is clear from Fig. 5.11 where the intensity enhancement (=final intensity/initial 
intensity) near the pinning site versus the sweeping rate is plotted. At low sweeping rates, i.e. below about $2 \mathrm{kHz} / \mathrm{s}$ the final intensity is maximum and stays constant. A slow sweeping resulted in a relative increase of the final intensity by almost three times.

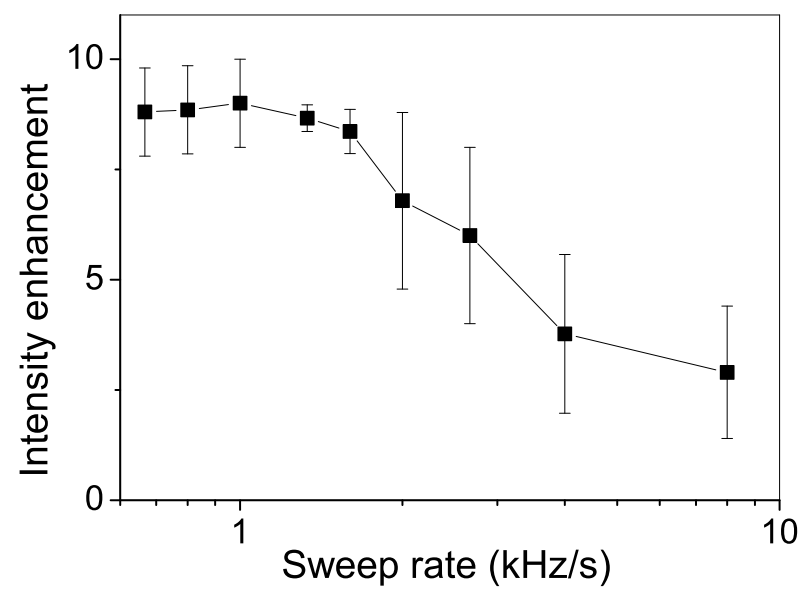

Figure 5.11: The intensity enhancement (= final intensity/initial intensity) near the pinning site versus the sweep rate. The frequency is swept from 1 to $40 \mathrm{kHz}$ at different rates.

Not only the rate of sweeping but the high frequency where the sweeping ends is also a crucial parameter. The final frequency must be chosen such that the vortices reach as close as possible to the contact line. We took the distance between the pinning site and the vortex center as a characteristic measure of the position of a vortex. This distance $L_{v c}$ as a function of the frequency for drops with three different volumes $(0.4,0.7$ and $1 \mu L)$ are shown in Fig. 5.12. When the applied frequency $f_{a p p}$ is above about $25 \mathrm{kHz}$, the vortices reach near the contact line and do not shift any further. Moreover, it is found that below an onset frequency (marked by the arrow), the vortices do not shift. This onset frequency is relatively larger for smaller drops. 


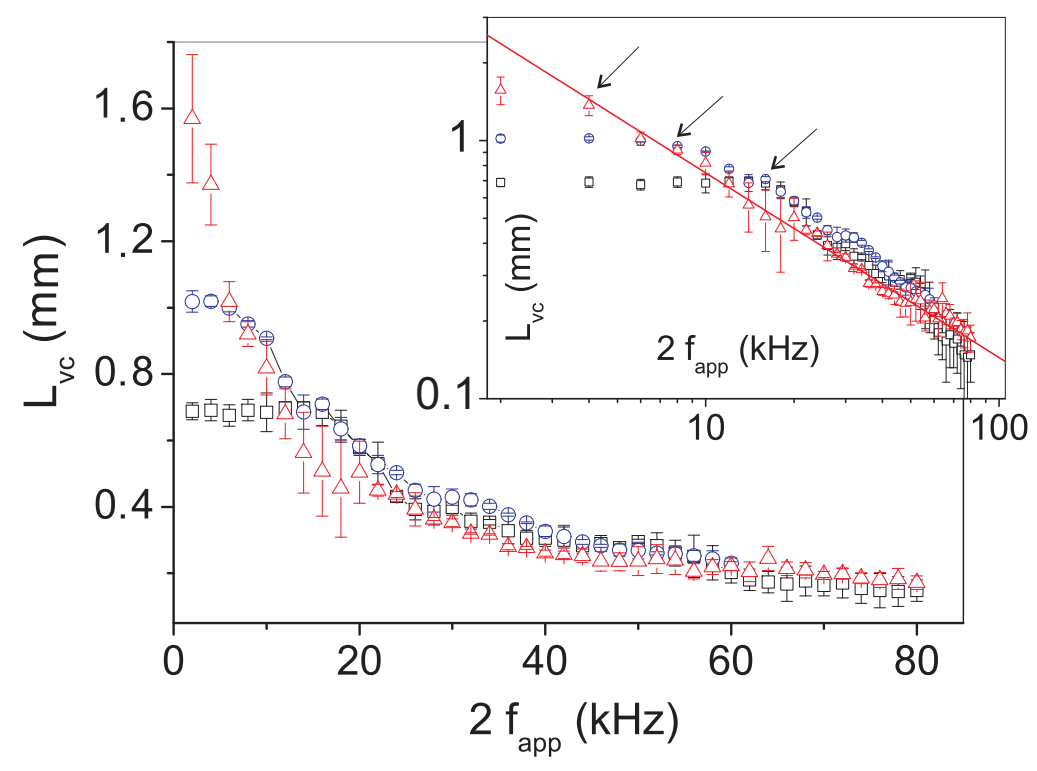

Figure 5.12: The displacement of the vortex center $L_{v c}$ versus frequency for droplets of different volume $(\triangle: 1 \mu \mathrm{L}, \bigcirc: 0.7 \mu \mathrm{L}$ and $\square: 0.4 \mu \mathrm{L})$. The inset shows the same plot in log-log scale. The red line is the fit with slope -0.7 which is close to the anticipated value $-2 / 3$. The actual driving frequency is two times the applied frequency (see chapter 1).

The periodic electrostatic forces at the contact line generate capillary oscillations of the liquid-air interface. Consequently, a region of liquid within the penetration length of these capillary waves is put into periodic motion. As we discussed in part I, the periodically oscillating liquid also experiences an aperiodic drift due to the broken symmetry of the stress oscillations caused by the pinning site. This drift appears as the flow vortices over many oscillation cycles. We assume that the decreasing penetration length with increasing frequency causes the regions of liquid oscillation (periodic plus the drift) inside the drop shift towards the contact line. Therefore the vortices shift towards the contact line near the pinning site where the nonlinear effects causing the drift exist.

If shifting of the vortices occurs due to the decreasing penetration 
depth of the capillary waves, then the vortex position $L_{v c}$ and the penetration depth must at least show the same scaling behaviour because both originate from stress variations at the liquid-air interface. In the inset of Fig. 5.12, $L_{v c}$ is fitted between the onset frequency (indicated by the arrow) and a frequency above which vortices do not shift ( $\left.2 f_{\text {app }}=50 \mathrm{kHz}\right)$. It gives the exponent as $-0.70 \pm 0.1$. This scaling, $L_{v c} \sim f_{a p p}^{-2 / 3}$ therefore can be compared with the decrease of the penetration depth $\left(\sim q^{-1}\right.$, where $q$ is the wave number) of capillary waves with increasing angular frequency $\omega$, i.e.: $q^{3} \sim \omega^{2}[116]$.

Our results do not strictly depend on the exact values of the frequency at the starting and the ending of the frequency sweep. The starting frequency must be above about $100 \mathrm{~Hz}$ where the hydrodynamic flows begin to exist in an electrowetting drop and below the onset frequency for shifting of the vortices (about $4 \mathrm{kHz}$ for a drop with $1 \mathrm{~mm}$ radius, as seen from Fig. 5.12). The end sweeping frequency must be few tens of kilo Hertz to achieve maximum intensity enhancement. Choosing a very high end frequency with extremely low salt concentration will generate electro thermal flows inside the drop [63, 62]. With $10 \mathrm{mM}$ salt concentration (conductivity $\sim 0.14 \mathrm{~S} / \mathrm{m}$ ) an electro thermal flow of order $\mathrm{mm} / \mathrm{s}$ requires about $1000 \mathrm{kHz}$. Therefore in our measurements, any electrothermal effects can be ruled out. Moreover we obtained similar shifting of flow vortices and pre-concentration even at $200 \mathrm{mM} \mathrm{KCl}$ concentration. These results point out that the particle accumulation is not driven by any electric fields inside the drop but by a pure hydrodynamic process. We assume that the particles are preferentially concentrated [117] at the center of the vortices by inertial effects. Near the center of the vortices slowly moving particles cluster together and consequently feel increased inertial effects. This is consistent with our observation that $20 \mathrm{~nm}$ particles always form very small amount accumulation (Fig. 5.9). This is because the inertial effects on these light weight particles are very small.

By applying a voltage at a fixed high frequency (say, $50 \mathrm{kHz}$ without any sweeping) produces small vortices near the pinning site with few number of particles in them. The resulting intensity enhancement is com- 
parable to that of a very fast sweep rate in Fig. 5.11. This technique requires a frequency sweep and a resulting slow shifting of the vortices towards the contact line to obtain maximum concentration enhancement. During this slow shifting, more particles are collected in the vortex centres.

The partial evaporation of the drop during the pre-concentration does not affect the results. The experiments in saturated atmosphere around the drop to prevent evaporation also gave similar results.

Our pre-concentration technique is suitable when analyte (for example particles, cells or DNA) in the drop need to be collected together for better detection and analysis. The technique can be integrated in droplet based microfluidics preferably in applications using single or an array of sessile drops. The system can be easily integrated in a chip or as an array of drops thanks to the two properties: (i) the long metallic stripe patterned on the substrate can be used as the wire for applying voltage and also as the pinning sites for an array of drops, (ii) the positioning of drops on the metallic stripe is not very critical because the pinning can be anywhere on the contact line of the drop. This facilitates easy deposition of drops onto the substrate, for example by ink jet printing methods. Moreover, the concentration enhancement near the electrode may help electro-chemical detection/analyses of the sample.

There are certain issues to be solved in order to ameliorate the performance of the technique. It is affected by the bio-molecular adsorption which is a bottleneck in electrowetting based biofluidic chips. For example, in our measurements with DNA, the adsorption caused DNA to be collected at random places on the SU8 surface. Experiments with green fluorescent protein (GFP) were hindered with strong adsorption effects. Adsorption causes the contact line strongly pinned on the surface, preventing its oscillations. Such non-specific adsorption is manifested by hydrophobic interactions. Tuning the substrate properties, or $\mathrm{pH}$ of the working liquid may help in reducing the biomolecular adsorption effects [118]. 


\subsection{Conclusion}

In conclusion, we have demonstrated a technique to pre-concentrate sample in a sessile drop. By sweeping the frequency of the applied voltage in an electrowetting sessile drop with locally pinned contact line, the internal flow vortices can be moved towards the pinning site at the contact line. The moving flow vortices accumulate solute particles near the contact line. This technique can be used to concentrate particles or bio-molecules locally inside a sessile droplet. 



\section{Chapter 6}

\section{Suppressing the coffee stain effect by electrowetting}

When a drop containing nonvolatile solutes evaporates on a solid surface, an unevenly distributed residue pattern is observed. This phenomenon is called coffee stain effect. Electrowetting found to suppress stains of both colloidal particles of various sizes and DNA solutions at alternating frequencies ranging from a few Hertz to a few tens of $\mathrm{kHz}$. The method is not explored in depth. Here we present an investigation to identify the potentials and limitations of the method. Influence of factors such as amplitude of the contact line motion, solute particle concentration and their size, are evaluated on the success of coffee stain effect suppression. Finally, in order to emphasize the importance of suppressing coffee stain effect, we present a MALDI-TOF experiment of suppressed and heterogeneous residues of poly ethylene glycol. A sharp spot of residue gave two order increase in the signal strength. 


\subsection{Introduction}

Evaporation of a sessile drop containing non-volatile solutes leaves behind a ring like, spatially heterogeneous residue called coffee stain. Deegan et al. pointed out that the physical origin of the coffee stain effect is a combination of pinning of the three-phase contact line and the convective flux driven by evaporation [119].

The formation of coffee stains is often encountered as an undesirable aspect in industrial applications. For instance, coffee stains compromise the overall performance of ink jet printing of complex fluids, painting, DNA microarrays $[120,121]$ or other bio-chemical analyses like MALDITOF [122] that involve drying of sessile drops. Suppressing the coffee stains is very important in such applications.

Various methods have been proposed in the literature to control self assembly and pattern formation in evaporating drops especially to suppress the coffee stain effect. Introducing surface tension gradients driven 'Marangoni flows' [123], modifying the relative thermal conductivities of the substrate and liquid [124], complex electrode geometries to induce electro osmotic flow [125] and using hydrophobic substrates [120] have been proposed to counteract the accumulation of particles at the contact line. The above mentioned methods require either chemical additives or electric fields in the drop. Alternatively a dynamic and non-invasive method based on electrowetting [19] can be used to suppress coffee stain effect [64]. Two main effects contribute to the suppression: (i) the strong internal azimuthal flows (see also chapter 5) in a sessile drop generated by AC electrowetting counteract the evaporation driven flux, thereby prevent the accumulation of solute particles at the contact line and (ii) the periodic electrostatic force at the contact line which prevents pinning [131]. In the course of evaporation, the competition between the pinning due to the solute particle deposition at the contact line and it's de-pinning due to the contact line oscillations determines the success of the suppression.

In this chapter, we show that electrowetting can suppress coffee stains of both colloidal particles of various sizes and DNA solutions at AC fre- 
quencies ranging from few $\mathrm{Hz}$ to few tens of $\mathrm{kHz}$. Moreover, we study different factors which influence the suppressing. We identified that the initial solute particle concentration, their size and the applied voltage are the three major factors determining the suppression of coffee stains. Further, we found that there exists a maximum initial particle concentration above which the coffee stains can not be suppressed by electrowetting. This maximum initial concentration is a function of the particle size and the applied voltage.

\subsection{Experimental methods}

Our experimental setup (Fig. 6.1) consists of a sessile drop placed on an SU8 layer (of $5 \mu \mathrm{m}$ thick) spin coated on an ITO (Indium-Tin-Oxide) covered glass. The SU8 layer has an advancing and receding contact angle of $85^{\circ} \pm 3^{\circ}$ and $60^{\circ} \pm 3^{\circ}$ respectively. An AC voltage was applied between the droplet and the ITO layer by inserting a platinum wire of 50 $\mu \mathrm{m}$ in diameter in the drop.

To demonstrate the suppression effect, we used sessile drops containing colloidal particles or cDNA. The colloidal suspensions of initial particle concentration $0.05 \%$ (by volume) were prepared by adding carboxyl terminated fluorescent labelled polystyrene particles (Polysciences.Inc) to Millipore water with $10 \mathrm{mM} \mathrm{LiCl}$ salt. The diameter of the particles ranged from $5 \mu \mathrm{m}$ to $0.1 \mu \mathrm{m}$. The DNA solution of $2.5 \mathrm{ng} / \mu \mathrm{L}$ concentration was prepared with Millipore water. For imaging purposes, the diluted cDNA was labelled with green fluorescent nucleic acid stain (SYTO 24) to a ratio $4: 1$. The applied voltage was $200 \mathrm{~V}$ (root mean square value) at frequency ranging $6 \mathrm{~Hz}$ to $100 \mathrm{kHz}$. In order to study the suppression

effect as a function of the initial particle concentration and their size, we used particles of diameter ranging from $3 \mu \mathrm{m}$ to $20 \mathrm{~nm}$ with an initial particle concentration $\phi_{c}$ ranging from 0.005 to $0.6 \%$ by volume. The drops had an initial volume of volume $0.5 \mu \mathrm{L}$. The root mean square amplitude of the applied voltage ranged from $0-350 \mathrm{~V}$ at a frequency of $2 \mathrm{kHz}$. In addition, an amplitude modulation with a frequency of $100 \mathrm{~Hz}$ was ap- 


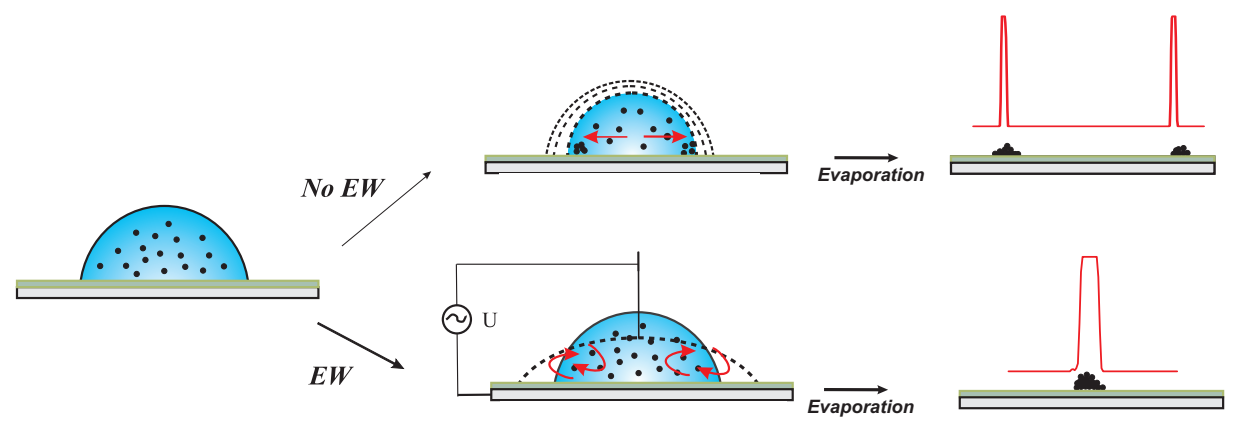

Figure 6.1: Schematic of a drop evaporation process. Top row represents evaporation without electrowetting and with contact line pinning. Bottom panels show the process with electrowetting and with no contact line pinning. Right column shows schematic and experimental intensity cross sections (solid lines) through the residue.

plied. While keeping the voltage on, the drop was allowed to evaporate completely and the residue was recorded using a confocal microscope.

All the experiments were also conducted at zero applied voltage for reference. To measure the flow speed inside the drop and to record the contact line oscillations, we used a high speed camera (Photron SA3) and an inverted microscope. The flow speed was determined by tracking the particles inside the drop as described in chapter 2. Evolution of the contact angle during evaporation was measured using drops of volume $1 \mu \mathrm{L}$ containing polystyrene particles of diameter $200 \mathrm{~nm}$ at different initial concentrations ranging from 0.0007 to $0.4 \%$ by volume. All the experiments were done at ambient temperature, $23 \pm 2^{\circ}$.

\subsection{Results and discussion}

We start with demonstrating the suppression of the coffee stain effect with different solute materials in the sessile drops. In Fig. 6.2, the images of the residues after the drop evaporation are shown. When the drops evaporate undisturbed, they form ring like coffee stains. The evaporation of the 


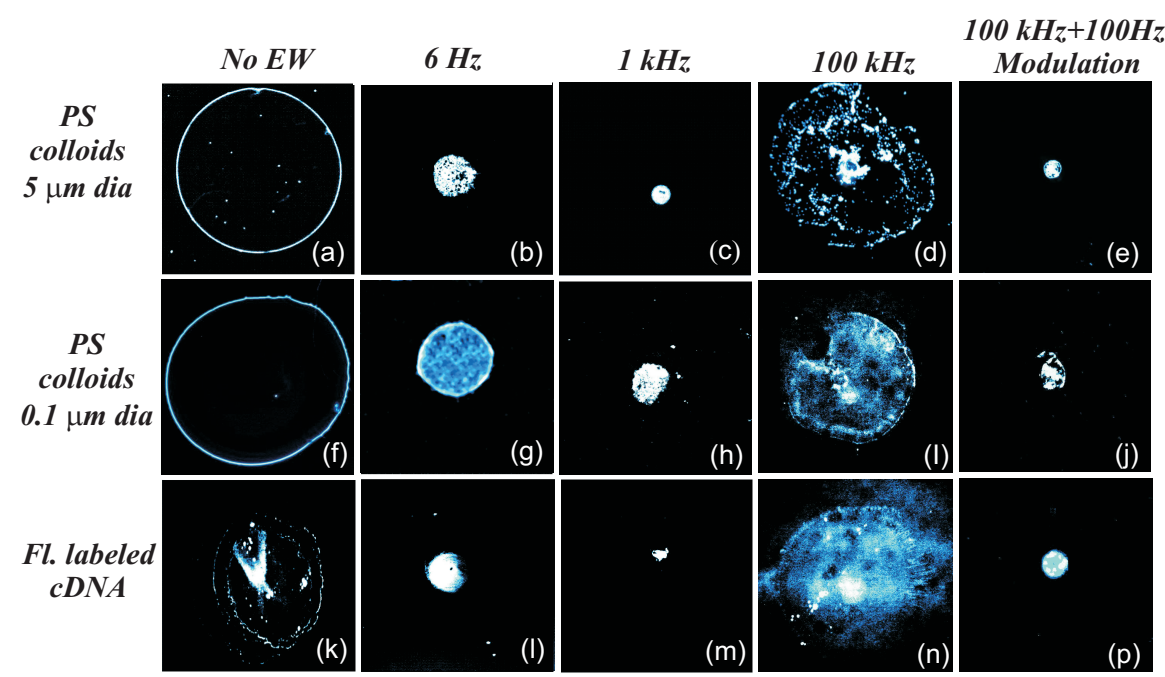

Figure 6.2: The residual patterns of various materials after evaporation under undisturbed case and under electrowetting at three different frequency regimes $(6 \mathrm{~Hz}, 1 \mathrm{kHz}$, $100 \mathrm{kHz}$ and $100 \mathrm{kHz}$ plus $100 \mathrm{~Hz}$ amplitude modulation). The solute materials and the frequency ranges are shown in the figure.

solvent from the drop causes the surface (including the contact line) simply recede according to the mass lost as vapour phase. However, when the contact line is pinned at surface defects, mass conservation requires that additional solvent is transported from the center of the drop towards the contact line. This flux carries particles along and deposits at the contact line increasing the pinning forces. It ultimately forms a ring shaped solid residue.

By using electrowetting, pinned contact line can be set into motion by applying an electric force that exceeds the maximum pinning force which is related to the macroscopic contact angle hysteresis. A time varying force at the contact line constantly depins it from the defects. This constant depinning during evaporation leads to a spot like residue (second column of Fig. 6.2). 
The solute particles, accumulating at the contact line during evaporation enhance the pinning forces. Therefore it is beneficial to decrease the deposition of the particles at the contact line by counteracting the evaporation driven flow fields in the drop. Electrowetting generates flow fields due to the oscillations of the drop (see chapter 5). For instance, at a frequency of $1 \mathrm{kHz}$ of the applied voltage, azimuthal flow fields, generated inside the drop minimize the particle deposition at the contact line. Additional oscillations of the contact line depin it from the surface defects. This combined effect even decreases the size of the residue formed after evaporation (see the third column of Fig. 6.2).

Choosing a particular value for the frequency (for example $1 \mathrm{kHz}$ for the above mentioned experiments) is not critical. The frequency must be small to make large amplitude oscillations to the contact line. Because, with increasing frequency the amplitude of these oscillations decreases (see Fig. 6.3A). The frequency must be also high enough to maintain strong and stable internal flows in the drop to prevent particle deposition at the contact line. By choosing a frequency between $100 \mathrm{~Hz}$ and 5 $\mathrm{kHz}$, flows of magnitude $\mathrm{mm} / \mathrm{s}$ can be ensured in the drop. Such flows are strong enough to counteract the evaporation driven radial convection which is typically of $50 \mu \mathrm{m} / \mathrm{s}$ in magnitude [64]. At extremely high frequencies, the drop cannot follow the driving force at the contact line and the internal flows dwindle away (see Fig. 6.3B). This results in an unsuppressed state of the coffee stains as seen in Fig. 6.2 (at $100 \mathrm{kHz}$ ). By applying an additional $100 \mathrm{~Hz}$ amplitude modulation to the voltage of frequency $100 \mathrm{kHz}$, the contact line oscillations again suppress the coffee stains (see the fifth column in Fig. 6.2). As seen in Fig. 6.3A, an amplitude modulation at $100 \mathrm{~Hz}$ causes the contact line to oscillate with an amplitude $\left(A_{T C L}\right)$ of $125 \pm 40 \mu \mathrm{m}$ which is good enough to depin from macroscopic and microscopic pinning sites. The periodic fluctuations in contact line and in flow speed in Fig. 6.3 are due to the increased amplitude of oscillations at the resonant frequencies of the drop. A detailed explanation over the correlation between the resonant behaviour of oscillating drops and internal flow speeds is provided in chapter 5 . 

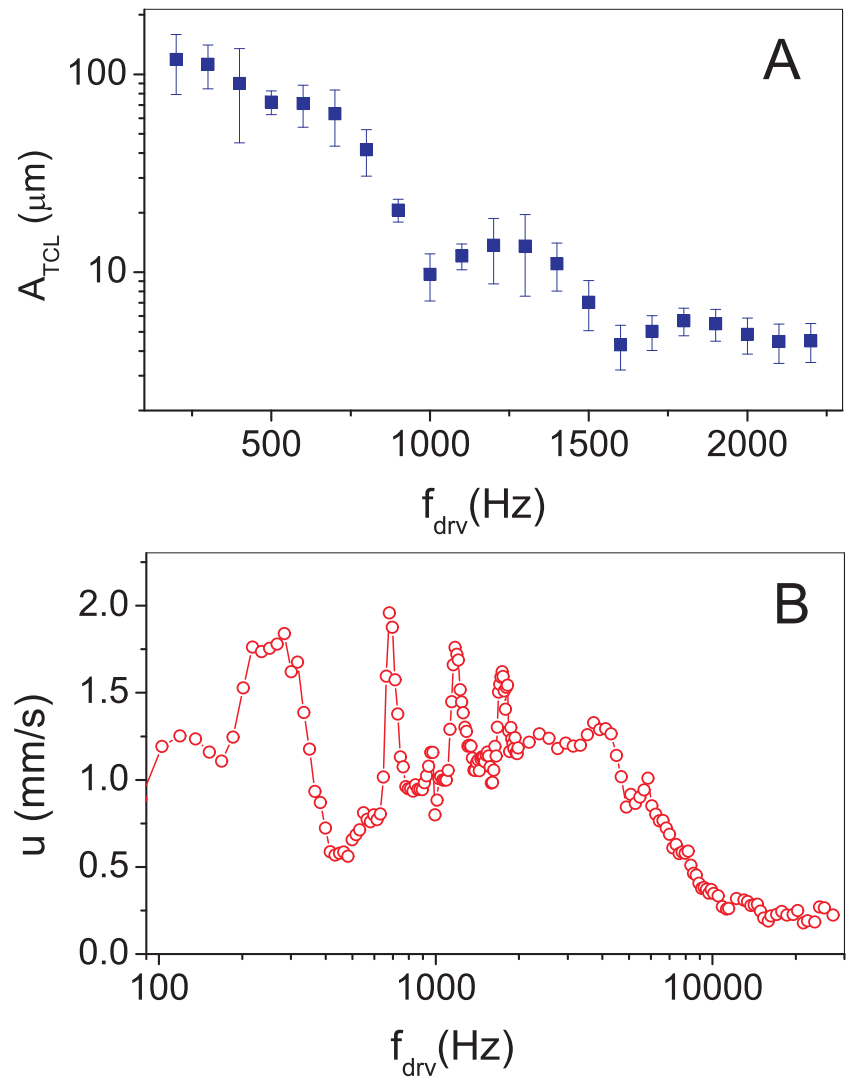

Figure 6.3: The amplitude of oscillation of the contact line, $A_{T C L}(\mathrm{~A})$ and the magnitude of flow speed, $u(\mathrm{~B})$ versus the driving frequency $f_{d r v}=2 f_{a p p}$ where $f_{a p p}$ is the actual applied frequency (see also chapter 1). The rms voltage was kept at $190 \mathrm{~V}$. The fluctuations in the curves are due to the resonant oscillations of the drop.

To prevail over the pinning situation, not just the frequency but the strength of the applied voltage can be tuned as well. Above a particular voltage, the electric force at the contact line can overcome the pinning force. Above this voltage the contact line starts to move. The magnitude of $A_{T C L}$ and flow speed $u$ increases with increasing voltage. However they seem to saturate at large applied voltages (Fig. 6.4). We attribute 


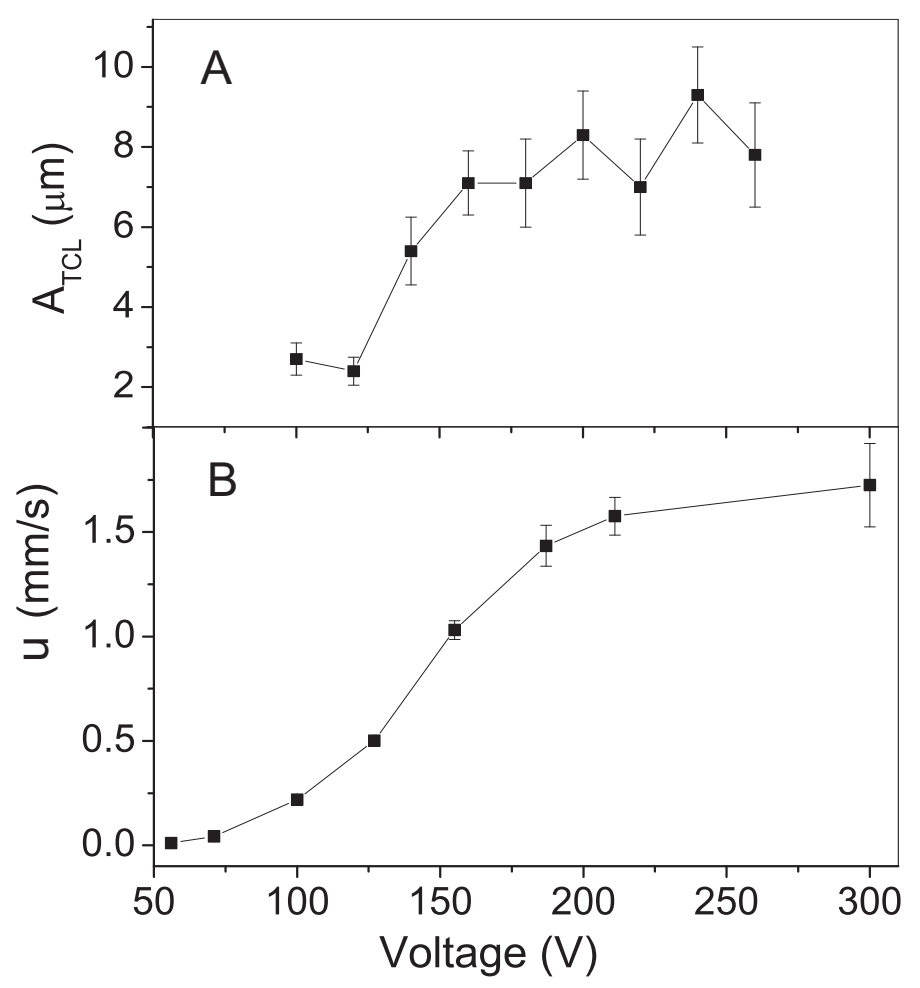

Figure 6.4: The amplitude of oscillation $A_{T C L}$ of the contact line (A) and the magnitude of flow speed $u(\mathrm{~B})$ versus the applied voltage at frequency $1 \mathrm{kHz}$. Both $A_{T C L}$ and $u$ saturate at large voltage.

this to the contact angle saturation [19] effects in electrowetting occurring above a voltage $V_{\text {sat }}$. In our measurements the $V_{\text {sat }}$ was about $275 \pm 25 \mathrm{~V}$.

The above mentioned observations help to determine the optimum strength and frequency of the applied voltage. Even in the ideal driving conditions, the constituents of the drop may influence the suppression of the coffee stains. For instance, we demonstrate the coffee stain phenomenon and it's suppression at different initial particle concentration, $\phi_{c}$. In Fig. 6.5 the images of the residues formed by evaporating drops at zero voltage are shown in the top panels. Similarly, the residues formed 
upon evaporation under electrowetting are shown in the bottom panels. Depending up on the magnitude of $\phi_{c}$, a spot or a big ring is formed after evaporation under electrowetting. Due to the moderate hydrophobicity of SU8, the rings are smaller than the periphery of the drop before evaporation. Application of the voltage results in the spreading of the drop which leads to bigger rings after an unsuccessful suppression (the third column). At relatively small $\phi_{c}$, a successful suppression produced a sharp spot of residue (the first column).

To understand the correlation between pinning of the contact line and $\phi_{c}$, we start with evaporation of the drops without electrowetting. Evolution of the contact angle and the base diameter goes through different phases during evaporation. The process has in general three phases [126, 127]: (i) decreasing contact angle (fixed contact line), (ii) constant contact angle (receding contact line) and (iii) receding contact line with sharp decrease in contact angle (Fig. 6.6).

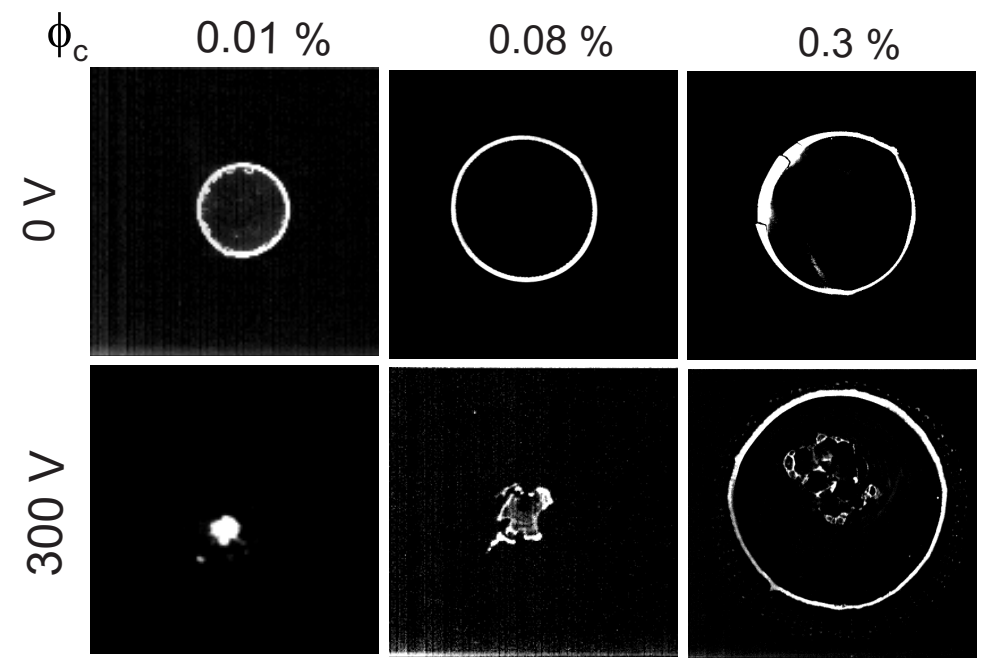

Figure 6.5: The images of the residues after the evaporation with (bottom panels) and without (top panels) electrowetting at three different initial particles concentrations $\phi_{c}$ (particle size: $100 \mathrm{~nm}$ ). 


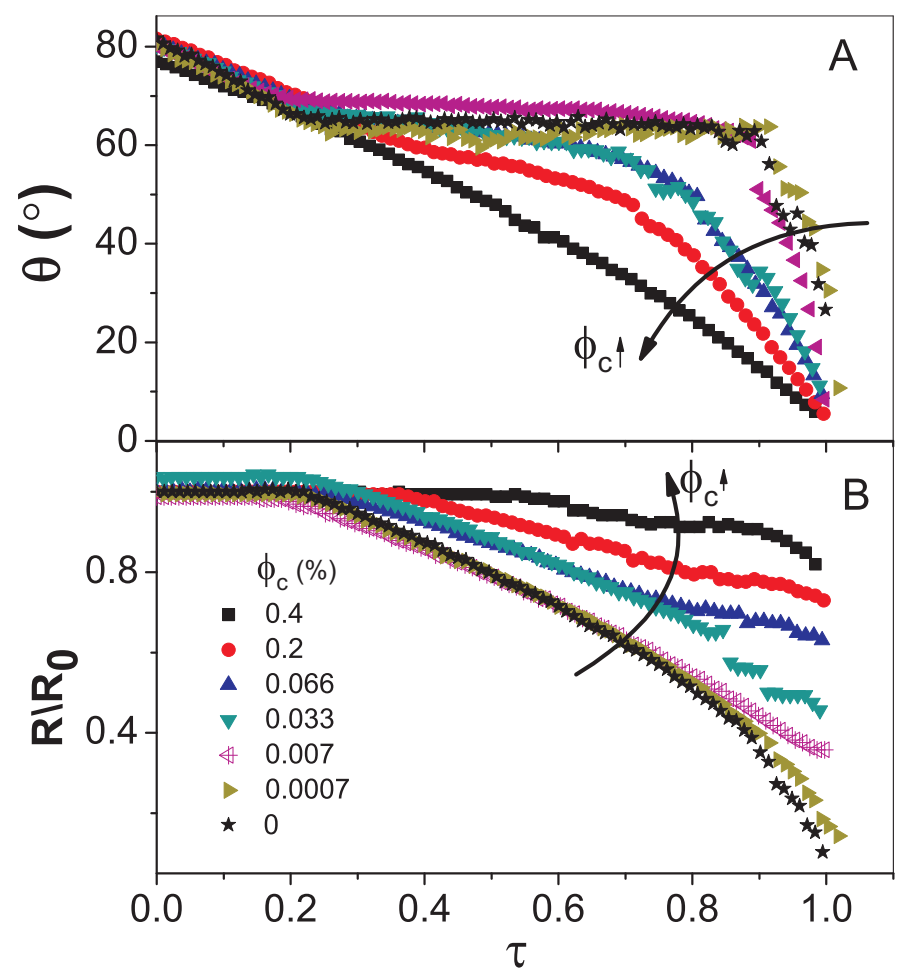

Figure 6.6: The contact angle (A) and the base radius (B) versus time for different initial particle concentration $\phi_{c}$. The $R$ is normalized by the initial base radius of the drop $R_{0}$ and time is normalized with respect to the time required for the complete evaporation of the drop. $\phi_{c}$ is the (volume) concentration of the particles in the drop (particle size: $200 \mathrm{~nm})$.

Regardless of the exact phase of the drop during evaporation, the particles are being deposited at the contact line. The amount of particles deposited at the contact line increases with the initial amount of particles in the drop. The time scales of the different phases are determined by the pinning of the contact line. The substrate itself cannot keep the contact line pinned. The pinning of the contact line is self induced i.e. by the deposition of particles in the evaporating drop [128]. By increasing $\phi_{c}$ we observe early pinning of the contact line characterized by vanishing 


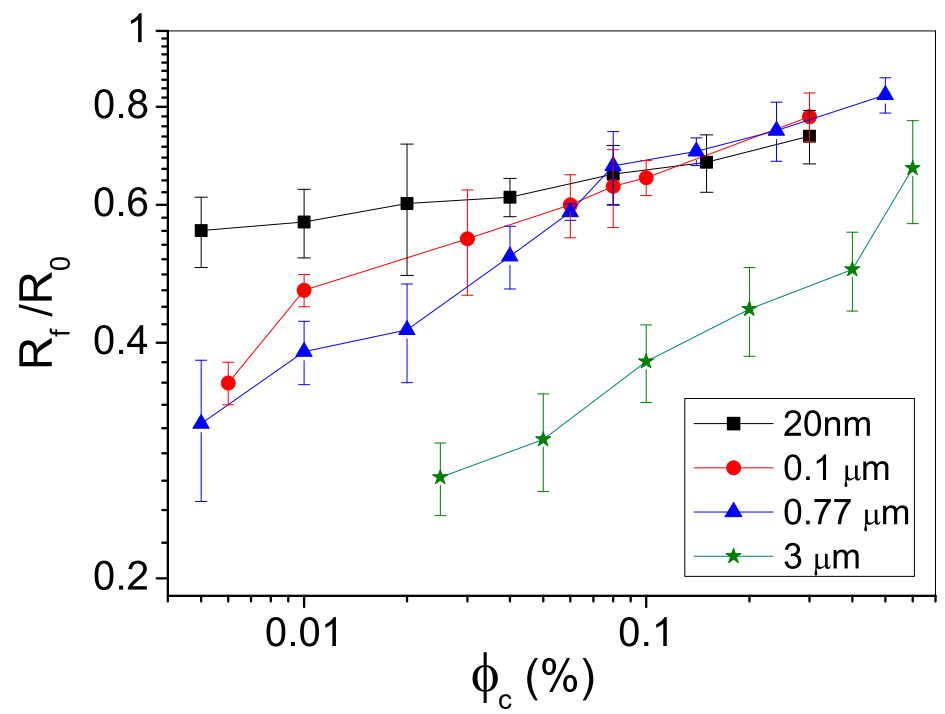

Figure 6.7: The radius $R_{f}$ of the coffee rings versus particle concentration $\phi_{c} . R_{f}$ is normalized by the initial base radius $R_{0}$ of the drop. The size of the rings increases with increasing $\phi_{c}$. The small particles form bigger rings.

the second and the third phases. This is because by increasing $\phi_{c}$ the volume of deposition of the particles at the contact line increases [129, 130]. Early pinning of the contact line results in the formation of bigger rings. This is clear from Fig. 6.7, where the radius of the ring $R_{f}$, normalized by the initial radius of the drop $R_{0}$, is plotted as a function of $\phi_{c}$. Moreover the drops with smaller particles form bigger rings. For example, the rings formed from $20 \mathrm{~nm}$ particles are bigger than those from $3 \mu \mathrm{m}$ particles at the same $\phi_{c}$. We assume that the small particles are easily transported into the wedge shaped contact line region (in micro scale) by convection. At the evaporating wedge region, the attractive capillary forces due to the menisci formed around the particles cause the formation of crystalline structures [132]. A recent study [133] reports that the dominant mecha- 


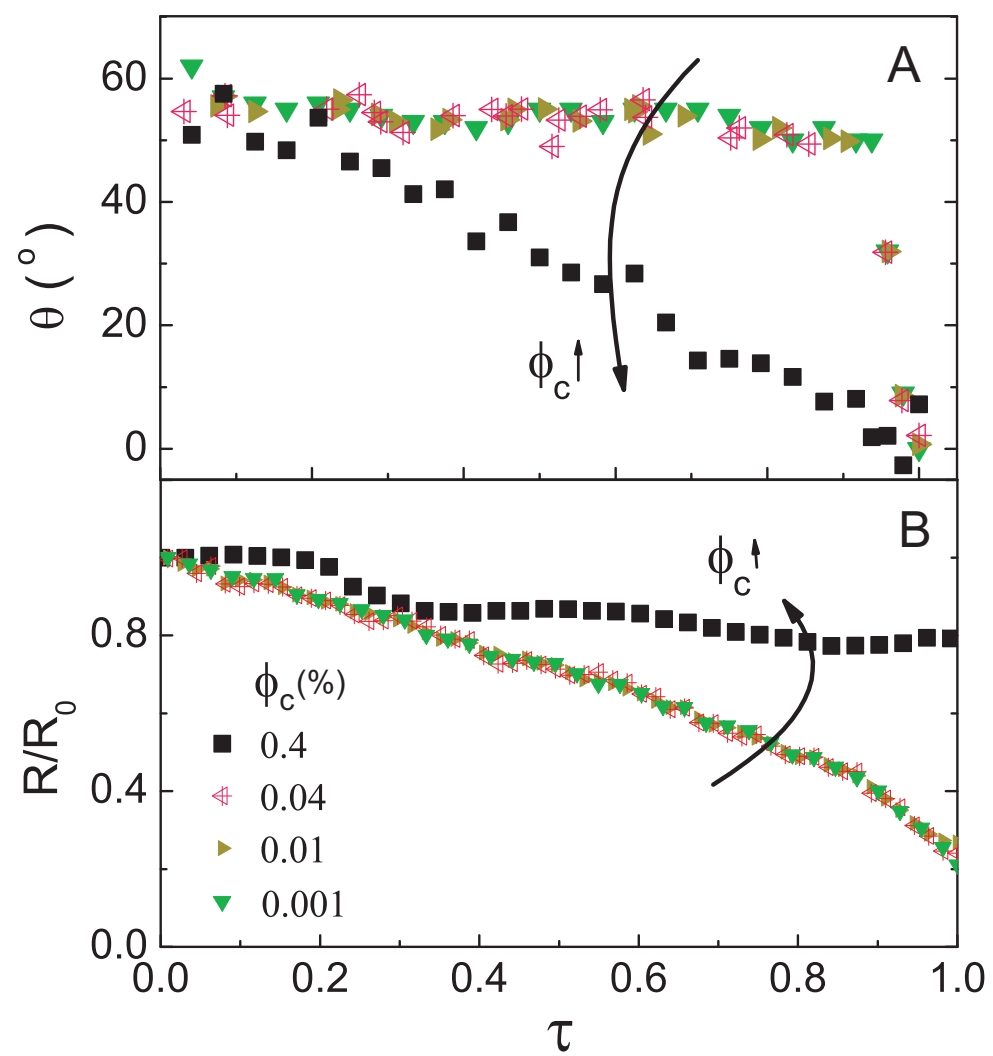

Figure 6.8: The contact angle (A) and base radius (B) versus time for a drop under AC electrowetting. The $R$ is normalized by the initial radius of the drop $R_{0}$ and time is normalized with respect to the time required for the complete evaporation of the drop. $\phi_{c}$ is the (volume) concentration of the particles in the drop (particle size: $200 \mathrm{~nm}$ ).

nism for the self structuring of the particles in the confines of the wedge surfaces is the stagnation flow and not the capillary attraction between the particles. Whatever be the dominant mechanism, the ordering of the particles at the wedge region produces strong pinning effects.

In the scenario where $\mathrm{AC}$ electrowetting is applied, the drop behaves in a different way. The contact line is in a periodic motion preventing any pinning. Therefore, the macroscopic contact angle stays constant (while 


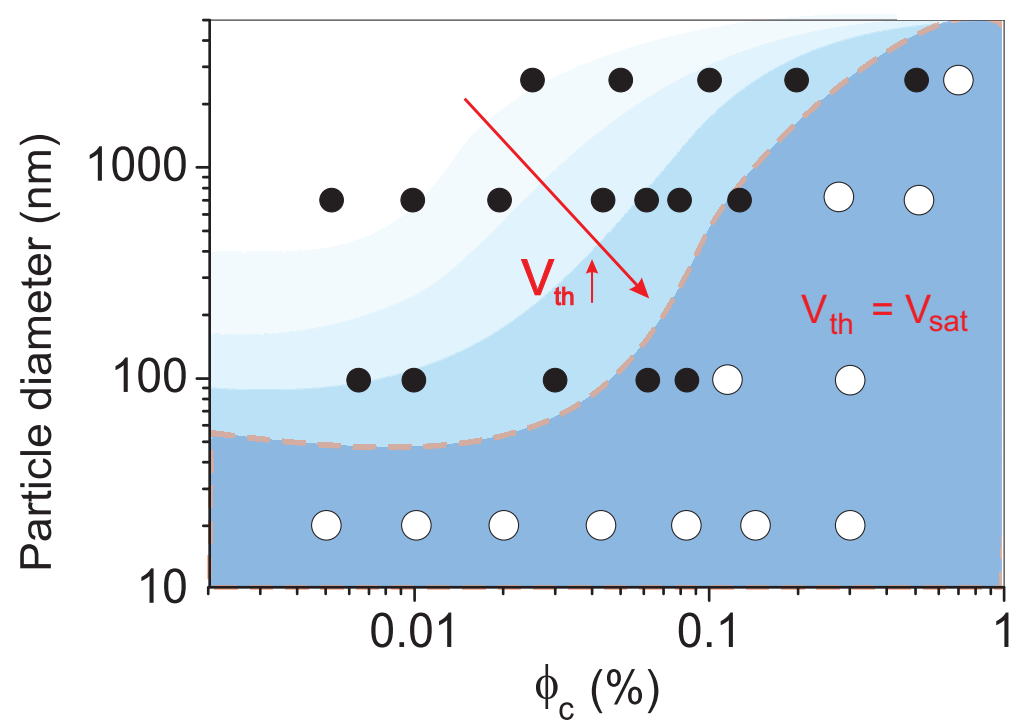

Figure 6.9: A phase diagram giving the success of the suppression of stains. Open circles and filled circles represent unsuppressed (rings) and suppressed (spots) states respectively. The blue shades and the red dash line (both are only guide to eyes) represent increasing threshold voltage $V_{t h}$ and the saturation voltage of electrowetting.

the base radius decreases) during evaporation as shown in Fig. 6.8. The evaporation of the solvent increases the particle concentration in the remaining drop. This causes pinning in a later stage of the evaporation. Increasing $\phi_{c}$ results in an early pinning of the contact line and forms bigger rings. The unsuccessful suppression at large $\phi_{c}$ implies that the depinning effects from the contact line oscillations are not sufficient to overcome the strong pinning due to the particle accumulation. If the oscillations are not strong enough to depin the contact line, the electrowetting itself helps particles to form crystalline structures. The spreading of the contact line region due to electrowetting enhances evaporation driven particle flux and crystal assembly rate [134].

Hitherto we considered different scenarios of pinning and means of achieving strong depinning of the contact line. We now go back to the 
experiments where drops with particles at different initial concentration and their size are evaporated under electrowetting. The results are presented in Fig. 6.9. The filled circles represent suppressed states i.e., sharp spots of residue. The open circles represent unsuppressed states, i.e., extended stains. Upon increasing $\phi_{c}$ or decreasing particle size, the contact line is more prone to pinning on the substrate (also see Fig. 6.7), requiring higher threshold voltage to depin. The increasing threshold voltage is represented as blue coloured shades (guide to eyes) in Fig. 6.9. Enhanced pinning effects with increasing $\phi_{c}$, cannot be overcome by increasing the voltage because the maximum applied voltage is limited by the instabilities at the contact line [19] and the dielectric breakdown of the SU8 layer. In addition, the contact angle saturation can be also a limiting factor (see Fig. 6.4).

Overall the suppression of coffee stain effect occurs at wide range of particle sizes and corresponding initial particle concentrations. In biological or chemical detection methods suppression of coffee stain effect is required when the initial concentration of the analyte is very small. For instance in MALDI-TOF analyses [122], when a chemical compound which is present in very small concentration, suppression of the stains becomes a vital requirement. This is because ionization (using a laser beam) of a heterogeneous residue produces many weak and temporally spread signals while that of a single sharp spot of residue gives a strong signal at once. This aspect is clear from the ion count versus time plot in a MALDI-TOF experiment of poly ethylene glycol (PEG) shown in Fig. 6.10. Time axis is the scanning time of the laser beam. When PEG is distributed heterogeneously the signal is temporally spread and its strength is low (Fig. 6.10A). On the other hand, when a spot of PEG (obtained by suppressing coffee stain effect by electrowetting) is ionized, the signal strength is increased by an order of two (Fig. 6.10B). This is because, the spot of the PEG was ionized at once.

The success rate of the technique can be extended by using substrates with low contact angle hysteresis. As observed from Fig. 6.3, the broad frequency range of voltage, that can be applied to produce strong mixing 


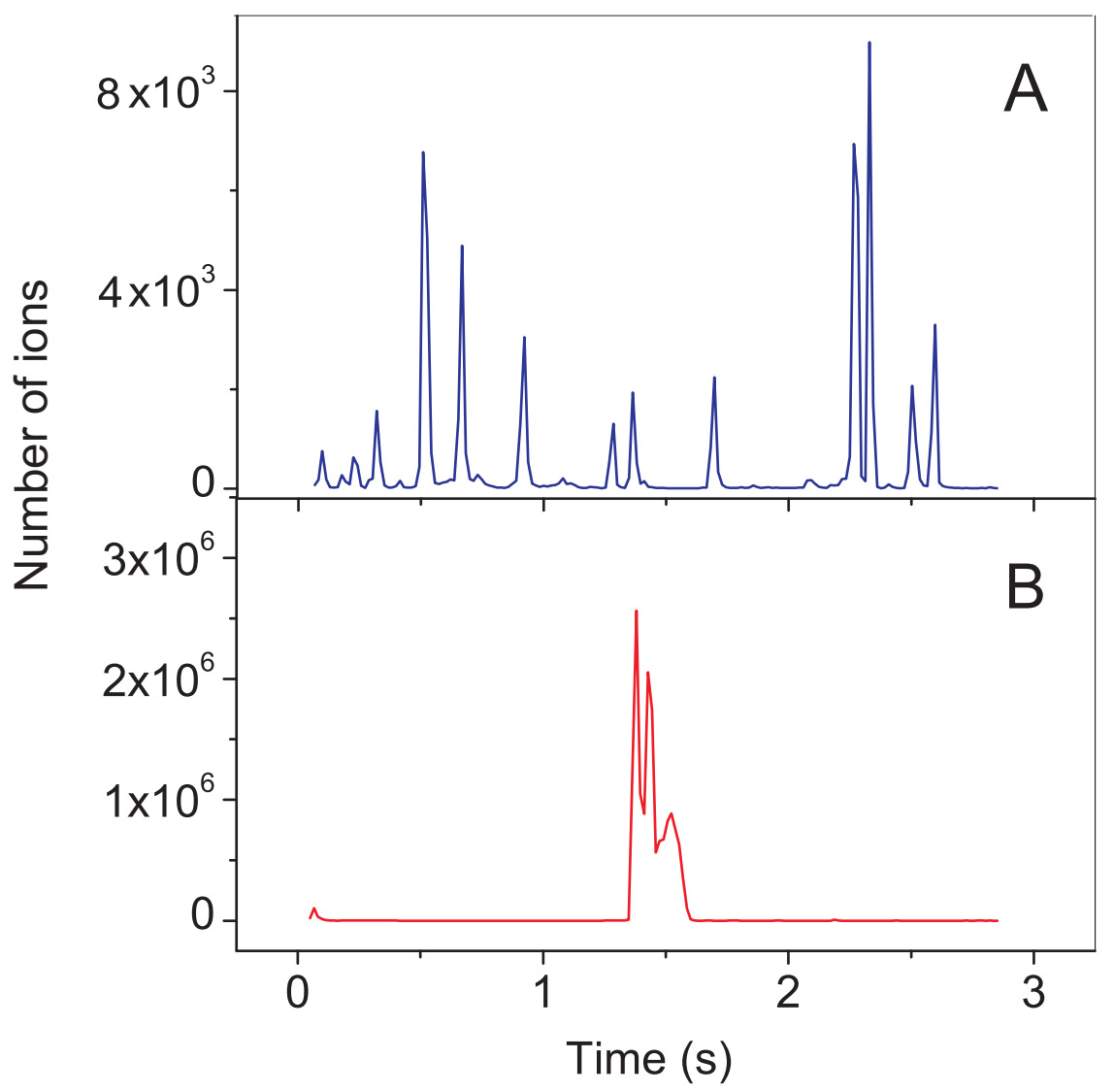

Figure 6.10: Number of ions detected versus scanning time in a MALDI-TOF experiment with poly ethylene glycol (PEG). A) The peaks are spread in time due to the inhomogeneous distribution of PEG caused by the coffee stain effect. B) When the coffee stain effect is suppressed the ion count (red solid line) is increased by an order of two.

flows and contact line oscillations shows the flexibility of the method. This gives possibility of further investigations by applying arbitrary or combination of waveforms to shake the contact line in a random manner to explore better depinning effects. 


\subsection{Conclusion}

In conclusion, we have considered different aspects of suppression of coffee stain effect by electrowetting. An applied voltage with a wide range of frequencies can be applied to drive the drop. The success of suppression of coffee stain effect by electrowetting depends on the particle size, their initial concentration inside the drop and the strength of the applied voltage. The suppression occurs for a broad range of particle sizes. The method is most efficient at low initial concentrations of analyte (e.g. particles) where sharp spots of residues are required for better detection and analysis. 


\section{Chapter 7}

\section{Oscillations of a sandwiched drop by electrowetting}

We sandwich a drop between two hydrophobic glass plates and periodically modulate the contact angle by AC electrowetting. The drop oscillates in different shape modes depending up on the frequency of the applied voltage. The oscillations have half the excitation frequency of the contact line, i.e. with the frequency of the applied voltage. The drop response for a sinusoidal driving, is 'stop and go' with substantial deviation from sinusoidal behaviour especially at lower oscillation modes. Although the shape modes follow our simple theoretical model, the resonances appear at substantially lower frequencies than expected. We attribute this to the pinning effects of the contact line on the substrate. 


\subsection{Introduction}

The first quantitative study of oscillations of free liquid drops was presented in the pioneering work of Rayleigh [109]. The study of oscillations of free drops $[135,136]$ has fundamental and technological significances. For example, liquid drops are paradigm models in nuclear physics [137] or in astrophysics [138]. Knowledge of drop dynamics can help technological fields where micro droplets are generated, such as in ink-jet printing or fuel injection. Emergence of microfluidics brought great scientific attention to the oscillations of sessile drops [139]. In contrast to oscillating free droplets the oscillating sessile droplets behave in a different way. Their dynamics are affected by the contact angle [136] or pinning of the three phase contact line on the supporting surface [111]. In microfluidics, sessile drop oscillations are important in mixing [58] especially by controlling the flow patterns in them (chapter 5) or controlling the drying patterns of evaporating sessile droplets [64].

In discrete droplet based microfluidics, one often encounters flattened droplets for example, sandwiched between two rigid plates. The dynamics of sandwiched and gravitationally flattened drop (puddle) are similar. The spontaneous oscillations of puddles on a hot surface were first reported in 1952 by Norman J. Holter [140]. Later various other authors $[141,142,143,144,116,145,146,147]$ studied it in detail. Puddle oscillations are often produced by a periodic acceleration field applied to the droplet. The periodic acceleration is equivalent to that of a varying gravity. On the other hand, the gravity determines the height $(H)$ of the puddle, i.e. $H=2 l_{c}$, where $l_{c}$ is the capillary length. When the contact line is pinned only weakly to the substrate, it's radius $R$ becomes periodic in order to conserve the volume. The time varying radius causes the resonance frequency for the free oscillations modulated in time which leads to parametric forcing [144]. The drop responds to this parametric forcing at half the frequency of excitation. These oscillations are a consequence of $R \gg H$ and is observed only in flat drops. The oscillations of flat drops are often called triplon modes or star drop oscillations. 
In this chapter we show that a sandwiched drop between two hydrophobic plates can be excited by AC electrowetting. The small asymmetry in the drop periphery, for instance by a microscopic pinning site, determines the initial direction of oscillations. We excite different azimuthal modes by driving the drop at different frequencies of the applied voltage. Our experimental results match with our simple theoretical model that excludes any nonlinear effects. However the eigen modes of oscillations appeared at substantially lower frequencies as compared to the ones predicted by the theory. This deviation in the resonance frequencies was different for sample to sample however small when low hysteresis substrates were used. Moreover the deviations systematically decreased with increasing eigen modes. The oscillations are very sensitive to the pinning on the substrate and the drop showed 'stop and go' type oscillations for sinusoidal excitations.

\subsection{Theory}

\subsubsection{The system description}

Our system consist of a drop of water sandwiched between two rigid glass plates. The glass plates were coated with a conducting layer and insulated with a hydrophobic dielectric layer of thickness $d$. The hydrophobic layer gives enough slip to the oscillating drop. The height of the sandwiched drop, i.e., the separation between the glass plates, is $H$ which is much smaller than the radius of the drop $R$. An AC voltage is applied to the conducting layers on the glass plates to drive the system. An illustration is shown in Fig. 7.1.

\subsubsection{Equations of motion}

To analyse the problem we start with the Navier Stokes equation (Eq. 1.1) in cylindrical coordinates. We assume that velocity $u_{z}$ in the $z$ direction of 


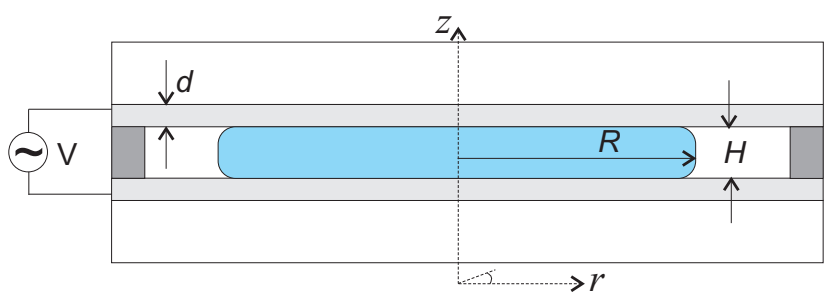

Figure 7.1: The illustration of the system. The values of the variables are given in the text.

the drop is zero. We also neglect gravity and inertial terms. The equations of motion are given by,

$$
\begin{array}{r}
\rho\left(\frac{\partial u_{r}}{\partial t}+u_{r} \frac{\partial u_{r}}{\partial r}+u_{\phi} \frac{\partial u_{r}}{r \partial \phi}-\frac{u_{\phi}^{2}}{r}\right)= \\
-\frac{\partial P}{\partial r}+\eta\left[\frac{\partial}{\partial r}\left(\frac{1}{r} \frac{\partial\left(r u_{r}\right)}{\partial r}\right)+\frac{\partial^{2} u_{r}}{r^{2} \partial \phi^{2}}+\frac{\partial^{2} u_{r}}{\partial z^{2}}-\frac{2}{r^{2}} \frac{\partial u_{\phi}}{\partial \phi}\right] \\
\rho\left(\frac{\partial u_{\phi}}{\partial t}+u_{r} \frac{\partial u_{\phi}}{\partial r}+u_{\phi} \frac{\partial u_{\phi}}{r \partial \phi}+\frac{u_{\phi} u_{r}}{r}\right)= \\
-\frac{\partial P}{r \partial \phi}+\eta\left[\frac{\partial}{\partial r}\left(\frac{1}{r} \frac{\partial\left(r u_{\phi}\right)}{\partial r}\right)+\frac{\partial^{2} u_{\phi}}{r^{2} \partial \phi^{2}}+\frac{\partial^{2} u_{\phi}}{\partial z^{2}}+\frac{2}{r^{2}} \frac{\partial u_{r}}{\partial \phi}\right] \\
\frac{\partial P}{\partial z}=0
\end{array}
$$

where $\rho$ is the density of the fluid, $\eta$ is it's viscosity and $p$ is the local pressure inside the drop. Assuming that the liquid is incompressible, we write the continuity equation as,

$$
\frac{\partial\left(r u_{r}\right)}{\partial r}+\frac{\partial u_{\phi}}{\partial \phi}=0
$$

The above equations are made dimensionless by setting $t=\tau t_{0}, u_{i}=$ $v_{i} V_{0}, r=s R, z=h H$, and $P=p P_{0}$ with $P_{0}=\gamma / R$ where $\gamma$ is the 
surface tension. This procedure results in the following equations when the terms with $(H / R)^{2}$ are neglected.

$$
\begin{gathered}
\frac{\partial v_{r}}{\partial \tau}=-\alpha \frac{\partial p}{\partial s}+\beta \frac{\partial^{2} v_{r}}{\partial h^{2}} \\
\frac{\partial v_{\phi}}{\partial \tau}=-\alpha \frac{\partial p}{s \partial \phi}+\beta \frac{\partial^{2} v_{\phi}}{\partial h^{2}} \\
\frac{\partial\left(s v_{r}\right)}{\partial s}+\frac{\partial v_{\phi}}{\partial \phi}=0
\end{gathered}
$$

where

$$
\alpha=\frac{t_{0} P_{0}}{\rho R V_{0}}=1
$$

and

$$
\beta=\frac{\eta t_{0}}{\rho H^{2}}
$$

Introducing the stream function ${ }^{1} \psi$, into the equations Eq. (7.5) to Eq. (7.7) and assuming $\psi(s, \phi, h)=f(h) \Psi(s, \phi)$ such that $\int_{0}^{1} f(h) d h=1$, where $\Psi$ is the height averaged stream function, we obtain

$$
\begin{gathered}
\left(\frac{\partial}{\partial \tau}+b\right) \frac{\partial \Psi}{s \partial \phi}=-\frac{\partial p}{\partial s} \\
\left(\frac{\partial}{\partial \tau}+b\right) \frac{\partial \Psi}{\partial s}=\frac{\partial p}{s \partial \phi}
\end{gathered}
$$

where, assuming parabolic flow profile, $b=-\beta \int_{0}^{1} f^{\prime \prime}(h) d h \simeq 12 \beta$.

\footnotetext{
$s v_{r}$

${ }^{1}$ In cylindrical coordinates, the stream function is defined as $\frac{\partial \psi}{\partial s}=-v_{\phi}$ and $\frac{\partial \psi}{\partial \phi}=$
} 


\subsubsection{Calculating the pressure, $p$}

The pressure inside the drop can be calculated by solving the Laplace equation in cylindrical coordinates,

$$
\nabla^{2} p=\frac{1}{s} \frac{\partial}{\partial s}\left(s \frac{\partial p}{\partial s}\right)+\frac{1}{s^{2}} \frac{\partial^{2} p}{\partial \phi^{2}}=0
$$

The solution of this equation can be obtained by separation of variables method. It gives infinite number of solutions,

$$
p(s, \phi)=p_{0}+\sum_{n=1}^{\infty}\left(p_{n} s^{n}+p_{n}^{\prime} s^{-n}\right) \cos \left(n \phi+\delta_{n}\right)
$$

The coefficient $p_{n}^{\prime}$ should be zero to prevent the pressure to diverge for $s \rightarrow 0$. Hence:

$$
p(s, \phi, \tau)=p_{0}+\sum_{n=1}^{\infty} p_{n}(\tau) s^{n} \cos \left(n \phi+\delta_{n}\right)
$$

where the coefficients $p_{n}$ and $\delta_{n}$ should be determined from the boundary conditions at $s=1$. The pressure at the boundary is related to the curvature via the Laplace pressure.

\subsubsection{The resonance frequencies}

The Eq. (7.14) is substituted in the equations of motion, Eq. (7.10) and Eq. (7.11). Integrating over $\phi$ and $s$ respectively, we obtain

$$
\begin{aligned}
& \left(\frac{\partial}{\partial \tau}+b\right) \Psi=-\sum_{n=1}^{\infty} p_{n}(\tau) s^{n} \sin \left(n \phi+\delta_{n}\right)+c(s, \tau) \\
& \left(\frac{\partial}{\partial \tau}+b\right) \Psi=-\sum_{n=1}^{\infty} p_{n}(\tau) s^{n} \sin \left(n \phi+\delta_{n}\right)+c(\phi, \tau)
\end{aligned}
$$

The above equations implies that $c(s, \tau)=c(\phi, \tau)=c(\tau)$. We assume $p_{n}(\tau)=A(\tau) p_{n}$ with $A(\tau)=\alpha e^{m \tau}$ and $m=j \Omega$. Assuming $\Psi=$ 
$A(\tau) H(s, \phi)$ and using separation of variables method to solve for $\Psi$, we get,

$$
\Psi(s, \phi, \tau)=-\left(\frac{\alpha}{m+b} \sum_{n=1}^{\infty} p_{n} s^{n} \sin \left(n \phi+\delta_{n}\right)+\frac{c}{m-b}\right) e^{m \tau}
$$

From the definition of the stream function, the velocity components along $r$ and $\phi$ direction can be calculated and integrating them with respect to $\tau$, the displacement fields $\xi_{r}$ and $\xi_{\phi}$ can be obtained as,

$$
\begin{aligned}
& \xi_{r}=\frac{-1}{m(m+b)} \sum_{n=1}^{\infty} n p_{n} s^{n-1} \cos \left(n \phi+\delta_{n}\right) e^{m \tau} \\
& \xi_{\phi}=\frac{1}{m(m+b)} \sum_{n=1}^{\infty} n p_{n} s^{n-1} \sin \left(n \phi+\delta_{n}\right) e^{m \tau}
\end{aligned}
$$

The position $r_{s}$ from the origin to any point on the boundary $(s=1)$ of the deformed drop can be determined from Eq. (7.18) and Eq. (7.19),

$$
r_{s}=\sqrt{\left(s+\xi_{r}\right)^{2}+\xi_{\phi}^{2}} \simeq 1+\xi_{r}
$$

From $r_{s}$, the local curvature and hence the Laplace pressure can be calculated. The first order curvature can be approximated as

$$
\begin{aligned}
K_{c} & =\frac{r_{s}^{2}+2\left(\partial_{\phi} r_{s}\right)^{2}-r_{s} \partial_{\phi}^{2} r_{s}}{\left(r_{s}^{2}+\left(\partial_{\phi} r_{s}\right)^{2}\right)^{3 / 2}}=1-\xi_{r}-\partial_{\phi}^{2} \xi_{r} \\
& =1+\frac{\left(1-n^{2}\right)}{m(m+b)} n p_{n} \cos \left(n \phi+\delta_{n}\right) e^{m \tau}
\end{aligned}
$$

The pressure at the boundary of the drop $p_{B}(\phi)=p_{0} K_{c}(\phi)$. Equating Eq. (7.22) and the $n^{\text {th }}$ term of Eq. (7.14) we obtain,

$$
m(m+b)=n\left(1-n^{2}\right)
$$

The dimensionless resonance frequency is,

$$
\Omega_{n}^{2}=n\left(n^{2}-1\right)-(b / 2)^{2}
$$


In the case of inviscid fluid approximation, $(b=0)$,

$$
\omega_{n}^{2}=\frac{\Omega_{n}^{2}}{t_{0}^{2}}=\frac{\gamma}{\rho R^{3}} n\left(n^{2}-1\right),
$$

is the (dimensional) natural frequencies of oscillation of the drop where $t_{0}^{2}=\rho R^{3} / \gamma$.

\subsubsection{The profile of the drop}

The profile of the drop is given by Eq. (7.20),

$$
r_{s}(\phi)=1+\xi_{r}(\phi)
$$

In the dimensional form,

$$
r(\phi)=R+C_{n} \sum_{n=1}^{\infty} \cos \left(n \phi+\delta_{n}\right)
$$

where $C_{n}=R \xi_{n}$ is the amplitude of oscillation of the drop from its equilibrium radius $R$. The coefficient $\xi_{n}$ is given by,

$$
\xi_{n}=n p_{n} /\left(\Omega^{2}-j \Omega b\right)
$$

The calculated profiles are given in Fig. 7.2. The mode $n=1$ is prevented by the incompressibility of the fluid and appear as a translation of the drop.

\subsubsection{The driven oscillations}

When driven by AC electowetting the drop oscillates with shape modes described by Eq. (7.27) as illustrated in Fig. 7.2. The resulting periodic change in the boundary of the drop can be written as an external pressure field at the boundary given by,

$$
p^{(e)}(\phi, \tau)=\sum_{n=1}^{\infty} p_{n}^{(e)} \cos (n \phi) e^{j \Omega \tau}
$$




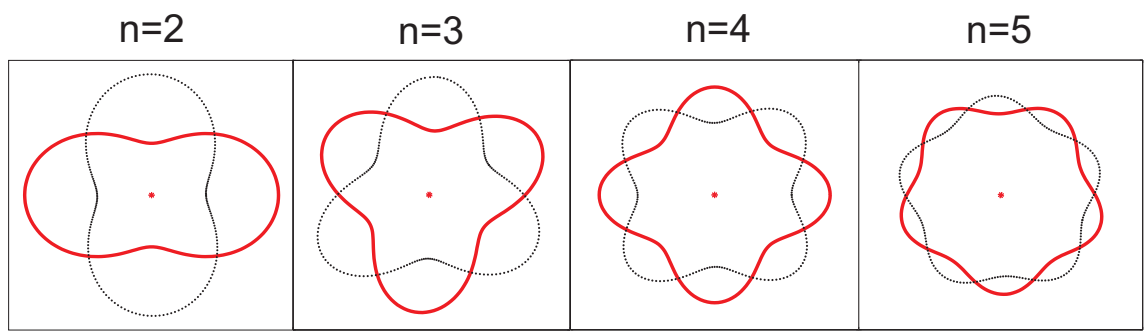

Figure 7.2: The calculated drop shapes at different eigen modes of oscillation, $n$. In each mode, the drop oscillates in time between the two shapes represented by dots and line.

Considering only the spatial dependent part $\Pi$ of Eq. (7.14) as

$$
\Pi(s, \phi)=\sum_{n=1}^{\infty} p_{n} \cos \left(n \phi+\delta_{n}\right)
$$

and applying the boundary condition using the identity in Eq. (7.21),

$$
\Pi(s=1, \phi)-\Pi^{(e)}(\phi)=-\xi_{r}(s=1, \phi)-\partial_{\phi}^{2} \xi_{r}(s=1, \phi),
$$

gives $\delta_{n}=0$ and we calculate

$$
p_{n}-p_{n}^{(e)}=\frac{\left(n^{2}-1\right)}{\left(\Omega^{2}-j \Omega b\right)} n p_{n}
$$

Substituting $p_{n}$ from Eq. (7.32) into Eq. (7.28), we get $\xi_{n}$. Finally, the amplitude of oscillations $C_{n}=R \xi_{n}$ can be written as

$$
\left|C_{n}\right|=R p_{n}^{(e)} T_{n}(\tilde{\omega})
$$

where

$$
T_{n}(\tilde{\omega})=\operatorname{Re}\left[\frac{\left(n^{2}-1\right)^{-1}}{\tilde{\omega}^{2}-j \tilde{\omega} \tilde{b}_{n}-1}\right]=\frac{\left(n^{2}-1\right)^{-1}}{\sqrt{\left(\tilde{\omega}^{2}-1\right)^{2}+\left(\tilde{\omega} \tilde{b}_{n}\right)^{2}}}
$$

with $\tilde{\omega}=\Omega / \Omega_{n}$ and $\tilde{b_{n}}=b / \Omega_{n}$ where $\Omega_{n}=n\left(n^{2}-1\right)$. From Eq. (7.33) it is clear that the amplitude of oscillations will show the properties of the transfer function $T_{n}(\tilde{\omega})$ (see Fig. 7.3). 


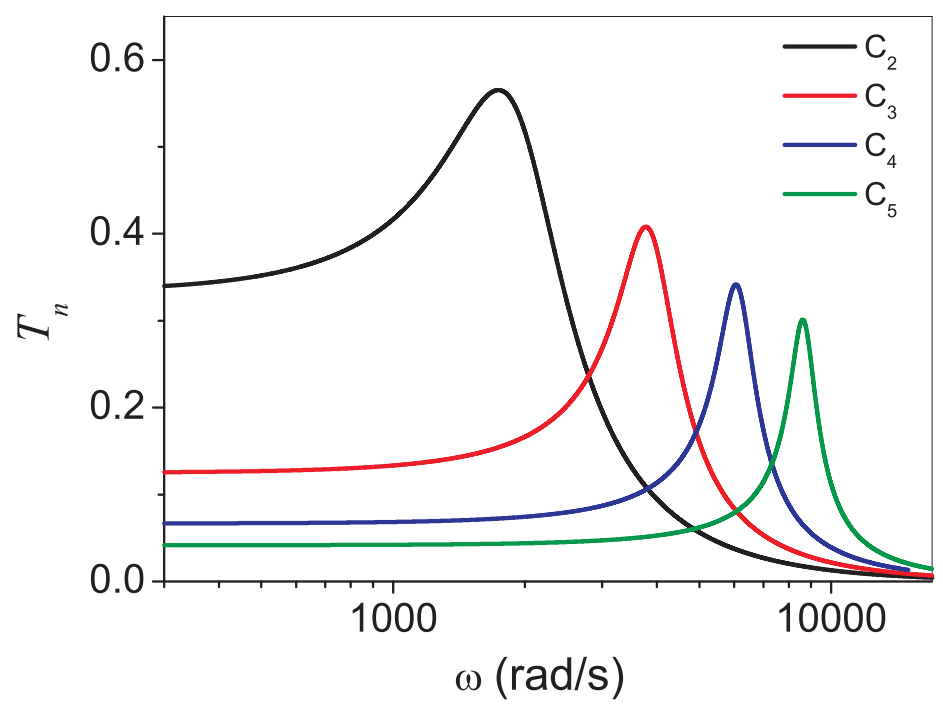

Figure 7.3: The function $T_{n}(\tilde{\omega})$ describing the amplitude behaviour of oscillations versus angular frequency. (Water drop with $\mathrm{R}=0.5 \mathrm{~mm}$ and $\mathrm{H}=100 \mu \mathrm{m}$.)

\subsection{Experiments}

For the experiments, we used glass substrates coated with conducting ITO layer and insulated with dielectric films of SU8 and Teflon of total thickness $d$ of $5 \mu \mathrm{m}$. The contact angle of water on the Teflon surface was $108^{\circ} \pm 2^{\circ}$ and the contact angle hysteresis ranged between $5^{\circ}$ to $15^{\circ}$. The height $\mathrm{H}$, ranges between 100 to $400 \mu \mathrm{m}$ was set by using spacer layers of parafilm. The liquid was $10 \mathrm{mM}(\mathrm{KCl})$ in DI water. An rms voltage of $150 \mathrm{~V}$ (resulting in an average contact angle of $80^{\circ}$ ) was applied to the substrate, while the applied frequency $f_{a p p}$ was swept from typically 10 $\mathrm{Hz}$ to $1000 \mathrm{~Hz}$ with a rate of $8.25 \mathrm{~Hz} / \mathrm{s}$. The shape oscillations of the drop were recorded using a CCD camera with a frame rate of $10 \mathrm{fr} / \mathrm{s}$. Moreover, the oscillations of the drop at a fixed frequency were recorded using a fast camera at a typical frame rate of $6000 \mathrm{fr} / \mathrm{s}$. 


\subsection{Results and discussion}

When the applied voltage was low, the drop did not oscillate. Increasing the voltage, the drop overcomes the pinning on the substrates and starts to oscillate. With increasing frequency, the drop gradually changes from mode $n=2$ to 6 . The mode $n=1$ is not oscillatory but only a translation over the substrate. The bottom view of the drop shapes at different modes of oscillations are shown in Fig. 7.4. The shape of the drop described by Eq. (7.27) is extracted from the measured images of the drop using home made Matlab code.
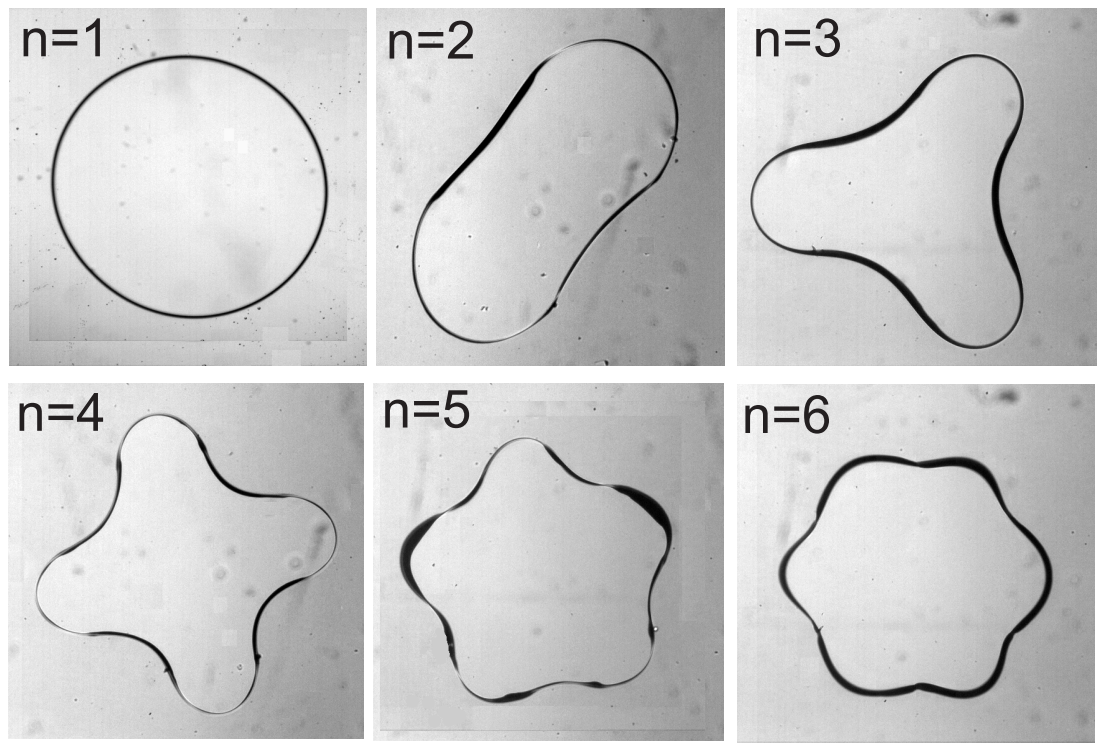

Figure 7.4: The measured drop shapes corresponding to different eigen modes, $n$. The first mode $(n=1)$ is only a translation. All other modes are oscillatory.

Considering the center of mass of the drop as origin, the equilibrium radius $R$ and the amplitude components $C_{n}$ are calculated as described in appendix B for drop shape at a given frequency. The amplitude of oscillations $C_{n}$ follow the transfer function Eq. (7.34). In Fig. 7.5, $C_{n} / R$ 


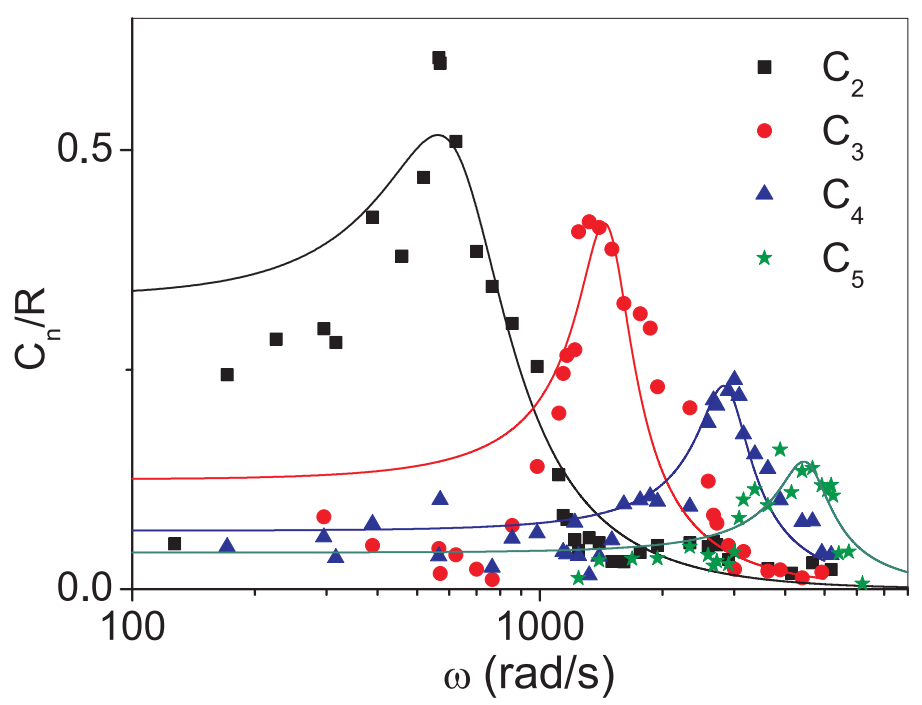

Figure 7.5: The amplitude of oscillations versus the angular frequency $\left(=2 \pi f_{a p p}\right)$ of the applied voltage for eigen modes $n=2,3,4$ and 5 . The line is a fit to the data. A maximum in the amplitude corresponds to the resonance frequency. Height and equilibrium radius of the drop were $100 \mu \mathrm{m}$ and $0.58 \mathrm{~mm}$ respectively. The contact angle hysteresis was about $5^{\circ}$.

is plotted as a function of the frequency which includes oscillation modes from two to five. For each mode $C_{n} / R$ is fitted with Eq. (7.34) with fitting parameters being $b$ and $\omega_{n}$. The peak of the curve corresponds to the eigen frequency $\omega_{n}$ of the oscillations. The parameter $b$ determines the width of the curves and is related to the viscous dissipation and height of the drop.

At resonance the drop shows oscillations with well defined shape corresponding to a particular mode. We determined the resonance frequency $\omega_{n}^{\text {exp }}$ from the bottom view images of the drop while gradually increasing the frequency of the applied voltage. The error in the $\omega_{n}^{\text {exp }}$ values was within $20 \%$. We observed that the $\omega_{n}^{\exp }$ values are considerably lower than the calculated values $\omega_{n}$ using Eq. (7.25). The measured resonance frequency of the drop was not consistent with it's size and varied from experiments to experiments. The ratio $\omega_{n}^{e x p} / \omega_{n}=\chi$ for different $n$ are 


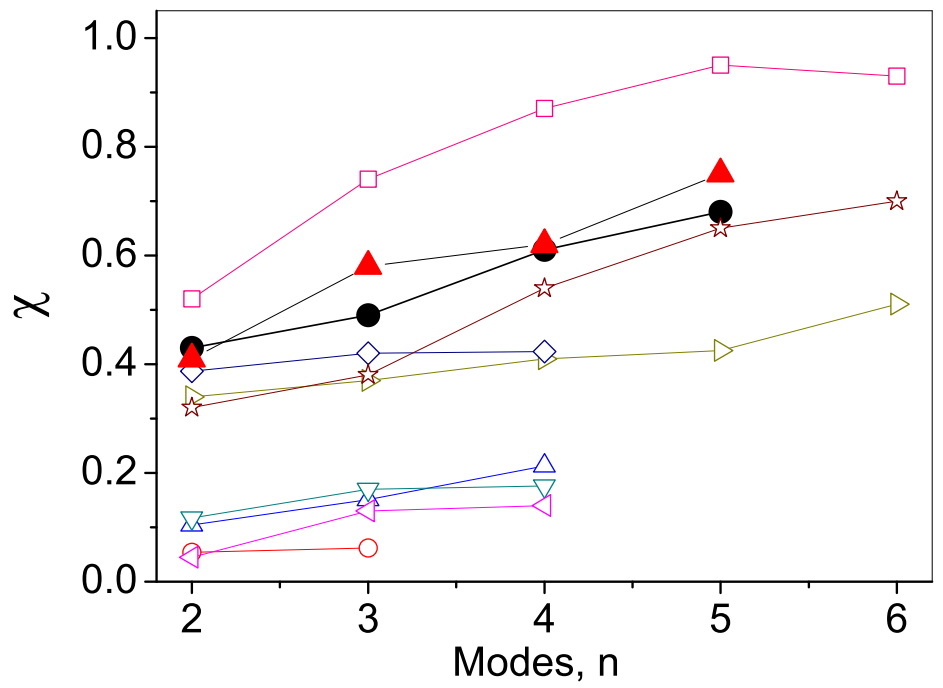

Figure 7.6: The ratio of measured and calculated eigen frequencies $\chi=\omega_{n}^{\exp } / \omega_{n}$ versus the eigen modes. Each symbol represents independent measurement on different substrates for different values of $\mathrm{H}$. No correlation between $\mathrm{H}$ and $\chi$ is observed. With large hysteresis $\left(\sim 15^{\circ}\right)$ the curves lie far down from the expected value, $\chi=1$. At lower modes $\omega_{n}^{e x p}$ deviates more from $\omega_{n}$.

shown in Fig. 7.6, for different experiments carried out. For each experiments different drops and substrates were used. The curve with filled circles corresponds to the data fitted in Fig. 7.4. It is clear that the measured resonance frequencies are substantially deviated from the expected values. Moreover, this deviation decreases with increasing the modes of oscillation, i.e. with increasing frequency. We used droplets with different height but the results in Fig. 7.6 did not show any correlation between the drop height and the deviation in the resonance frequencies. In fact when $R>>H$ the resonance frequency has only negligible influence on $\mathrm{H}$ especially at higher modes (see also Eq. (7.24)).

In order to investigate the dynamics of oscillations we recorded the 
drop shape evolutions with time while the sinusoidal applied frequency is fixed. The chosen frequency is such that the drop oscillates in one of the dominant shape modes. The oscillation amplitudes normalized by the equilibrium radius are given in Fig. 7.7 for $n=2,3,4$ and 5 for the applied frequencies at 80,220, 370 and $640 \mathrm{~Hz}$ respectively. Even though the drop shapes corresponds to a particular mode, it has contributions from all other infinite number of modes. These contributions are in fact negligible at resonance and shown in Fig. 7.7 upto $n=8$. The dominant amplitude of eigen mode 2,3,4 and 5 in Fig. 7.7A to D respectively, suggests that in each mode the drop oscillates at a frequency very close to its resonance. From Fig. 7.7, it is also clear that the amplitude of oscillations decreases with increasing $n$.

Moreover the oscillations are not sinusoidal as noticed for $n=2$ and 3 in Fig. 7.7A and B. The amplitude of oscillations increases sharply and stops for a while, then decreases back slowly. A fast Fourier transform (FFT) analysis reveals the small contributions from higher frequencies. With increasing modes the oscillations are close to sinusoidal and contributions from higher frequencies are negligible or cannot be detected (for Fig. 7.7C and D). Moreover $\chi$ for $n=2,3,4$ and 5 are $0.41,0.58,0.62$ and 0.75 respectively (filled triangle in Fig. 7.6). Some of our measurements produced oscillations identical to a square wave when $\chi \sim 0.1$. Fig. 7.6 and Fig. 7.7 reveal a correlation between non-sinusoidal oscillations and the deviations of the measured resonance frequencies of the drop from those calculated. 

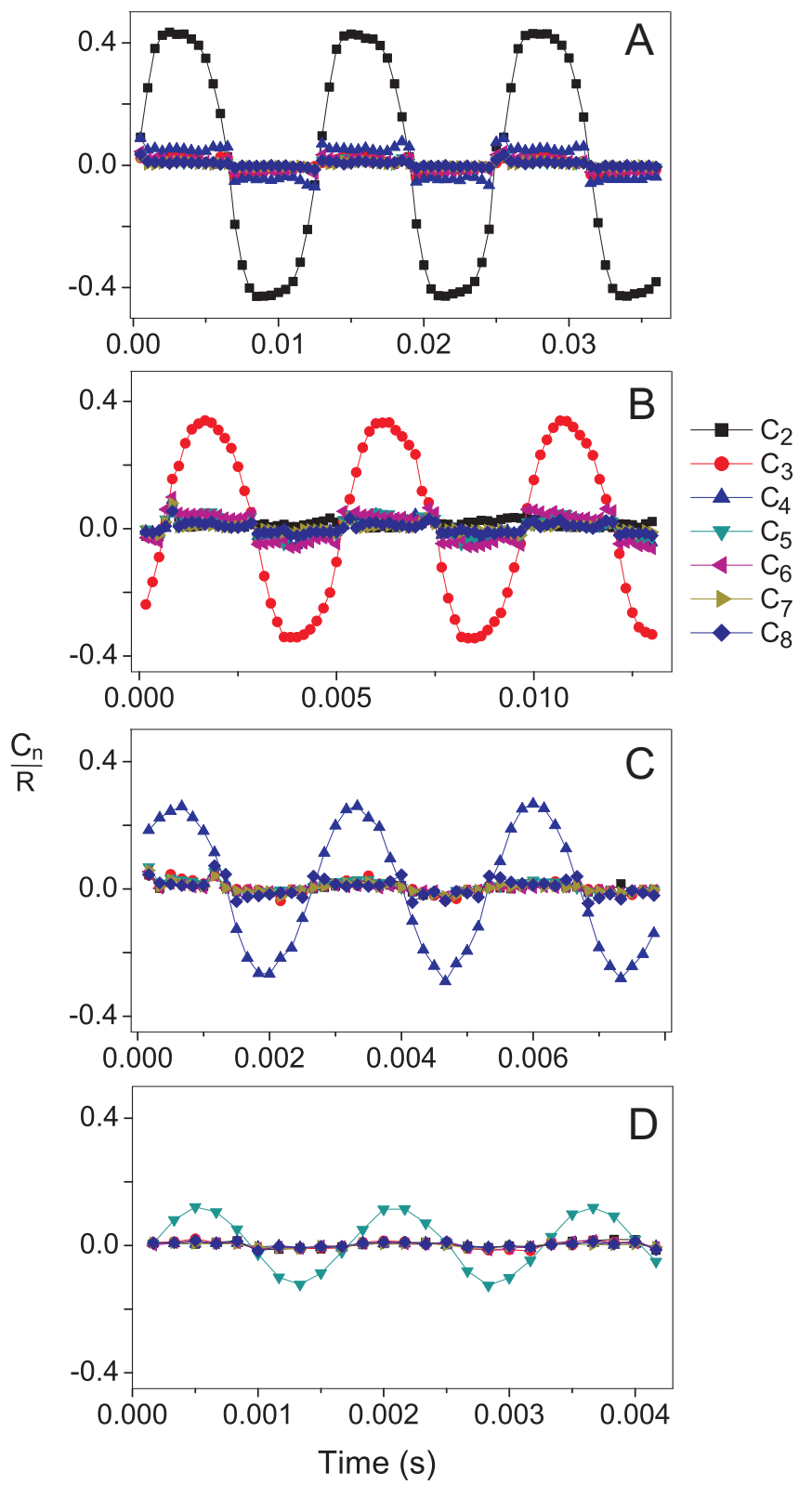

Figure 7.7: The amplitude of oscillations versus time for the dominating modes 2 to 5 in the panels A to D respectively. The frequency $f_{a p p}$ of the applied voltage was A) 80 $\mathrm{Hz}, \mathrm{B}) 220 \mathrm{~Hz}, \mathrm{C}) 370 \mathrm{~Hz}$ and D) $640 \mathrm{~Hz}$. The oscillations contain small contributions from all other modes. The contributions upto $n=8$ are shown in the figure. The oscillations are not exactly sinusoidal. 
We assume that the non-sinusoidal oscillations and the deviations in the resonance frequencies are caused by the pinning of the contact line on the substrate. The smoothness of the substrate is characterized by the contact angle hysteresis. When the hysteresis was large $\left(\sim 15^{\circ}\right)$ the resonances appeared at very low frequencies $(\chi \sim 0.1$ for $n=2)$ with very low amplitude. In contrast, very fresh samples with low hysteresis $\left(\sim 5^{\circ}\right)$ showed resonances at relatively higher frequencies $(\chi \sim 0.4$ for $n=2$ ) and with larger amplitudes. The hysteresis had an effect on the driving voltage also. When hysteresis was large, relatively larger voltage was required to begin the oscillations with a noticeable amplitude.

The hysteresis can strongly influence the eigen modes. Experiments on sessile drops showed, with increasing amplitude the resonance frequencies shifted towards lower values [148]. This complex behaviour was avoided by using hydrophobic substrates [116]. Fayzrakhmanova et al also reported a non-trivial shift of eigen modes of a sessile drop due to stick slip motion [111]. None of the above mentioned references shows such a substantial deviation in resonance frequencies as we observed experimentally. The experiments with flat droplets found in literature are carried out without sandwiching them between two substrates $[141,142,143,144,116,145,146,147]$ and no deviations in the resonance frequencies are reported. However a theoretical study on cylindrical drop sandwiched between two solid planes reports substantial reduction in the resonance frequencies with increasing hysteresis [149]. They showed that the resonance frequencies can be lowered by even a factor of two with increasing surface roughness.

The sandwiched droplet has two contact lines, i.e., on upper and lower substrates. This escalates the influence of hysteresis. Moreover, electrowetting bring the equilibrium contact angle close to the receding angle and away from the advancing angle. This makes the drop to recede easily but to advance slowly. Even a small amount of hysteresis further delays this advancing contact line. This implies that the oscillations are highly sensitive to the surface roughness. We assume that the effect of stick-slip motion due to surface roughness superimposes on the natural oscillations 
of the drop. If we compare the drop oscillation to a mass-spring oscillation, then the stick-slip dynamics may decrease the stiffness of the spring to a lower effective value. Consequently, this lowers the resonance frequencies. At lower modes where the contact line moves slowly, the stickslip effects are more pronounced than at higher modes where the contact line moves faster. This might be a reason why the measured natural frequencies are less deviated from those calculated ones at relatively higher modes (see Fig. 7.6).

In the theoretical formulations, we assumed that an external pressure field $p_{n}^{(e)}$, caused by electrowetting, drives the oscillations. In electrowetting the actual driving frequency is twice the applied frequency from the power supply (see chapter 1). Our measurements show that the drop oscillates with a frequency, half of the driving frequency. This is a characteristic behaviour for parametrically excited oscillations. Although we do not understand the actual force driving the oscillations, the above mentioned observation points to a possible parametric forcing. AC electrowetting periodically changes the radius of the drop. This may result in the periodic modulations in the resonance frequency of the drop inducing parametric forcing.

\subsection{Conclusion}

In conclusion, we have shown that a sandwiched drop between two hydrophobic glass plates can be oscillated in different shape modes by electrowetting. The drop oscillates with half of the driving frequency. Moreover, the oscillations are not exactly sinusoidal. The eigen modes appear at lower frequencies than expected. A further understanding of the influence of hysteresis is essential to explain the shift in resonance frequencies. In addition, further theoretical investigations must be carried out to perceive the actual driving mechanism of the oscillations. 


\section{Acknowledgements}

We acknowledge M.H.P. van der Weide-Grevelink for providing data for some of the curves in Fig. 7.6. 


\section{Chapter 8}

\section{Conclusion and outlook}

This thesis describes methods to control fluid flow produced in microchannels and minute drops by the application of electric fields. In microchannels, we control electro osmotic flow using gate electrodes embedded on the channel walls. On the other hand, in sessile drops, we generate flows by electrowetting technique and control those flow patterns using pinning sites at the contact line. Moreover, oscillations of discrete drops bound between two solid substrates are studied. In the following sections of this chapter, the conclusion and the outlook of the studies are presented. 


\subsection{Conclusion}

We have developed a method to determine the surface charge in microchannels in situ, using the solution displacement method. We have presented a simple analytical model to evaluate the time dependence of the measured current during the displacement process. From the obtained displacement time we calculate the surface charge as well as the zeta potential and the electroosmotic flow velocity at given electrolyte concentrations. Although diffusion effects near the interface between the displacing solutions are neglected, the model is applicable for a large range of concentration ratios, because it does take the pressure variation inside the channel into account. The deviations in the current versus time dependence near the transition to the plateau are quite large for concentration ratios larger than four. However, the displacement time (and hence the surface charge) can be well determined from the short time dependence of the current and is hardly sensitive to these deviations. Hence, for strong 1-1 electrolytes, the proposed method is adequate to determine the surface charge in microchannels in a simple way.

We have shown that electro osmotic shear flow can be created by using chemical modification of the surface charge. The adsorption of chemicals by the opposite bare wall can destroy the shear flow. Therefore this technique is limited by the amount of chemical coating that can present on one of the channel walls. This limitation can be overcome by using gate voltage to change the zeta potential of the channel wall. The $\mathrm{SiO}_{2}$ dielectric layer deposited on the gate electrodes by using PECVD technique, has very low negative surface charge compared to glass or PDMS. This property also can be used to create shear flow. By modulating the surface charge of this $\mathrm{SiO}_{2}$ layer with gate voltage, shear rate is increased by $42 \%$. By increasing the strength of electric field along the axis of the channel, higher shear rates can be produced.

Our experiments on sessile drops show that AC electrowetting drives oscillations at the air-liquid interface. Consequently, internal drift flow patterns can be generated, not only in the vertical plane but also in the azimuthal plane, parallel to the substrate. By using artificial pinning sites, 
one can control the vortex patterns in the azimuthal plane. We suppose that the azimuthal flow patterns originate from the broken symmetry of stress oscillations at the three phase contact line. The suppression of stress oscillations at the pinning sites results locally in a different amplitude of the contact line motion. As a result, an aperiodic drift motion superimposes the periodic oscillations of the liquid. This drift forms the flow vortices over many oscillation cycles. The frequency dependence of the flow strength in these vortices is described by the resonance frequencies of the corresponding droplet surface oscillations.

By sweeping the frequency of the applied voltage in an electrowetting sessile drop with locally pinned contact line, the internal flow vortices can be moved towards the pinning site at the contact line. The moving flow vortices accumulate solute particles near the contact line. This technique can be used to pre-concentrate particles or bio-molecules locally inside a sessile droplet.

The internal flow patterns and the contact line oscillations of electrowetting sessile drops can be applied to suppress the coffee stain effect. Coffee stains of both colloidal particles of various sizes and DNA solutions can be suppressed at alternating frequencies ranging from a few $\mathrm{Hz}$ to a few tens of $\mathrm{kHz}$. The success in suppressing the coffee stain effect by electrowetting depends on the particle size, their initial concentration inside the drop and the strength of the applied voltage.

We have shown that a sandwiched drop between two hydrophobic glass plates can be oscillated in various shape modes by electrowetting. The drop oscillates with half of the driving frequency. Moreover, the oscillations are not pure sinusoidal. The amplitude of oscillations follows the theory. However, the eigen modes appear at lower frequencies than those expected from the theory. 


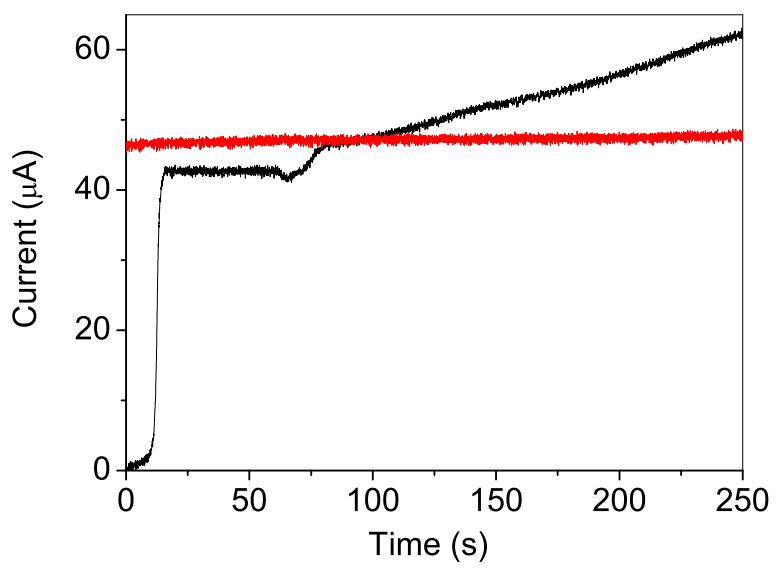

Figure 8.1: Current versus time plot (see also chapter 3) for $100 \mathrm{mM} \mathrm{KCl}$ displacing $1 \mathrm{mM} \mathrm{KCl}$ (black curve). The displacement takes place within about $15 \mathrm{~s}$ where the current reaches a plateau value. However, there is an unexpected increase in the current at a later time, at about $65 \mathrm{~s}$. Measurements for very long time duration revealed (data not shown) that the current drops to a value close to the red curve. The red curve is the expected plateau value of the current obtained when the channel and reservoirs are filled with $100 \mathrm{mM}$.

\subsection{Outlook}

The theoretical model to determine the surface charge of the microfluidic channels does not consider diffusion at the interface between the two displacing solutions. The model can be improved by including the diffusion effects at the interface. Moreover, we have observed that the current versus time curves have an abnormal behaviour when measured at low $\mathrm{pH}$ of the electrolyte. An unexpected decrease of the current occurs followed by the expected increase as the high conductivity solution fills the channel.

When two solutions of large conductivity difference are displaced in the channel, we observe an unexpected increase in the current after the completion of the displacement process (Fig. 8.1). These observations are 


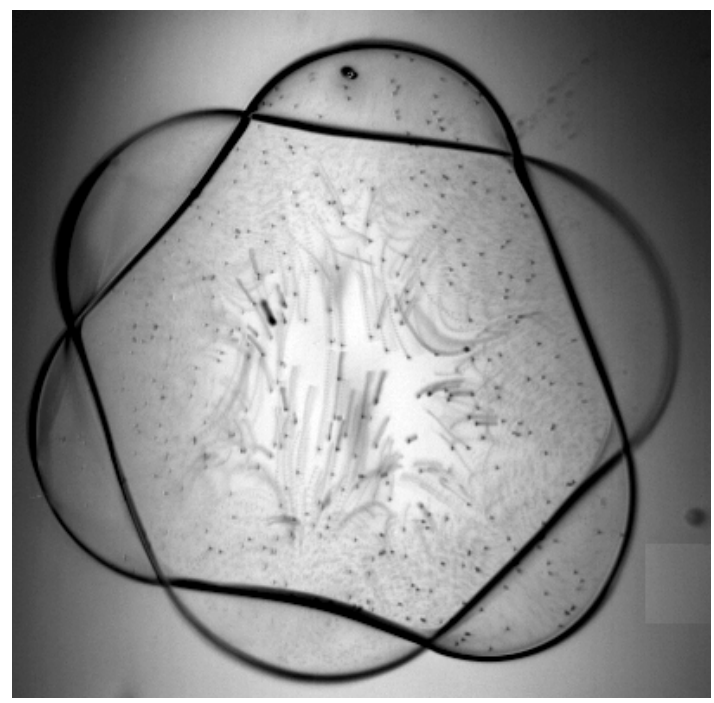

Figure 8.2: A stroboscopic image of oscillations (in mode three at $170 \mathrm{~Hz}$ ) of a sandwiched drop obtained by stacking the consecutive frames of the movie. The drop oscillates in time between the two overlaid three-lobe shapes. The tracer particles oscillate along with the liquid. However, they show a net displacement characterised by the dark trajectories inside the drop. This implies a net displacement of the liquid which can be used for mixing in sandwiched drops.

not well understood. They must be arising from some chemical processes going on in the channel. Using buffer solutions as electrolytes such effects must be investigated further.

To improve the performance of gate electrodes, better dielectric layers must be used over it. The PECVD grown layers are found to breakdown easily. Dielectric layers of aluminium oxide or LPCVD grown silicon nitride are a solution to this issue.

The internal flow and anticipated enhanced mixing efficiency have potential applications, in stirring and mixing on the $\mu \mathrm{l}$ scale or suppressing the settling of (micro) particles in drying drops, e.g. the coffee stain effect. To control the flow patterns we used pinning sites at the contact line. It is also possible to make pinning sites beneath the dielectric layer as elec- 
trodes and their pinning strength can be tuned by applying DC voltage. Using multiple electrodes, the number of pinning sites can be changed dynamically, improving the mixing efficiency.

Adsorption is a bottleneck when electrowetting is performed with biological liquids. This adsorption occurs due to the hydrophobic interactions. Decreasing adsorption will improve the efficiency of applications like sample pre-concentration or suppression of coffee stains of biological samples.

As we have seen in Chapter 7, a further understanding of the influence of surface roughness is essential to explain the oscillation behaviour of sandwiched drops. In addition, further theoretical investigations must be carried out to perceive the actual driving mechanism of these oscillations. The sandwiched drop oscillations can be used for mixing in droplet based microfluidics. The oscillations of the drop create net internal flows (see Fig. 8.2) due to slight asymmetries in the oscillations. Chaotic convections can be created in the droplet by driving it in different oscillation modes by changing the frequency of the applied voltage in a random manner. 


\section{Appendix A}

\section{Surface charge - pH -ionic concentration}

Due to the intrinsic buffer capacity of a surface (e.g. silica), a change in bulk $\mathrm{pH}$ from $\mathrm{pH}_{z c}$ causes the surface to titrate to get back into equilibrium. For example, when the bulk $\mathrm{pH}$ increases from $\mathrm{pH}_{z c}, \mathrm{SiO}_{2}$ surface dissociates to produce more protons near the surface to keep the $\mathrm{pH}$ there close to $\mathrm{pH}_{z c}$. This causes the surface left with a negative charge. The intrinsic buffer capacity of the surface, $\beta_{\text {int }}$ can be defined as

$$
\frac{d \sigma_{0}}{d p H_{s}}=-F \beta_{\text {int }}
$$

where $\sigma_{0}$ is the surface charge density, $p H_{s}$ is the surface $\mathrm{pH}$ and $F$ is the Faraday's constant. The intrinsic buffer capacity cannot be measured directly since the $\mathrm{pH}$ of the surface differs from that of the bulk. The bulk region is separated from the surface by the Debye layer. Following the formulations by Eijkel et al [6], we get an expression describing how the surface charge density changes with the bulk $\mathrm{pH}$,

$$
\frac{d \sigma_{0}}{d p H_{b}}=\left[\frac{-1}{F \beta_{i n t}}+\frac{F}{2.3 R_{g} T C_{D}}\right]^{-1}
$$

The above equation gives the surface charge as a function of the bulk $\mathrm{pH}$. The term $C_{D}$, which is the diffuse layer capacitance, depends on the ionic 
concentration of the electrolyte. Therefore surface charge density also depends on the ionic concentration of the electrolyte.

The solution of Poisson-Boltzmann equation describing the potential at the double layer requires a choice of boundary conditions, usually either constant charge (CC) or the constant potential (CP) condition is used. The assumption of $\mathrm{CC}$ is realistic for systems where the surface charge originates either from strong acid or base groups or from isotopic substitutions of minerals (e.g., mica). CP condition is a good approximation for metal oxide surfaces at low ionic strengths and near its point of zero charge. Force measurements in the diffuse layer have shown that in reality, neither the $\mathrm{CC}$ nor the $\mathrm{CP}$ is a good approximation [150]. Both these effects can occur in a real system and is referred to as charge regulation.

\section{Gate controlling the zeta potential}

When $F \beta_{\text {int }} \gg 2.3 R_{g} T C_{D} / F$, the surface is said to have high intrinsic buffer capacity. The intrinsic buffer capacity of a substrate is undesirable during gate control of the zeta potential. Instead of changing the surface potential, it actually changes the surface charge by association or dissociation of the surface groups.

In an electrical analogue (Fig. A.1), we can define a capacitance to the surface, due to its intrinsic buffer capacity which is in series with the diffuse layer capacitance, $C_{D}$. The voltage applied on the gate electrode is mostly dropped on the dielectric layer over it. The capacitance of it determines the charge flowing through the surface and the liquid. This charge is distributed over the buffer capacitance of the surface, $F^{2} \beta_{\text {int }} / 2.3 R_{g} T$ and the diffuse layer capacitance $C_{D}$. The amount of charge stored depends on the capacitance. When the buffer capacitance is the largest, most of the charge is stored in it as dissociated protons. This results the zeta potential to stay unchanged. For silicon oxide $F^{2} \beta_{\text {int }} / 2.3 R_{g} T \gg C_{D}$ above $\mathrm{pH} \sim 4$ and therefore, gate modification of zeta potential is less effective 


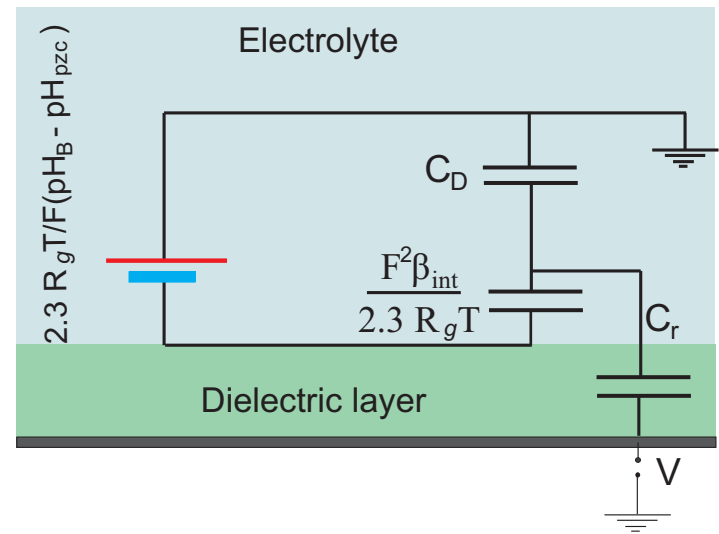

Figure A.1: The electric equivalent diagram of a double layer actuated with voltage on the gate electrode. The difference between bulk $\mathrm{pH}$ and $\mathrm{pH}_{z c}$ of the oxide is represented as a voltage source charging the oxide (the intrinsic surface charge). When a voltage is applied on the gate electrode, the charge flowing through the insulator (determined by $\mathrm{C}_{r}$ ) is distributed over the diffuse layer capacitor $\mathrm{C}_{D}$ and the buffer capacitor $F^{2} \beta_{\text {int }} / 2.3 R_{g} T$ whereby largest charge flows through the largest capacitor. On the buffer capacitor, charge is stored as protons and on $\mathrm{C}_{D}$ as double layer cations.

above this $\mathrm{pH}$. 



\section{Appendix B}

\section{Calculation of the oscillation amplitude}

A general expression for the radius of a sandwiched drop oscillating at frequency $\omega$ is given by

$$
r(\phi, t)=R+\sum_{n=1}^{\infty}\left[A_{n} \cos (n \phi)+B_{n} \sin (n \phi)\right] \cos (\omega t)
$$

where $R$ is the equilibrium radius, $\phi$ is the azimuthal angle (see Fig. B.1). The coefficients $A_{n}$ and $B_{n}$ can be determined using the orthogonality conditions,

$$
\begin{aligned}
& \int_{0}^{2 \pi} r(\phi) \cos (l \phi)=\delta_{l n} A_{n} \pi \\
& \int_{0}^{2 \pi} r(\phi) \sin (l \phi)=\delta_{l n} B_{n} \pi
\end{aligned}
$$

where $\delta_{l n}$ is the Kronecker delta function. The coefficients are given by

$$
\begin{aligned}
& A_{n}=\frac{1}{\pi} \int_{0}^{2 \pi} r(\phi) \cos (n \phi) d \phi \\
& B_{n}=\frac{1}{\pi} \int_{0}^{2 \pi} r(\phi) \sin (n \phi) d \phi
\end{aligned}
$$




$$
R=\frac{1}{2 \pi} \int_{0}^{2 \pi} r(\phi) d \phi
$$

The amplitude of oscillation of the mode $n$ is given by $C_{n}=\sqrt{A_{n}^{2}+B_{n}^{2}}$.

In order to calculate the coefficients $A_{n}, B_{n}$ and $R$ from the set of measured data points $\left(x_{i}, y_{i}\right)$ of the boundary of the drop, we start with finding the center of mass coordinates of the drop. The coordinates can be calculated numerically as

$$
\begin{aligned}
& x_{c m}=\frac{1}{D} \sum_{i=1}^{N-1} x_{i} y_{i}\left(x_{i+1}-x_{i}\right) \\
& y_{c m}=\frac{1}{2 D} \sum_{i=1}^{N-1} y_{i}^{2}\left(x_{i+1}-x_{i}\right)
\end{aligned}
$$

with

$$
D=\sum_{i=1}^{N-1} \frac{y_{i}+y_{i+1}}{2}\left(x_{i+1}-x_{i}\right)
$$

where $i=1,2,3 \ldots N$ with $i=1$ and $i=N$ are being the same data points. $A_{1}$ and $B_{1}$ are used to correct $\left(x_{c m}, y_{c m}\right)$. By taking $\left(x_{c m}, y_{c m}\right)$ as origin, the coordinates $\left(\phi_{i}, r_{i}\right)$ can be calculated as

$$
\begin{gathered}
r_{i}=\sqrt{\left(x_{i}-x_{c m}\right)^{2}+\left(y_{i}-y_{c m}\right)^{2}} \\
\phi_{i}=\arctan \left(\left(y_{i}-y_{c m}\right) /\left(x_{i}-x_{c m}\right)\right)
\end{gathered}
$$

In order to evaluate Eq.(B.4) to Eq.(B.6), the $N$ number of data points $\left(\phi_{i}, r_{i}\right)$ describing the shape of the drop are numerically integrated as below,

$$
A_{n}=\frac{1}{\pi} \int_{0}^{2 \pi} r(\phi) \cos (n \phi) d \phi=\frac{1}{\pi} \sum_{i=1}^{N-1} \int_{\phi_{i}}^{\phi_{i+1}}\left(r_{i+1 / 2}+\phi r_{i+1 / 2}^{\prime}\right) \cos (n \phi) d \phi
$$


which can be expanded as,

$\frac{1}{\pi} \sum_{i} \frac{1}{2}\left(r_{i}+r_{i+1}\right) \int_{\phi_{i}}^{\phi_{i+1}} \cos (n \phi) d \phi+\frac{1}{\pi} \sum_{i}\left(\frac{r_{i+1}-r_{i}}{\phi_{i+1}-\phi_{i}}\right) \int_{\phi_{i}}^{\phi_{i+1}} \phi \cos (n \phi) d \phi$

In a similar way $R$ and $B_{n}$ are determined for the different modes, $n$. Substituting the above determined $A_{n}, B_{n}$ and $R$ from the experimental data, into Eq.(B.1), the measured profile can be reproduced. An example is given in Fig. B.1.

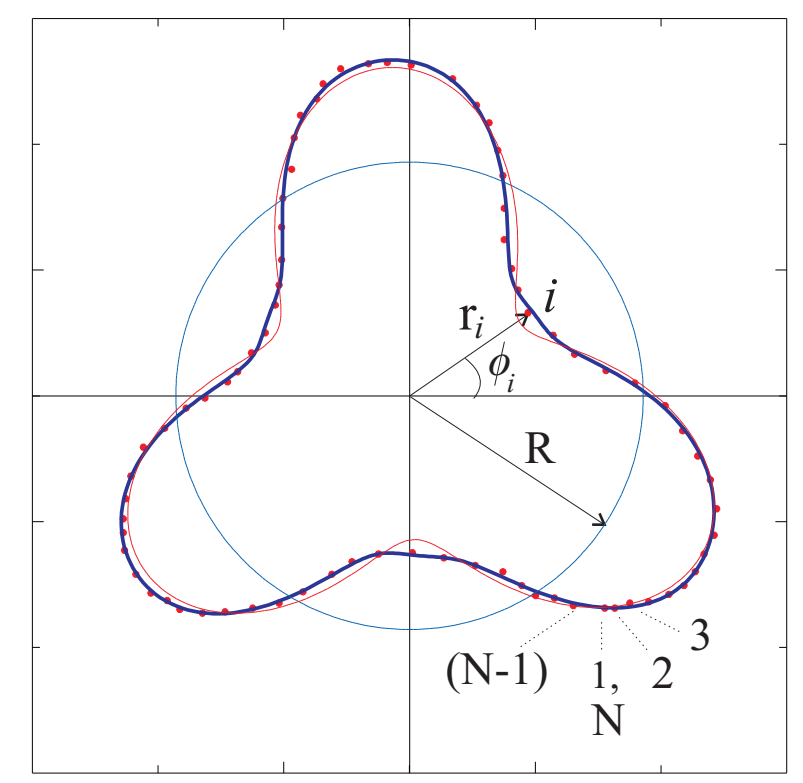

Figure B.1: Boundary of a drop oscillating in $n=3$ mode. The red filled circles are the measured data points. They are numbered as $i=1,2,3 \ldots N$ where 1 and $N$ represent the same data point. The curves are the calculated boundary by using $A_{n}, B_{n}$ and $R$ obtained from the measured data points as described in the text. They are for $\mathrm{n}=0$ (circle), $\mathrm{n}=0,1,2,3$ (red line) and $\mathrm{n}=0,1,2, . ., 10$ (thick blue line). 



\section{Appendix C}

\section{List of symbols}

\section{Roman symbols}

\begin{tabular}{lll} 
Symbol & Meaning & Expression \\
\hline \hline & & \\
$a, a_{n}$ & Activity coefficient & \\
$b_{s}$ & Slip length & \\
$c_{i}$ & Molar concentration of ion $i$ & \\
$c_{0}$ & Standard molarity of 1 mol/L & \\
$C_{D}$ & Diffuse layer capacitance & \\
$C_{w}$ & Wall capacitance & \\
$d$ & Thickness of dielectric layer & \\
$D$ & Typical length scale & \\
$e$ & Fundamental charge & \\
$E$ & Electric field & \\
$f_{i}$ & Inertial force & \\
$f_{v}$ & Viscous force & \\
$f$ & Body force per volume & \\
$f_{a p p}$ & Frequency of applied voltage & \\
$F$ & Faraday constant & \\
$F_{e x}$ & Horizontal part of the electrostatic force &
\end{tabular}


$h \quad$ Height of the channel

I Electric current

$k_{B} \quad$ Boltzmann constant

$1.38 \times 10^{-23} \mathrm{~J} / \mathrm{K}$

$K_{c} \quad$ Curvature of an interface

$\kappa \quad$ Debye-Hückel parameter

$\left(e^{2} \sum_{i} n_{i}^{\infty} Z_{i}^{2} / \varepsilon_{0} \varepsilon_{l} k_{B} T\right)^{1 / 2}$

L Channel length

$n_{i} \quad$ Number concentration of ion $i$

$n_{i}^{\infty} \quad$ Bulk number concentration of ion $i$

$N_{A} \quad$ Avagadro constant

$N_{e w} \quad$ Electrowetting number

$6.022 \times 10^{23} \mathrm{~mol}^{-1}$

$p H_{z c} \quad$ pH of zero charge

$P \quad$ Pressure

$R_{g} \quad$ Gas constant

$\varepsilon_{0} \varepsilon_{r} V^{2} / 2 d \gamma$

$R_{0} \quad$ Initial base radius of a sessile drop

Re Reynolds number

$R_{\Omega} \quad$ Electric resistance

$t \quad$ Time

$T \quad$ Temperature

$\underline{u} \quad$ Velocity field

$u \quad$ Fluid velocity

$u_{e o} \quad$ EOF velocity

$V \quad$ Potential

W Channel width

$x, y, z \quad$ Cartesian coordinates

$Z_{i} \quad$ Valance of ion $i$ 


\section{Greek symbols}

\begin{tabular}{lll} 
Symbol & Meaning & Expression \\
\hline \hline & & \\
$\gamma$ & Air-water interface tension & $73 \mathrm{mN} / \mathrm{m}$ at $20^{\circ} \mathrm{C}$ \\
$\gamma_{s g}$ & Solid-gas interface tension & \\
$\gamma_{s l}$ & Solid-liquid interface tension & \\
$\varepsilon_{0}$ & Permittivity of free space & $8.85 \times 10^{-12} \mathrm{coul}^{2} / \mathrm{Nm}^{2}$ \\
$\varepsilon_{r}$ & Dielectric constant of the insulating layer & \\
$\varepsilon_{l}$ & Dielectric constant of the liquid & \\
$\eta$ & Dynamic viscosity of liquid & \\
$\theta_{Y}$ & Young's contact angle & \\
$\theta$ & Apparent contact angle & \\
$\lambda_{D}$ & Debye length & \\
$\mu_{e c}$ & Electrochemical potential & \\
$\mu_{i}$ & Chemical potential of ion $i$ & \\
$\mu_{i}^{0}$ & Standard chemical potential of ion $i$ & \\
$\rho$ & Density of liquid & \\
$\sigma$ & Surface charge density & \\
$\sigma_{m}$ & Marangoni stress & \\
$\phi$ & Azimuthal angle & \\
$\psi_{\zeta}$ & Zeta potential
\end{tabular}

\section{Chapter 3}

\begin{tabular}{lll} 
Symbol & Meaning & Expression \\
\hline \hline & & \\
$C$ & Ionic concentration & $\mathrm{mol} / \mathrm{L}$ \\
$C_{\text {init }}, C_{f i n}$ & Initial and final ionic concentration & \\
$c$ & Ratio $i$ & $C_{\text {init }} / C_{f i n}$ \\
$g, g_{r}$ & Correction parameters & \\
$I_{0}$ & Initial current &
\end{tabular}




\begin{tabular}{ll} 
Symbol Meaning Expression \\
\hline \hline
\end{tabular}

$\begin{array}{lll}I_{\infty} & \text { Plateau current } & \\ I_{\text {min }} I_{\text {max }} & \text { Fitting range for current } & \\ \mathrm{p} & \text { Pressure } & \\ \mathrm{P}, \mathrm{Q} & \text { Constant } & 1-c, 1-c^{3 / 2} g_{r} \\ t_{0} & \text { Characteristic time that depends on } C_{\text {init }} & L / u_{e o}\left(C_{\text {init }}\right) \\ t_{d} & \text { Displacement time } & t(\lambda=1) \\ t_{\text {shift }} & \text { Time shift as a correction parameter } & \\ \beta & & \\ \beta & \text { Constant } & L_{1} / L \\ \lambda & \text { Ratio of lengths } & \\ \mu_{n} & \text { Mobility of ionic species } n & \\ \sigma_{P B} & \text { Surface charge with full PB solution } & \end{array}$

\section{Chapter 4}

\begin{tabular}{lll} 
Symbol & Meaning & Expression \\
\hline \hline & & \\
$c_{e}$ & Constant & $-\varepsilon_{0} \varepsilon_{l} E_{\infty} W h / 2 \eta$ \\
$c_{p}$ & Constant & $W h^{3} / 12 \eta$ \\
$E_{\infty}$ & Electric field across the channel & \\
$k$ & $k^{t h}$ region of the channel & \\
$l, l^{\prime}, l^{\prime \prime}$ & Length scales \\
$m$ & Electro osmotic flow mobility & \\
$\mathrm{N}$ & Total number of gate electrodes & \\
$\dot{Q}$ & Volumetric flow rate & \\
$U$ & $V_{G}$ independent of $z$ (by the resistive network) & \\
$V_{n}$ & Potential on a gate electrode & \\
$V_{t o t}$ & Total applied potential to the resistive network &
\end{tabular}


Symbol Meaning Expression

$V_{\infty} \quad$ Applied potential across the channel

$V_{G} \quad$ Potential $\quad V_{n}-\varphi_{\infty}(z)$

w Width of a gate electrode

$v_{ \pm} \quad$ Velocity of ions

$\pm \quad$ Positive or negative ion

$\dot{\gamma} \quad$ Shear rate

$\zeta_{ \pm} \quad$ Friction coefficient of ions

$\varphi \quad$ Total potential in the channel $\quad \psi(y, z)-z E_{\infty}$

$\varphi_{\infty}(z) \quad$ Potential in the channel due ti $E_{\infty} \quad-z E_{\infty}$

$\psi \quad$ Potential relative to that in the bulk

$\psi_{a}, \psi_{b} \quad \psi$ at lower and upper walls

$\psi_{s} \quad \psi$ when the gate voltage is applied

$\tau \quad$ Shear stress

\section{Chapter 5}

Symbol Meaning Expression

$f_{n} \quad$ Calculated resonance frequency

$f_{P} \quad$ Frequency corresponding to flow velocity peaks

$L_{v c} \quad$ Distance between vortex center and pinning site

$q \quad$ Wave number of capillary waves

$\omega \quad$ Angular frequency of capillary waves 


\section{Chapter 6}

\begin{tabular}{lll} 
Symbol Meaning & Expression \\
\hline \hline
\end{tabular}

$A_{T C L} \quad$ Amplitude of oscillation of triple contact line

$f_{d r v} \quad$ Driving frequency $2 f_{\text {app }}$

$R_{f} \quad$ Radius of stains after evaporation

$V_{\text {sat }} \quad$ Voltage at which the contact angle saturates

$V_{t h} \quad$ Threshold voltage

$\phi_{c} \quad$ Initial volume concentration of particles in the drop

\section{Chapter 7}

\begin{tabular}{lll} 
Symbol & Meaning & Expression \\
\hline \hline & & \\
$b$ & Constant & $\simeq 12 \eta t_{0} / \rho H^{2}$ \\
$C_{n}$ & Amplitude of oscillations & $R \xi_{n}$ \\
$h$ & Dimensionless height & $z / H$ \\
$H$ & Drop height & $\sqrt{\gamma / \rho g}$ \\
$l_{c}$ & Capillary length & $j=\sqrt{-1}$ \\
$m$ & $j \Omega$ & $\mathrm{n}=1,2 \ldots \infty$ \\
$n$ & Modes of oscillation & $P / P_{0}$ \\
$p$ & Dimensionless pressure & \\
$p^{e}$ & Dimensionless external pressure field & \\
$P_{0}$ & Laplace pressure & $\gamma / K_{c}$ \\
$r$ & Radius of the oscillating drop & \\
$R$ & Equilibrium radius of the drop & $r / R$ \\
$s$ & Dimensionless radius & \\
$T_{n}$ & A function of $\omega$ & $u / V_{0}$ \\
$v$ & Dimensionless velocity &
\end{tabular}




\begin{tabular}{lll} 
Symbol & Meaning & Expression \\
\hline \hline & & \\
$\alpha, \beta$ & Constants & $t_{0} P_{0} / \rho R V_{0}, \eta t_{0} / \rho H^{2}$ \\
$\delta$ & Phase shift & \\
$\xi_{r} \xi_{\phi}$ & $r$ and $\phi$ components of displacement & \\
$\xi_{n}$ & Dimensionless amplitude of oscillation & $n p_{n} /\left(\Omega^{2}-j \Omega b\right)$ \\
$\Pi$ & Spatial part of $p^{e}$ & \\
$\tau$ & Dimensionless time & $t / t_{0}$ \\
$\psi$ & Stream function & \\
$\Psi$ & Height averaged stream function & \\
$\omega$ & Angular frequency & \\
$\omega_{n}$ & Theoretical natural frequency & \\
$\omega_{n}^{e x p}$ & Measured natural frequency & $n\left(n^{2}-1\right)$ \\
$\Omega_{n}$ & Dimensionless natural frequency & \\
$\Omega$ & Dimensionless angular frequency & $\omega_{n}^{\text {exp }} / \omega_{n}$ \\
$\chi$ & Ratio of frequencies &
\end{tabular}

\section{Major abbreviations}

\begin{tabular}{ll} 
Symbol & Meaning \\
\hline \hline DI & Deionised \\
EOF & Electroosmotic flow \\
EW & Electrowetting \\
EDL & Electric double layer \\
ITO & Indium tin oxide \\
LPCVD & Low pressure chemical vapour deposition \\
MALDI-TOF & Matrix-assisted laser desorption/ionization-time of flight \\
ONO & Silicon (oxide-nitride-oxide) \\
PDMS & Polydimethylsiloxane \\
PECVD & Plasma enhanced chemical vapour deposition \\
TCL & Triple contact line
\end{tabular}





\section{Summary}

Microfluidics or lab on chip technology, has developed itself significantly during the past 25 years. Now it has become a robust tool to manipulate tiny amounts of fluid or gas for various applications. Microfluidics is the process of transporting, mixing, separating small amounts of fluid in microchannels. Also chemical reactions can be carried out in a well controlled way. With this technology major advancements in point-of-care diagnostics, biotechnology, pharmaceuticals, public health and agriculture has been achieved. The basic ingredients of microfluidics are microchannels, micropumps to transport small amounts of fluid or droplets, electronics to control the flow and various sensors and detectors for chemical analysis. The research described in this thesis deals with flow in microchannels and in small drops. In both cases the flow is controlled by electric fields.

Chapter 3 and 4 describe the research on microchannels. In these channels we generate and control an electro osmotic flow using gate electrodes embedded in the channel wall. By varying the zeta potential i.e. the potential at the channel wall we are able to control the flow speed near that wall. By applying an opposite voltage to opposing walls a shear flow is created inside the channel. The strength of the shear rate will depend upon the zeta-potential near both walls. However, this zeta-potential is difficult to measure. To determine this potential, we first study homogeneous electroosmotic flow through a microchannel, made of glass or glass-PDMS, by displacing an electrolyte solution at given concentration with the same electrolyte at a different concentration via an external elec- 
tric field along the channel axis. When the voltage drop over the channel is kept constant, the electric current through the channel varies during the displacement process. We propose a simple analytical model that describes the time dependence of the current. With this model, which is also applicable at relatively high wall potentials, we are able to quantify the electroosmotic flow velocity and to determine the surface charge on the microchannel walls from the measured current behaviour, as well as the zeta potential at given local electrolyte concentration.

The zeta-potential can be modified not only using gate electrodes but also by chemical modification of the channel walls. Chemical modification is obtained by treating one of the channel walls with a cationic polymer. This causes a change of the equilibrium ion concentrations near the wall which results in a considerable change of the surface charge and the zeta-potential at that wall. By varying the surface potential, via chemical modification or by using gate electrodes, a controllable shear stress is applied to the liquid in the channel. The strength of this shear stress depends on the applied field which drives the electroosmotic shear flow and in case of using gate electrodes, on the gate voltage. Although the range of applicable shear stresses is still limited, such a device can be used to study the shear behaviour of complex fluids or soft matter in situ in lab on chip devices.

The study of the dynamic behaviour of sessile and sandwiched drops is of great importance for the understanding of the processes that occur in microfluidics. In chapter 5, 6 and 7 we study flow patterns inside sessile drops on a thin dielectric layer, when the liquid-air interface of the drop is oscillated by electrowetting. The frequency of this oscillation is determined by the frequency of the applied AC voltage. We show that the strength of the flow field in polar direction correlates with the eigen modes of the drop interface oscillation. The azimuthal symmetry of this flow can be broken by introducing pinning sites along the contact line of the drop. For each pinning site, two vortices occur, independent of the applied frequency.

These flow fields inside the drop facilitate not only mixing of the liq- 
uid but also pre-concentration of dispersed particles. When the frequency of the applied voltage is gradually increased to typically $10 \mathrm{kHz}$ the azimuthal flow vortices move towards the contact line near the pinning site. These vortices sweep micro/nano particles, or even DNA dispersed in the droplet, towards the pinning sites. As a result, the particle concentration increases locally inside the drop by a factor of about ten.

One of the applications of internal drop flow is the suppression of the so called coffee stain effect. The coffee stain effect is the formation of circular unevenly distributed residue patterns when a coffee drop evaporates on a solid surface. This is caused by the motion of the suspended particles towards the contact line during evaporation. At the rim these particles settle and prevent the contact line to shrink any further. In chapter 6 we show that electrowetting can suppress stains of colloidal particles and DNA solute when the frequency of the applied AC voltage is chosen in the range from a few $\mathrm{Hz}$ to a few tens of $\mathrm{kHz}$. We also identify the potentials and limitations of this method, by investigating the influence of factors as solute particle size and concentration, and amplitude of the contact line motion. Moreover, in order to emphasize the importance of suppressing these stains in (bio-) chemical analysis, we present a MALDI-TOF experiment with a homogeneous (after stain suppression) and a heterogeneous residue (without stain suppression) of poly ethylene glycol. The homogeneous sharp spot of residue resulted in an increase in the signal strength of two orders of magnitude.

In droplet based microfluidics, drops are often sandwiched between two parallel positioned hydrophobic glass plates where they can be moved, merged together or split, using electrowetting. In chapter 7, we study the oscillatory behaviour of such a sandwiched drop. The oscillations are driven by periodic modulation of the contact line motion again using AC electrowetting. The drop oscillates in different shape modes depending upon the frequency of the applied voltage. The oscillations have half the excitation frequency of the contact line, i.e. they oscillate with the frequency of the applied voltage. This is a characteristic behaviour for parametrically excited oscillations. The drop response for sinusoidal driving, is 'stop and go' with a substantial deviation from harmonic behaviour, es- 
pecially for the oscillation modes at low frequency. Although the shape modes follow our simple theoretical model, the resonances appear at substantially lower frequencies than expected. We attribute this to pinning effects of the contact line on the substrate. 


\section{Samenvatting}

Microfluidics, ook wel 'lab-on-a-chip' technologie genoemd, heeft zich de afgelopen 25 jaar sterk ontwikkeld. Het is een robuuste techniek waarbij kleine hoeveelheden vloeistof of gas door kanaaltjes of in containertjes van micrometer afmetingen gemanipuleerd worden. In microfluidics worden kleine hoeveelheden vloeistof getransporteerd, gescheiden of juist gemengd. Ook chemische reacties kunnen in een 'lab-on-a-chip' goed gecontroleerd uitgevoerd worden. Dankzij deze technologie is er de laatste jaren veel vooruitgang geboekt op het gebied van point-of-care medische diagnostiek, biotechnologie, farmaceutische producten, volksgezondheid en landbouw onderzoek. De basisbenodigdheden voor microfluidics zijn microkanalen, micropompjes om de vloeistof of druppels te transporteren, elektronica voor het controleren van de stroming en verschillende soorten sensoren en detectoren, dit alles zo veel mogelijk geintegreerd op een enkele chip. In dit proefschrift wordt onderzoek beschreven naar het stromingsgedrag van (complexe) vloeistoffen zowel in microkanalen alsook in kleine druppels en hoe dit via electrische velden beinvloed en gestuurd kan worden.

De hoofdstukken 3 en 4 beschrijven het onderzoek aan microkanalen. Hierbij genereren wij een elektro-osmotische stroming en proberen deze te manipuleren met behulp van zogenaamde gate elektrodes, die in de wanden van het kanaal aangebracht zijn. Via deze elektrodes kunnen we de electrische potentiaal in de vloeistof aan de wand (de zeta-potentiaal) regelen en daarmee de locale stroomsnelheid van de vloeistof. Door tegenover elkaar liggende wanden op een tegengestelde potentiaal of span- 
ning te brengen, kunnen wij een afschuifstroming in het kanaal creëren. De sterkte van deze afschuifstroming wordt bepaald door het verschil in zeta-potentiaal aan de twee wanden. Deze is echter niet makkelijk te meten. Om deze te kunnen bepalen, bestuderen wij eerst een homogene elektro-osmotische stroming in een microkanaal van glas of PDMS. Door een elektrisch veld in de lengte richting van het kanaal aan te brengen kan een elektrolytoplossing met een specifieke concentratie verdrongen worden door eenzelfde oplossing maar met een andere concentratie. Als het elektrisch veld constant gehouden wordt, zal de elektrische stroom in het kanaal tijdens het verdringingsproces varieren. Wij presenteren een simpel analytisch model, dat een verband legt tussen deze varierende stroom en de zeta-potentiaal. Met dit model, dat ook toepasbaar is bij relatief hoge potentiaal in de vloeistof, kunnen wij de zeta potentiaal en daarmee de elektro-osmotische stroomsnelheid kwantificeren. Daarnaast kunnen wij uit de gemeten stroom-tijd karakteristiek ook de oppervlaktelading aan de wand van het kanaal vaststellen.

De zeta-potentiaal aan de kanaalwanden (die we willen varieren om de sterkte van de afschuifstroming te regelen) kan niet alleen aangepast worden door gebruik te maken van gate elektrodes maar ook door middel van chemische modificatie van de kanaalwanden. Hierbij wordt één van de kanaalwanden behandeld met een kationische polymeer, waardoor het ionen evenwicht aan de wand dusdanig verandert dat de oppervlaktelading en de zeta-potentiaal ook sterk veranderen.

Door de zeta-potentiaal te variëren, hetzij via chemische modificatie of met behulp van gate elektrodes, kan de vloeistof in het kanaal een controleerbare afschuifspanning opgelegd worden. De grootte van deze spanning is afhankelijk van de sterkte van het electrisch veld langs de kanaal-as én -in geval van gate electrodes- van de spanning op deze elektrodes. Hoewel het bereik van afschuifspanningen nog beperkt is, kunnen met behulp van dit kanaal complexe vloeistoffen of zachte materie in situ in lab-on-a-chip apparatuur onder afschuiving onderzocht worden.

Het bestuderen van het dynamisch gedrag van een druppel op een substraat is van groot belang voor het begrip van de processen welke zich in microfluidics afspelen. In hoofdstuk 5, 6 en 7 onderzoeken we het 
stromingspatroon in druppels op een dun diëlectricum. Het oppervlak van deze druppels wordt hierbij in trilling gebracht door electrowetting (EW). De frequentie van deze trilling wordt bepaald door de frequentie van de elektrische aandrijfspanning. Wij demonstreren dat de sterkte van het stromingsveld in de polaire richting correleert met de eigen modes van druppeloppervlakte-oscillaties. De azimutale symmetrie van het stromingsveld kan verbroken worden door de contactlijn van de druppel op bepaalde plaatsen vast te hechten of te pinnen aan het substraat. Zo'n plaats noemen we een pinpunt (Engels: pinning site). Ieder pinpunt veroorzaakt twee wervels, ongeacht de frequentie van de opgelegde spanning.

Met deze stroming kunnen we de vloeistof in de druppel mengen. En als er in de vloeistof kleine deeltjes gedispergeerd zijn, kunnen deze door middel van ditzelfde stromingspatroon op een speciefieke plaats samen gebracht worden, hetgeen wel pre-concentratie genoemd wordt. Wanneer de frequentie van de aangelegde wisselspanning geleidelijk wordt verhoogd, van zo'n $10 \mathrm{~Hz}$ tot een aantal $\mathrm{kHz}$ dan verplaatst de wervel zich in de richting van het pinpunt op de contactlijn. Deze wervel trekt de aanwezige micro/nano deeltjes, zelfs DNA, met zich mee naar dat pinpunt. Als gevolg hiervan kan de deeltjesconcentratie lokaal wel vertienvoudigen.

Eén van de toepassingen van deze interne druppelstroming is het onderdrukken van het 'koffiekringeffect'. Dit effect verwijst naar het onregelmatige residupatroon dat op de ondergrond achterblijft als een koffie druppel verdampt is. Dit komt doordat de deeltjes tijdens het verdampen naar de rand van de druppel gedreven worden en daar vervolgens het verder slinken van de contactlijn verhinderen. In hoofdstuk 6 laten we zien dat dit effect met electrowetting verhinderd kan worden, wanneer de frequentie van de wisselspanning in het juiste bereik gekozen wordt: ongeveer $1 \mathrm{~Hz}$ tot een tiental $\mathrm{kHz}$. Bovendien onderzoeken we de mogelijkheden en beperkingen van deze methode, door de invloed van factoren als deeltjesgrootte en concentratie en de amplitude van de contactlijnbeweging te bekijken. Ook wordt een MALDI-TOF experiment beschreven met een heterogeen residu polyethyleenglycol ten gevolge van 
het koffiekring effect en een homegeen residu waarbij tijdens het prepareren het kring effect onderdrukt werd. Hiermee wordt het belang van het onderdrukken van het koffiekringeffect voor (bio-) chemische analysedoeleinden aangetoond. De homogene scherpe residustip leverde een 100 keer grotere signaalsterkte op.

In op druppel transport gerichte microfluidics worden de druppels gewoonlijk gevangen gehouden tussen twee parallel geplaatste hydrofobe glasplaatjes, waarbij ze met behulp van electrowetting worden verplaatst, samen kunnen vloeien of juist opgesplitst kunnen worden. Ook van deze druppels hebben wij, in hoofdstuk 7 , het oscillatie-gedrag onderzocht. Hierbij wordt de contactlijnbeweging van de druppel periodiek gemoduleerd met behulp van wisselstroom electrowetting. Afhankelijk van de toegepaste frequentie oscilleert de druppel in verschillende basispatronen. Hierbij is de frequentie van de oscillaties precies de helft van de excitatiefrequentie van de contactlijn, met andere woorden de druppel oscilleert met de frequentie van de aangelegde spanning. Dit is karakteristiek voor parametrisch geëxciteerde oscillaties. De druppelrespons op een sinusoïdale aandrijving is intermitterend, waarbij er een substantiële afwijking van het sinusoïdaal gedrag optreedt, vooral bij lagere oscillatie frequenties. Hoewel de gevonden patronen overeenkomen met ons simpel theoretisch model, treden de resonanties bij aanzienlijk lagere frequenties op, dan door het model voorspeld wordt. Wij vermoeden dat dit verschil veroorzaakt wordt door de pineffecten van de contactlijn op het substraat. 


\section{Bibliography}

[1] Hendrik Bruus, Theoretical microfluidics, Oxford university press, Oxford (2007)

[2] Hunter R. J., Foundations of colloid science, 2, Clarendon Press, Oxford (1989)

[3] Brian J. Kirby, Ernst F. Hasselbrink Jr., Electrophoresis 25, 187, (2004)

[4] M. J. Spaarnaay, J. Electroanal. Chem., 37, (1972)

[5] Dezsö Boda, Kwong-Yu Chan, and Douglas Henderson, J. Chem. Phys. 109, 7362 (1998)

[6] Jan C. T. Eijkel and Albert van den Berg, Chem. Soc. Rev. 39, 957973, (2010)

[7] Jacob Israelachvili, Intermolecular and surface forces, Academic Press, Third Edition (2011)

[8] Reto B. Schoch, Jongyoon Han, Philippe Renaud, Rev. mod. Phys. 80, (2008)

[9] Overbeek, J. T. G., Colloid Science 1, Elsevier, Amsterdam (1952)

[10] H. Fricke, Phil. Mag. 14, pp. 310-318, (1932)

[11] F. F. Reuss. Mem. Soc. Impr. Natural. Moskou 2, 327, (1809) 
[12] D. Burgreen, and F. R. Nakache, J. Phys. Chem. 68, 1084-1091, (1964)

[13] Smoluchowski, M. V., Z. Phys. Chem., Stoechiom. Verwandtschaftsl. 92, 129, (1916)

[14] Ren, L., Canseco, C. E., Li, D., J. Coll. Int. Sci. 250, 238-242, (2002)

[15] Dileep Mampallil, Dirk van den Ende, Frieder Mugele, Electrophoresis 31, 563-569 (2010)

[16] Pierre-Gilles de Gennes, Francoise Brochard-Wyart, David Quere, Capillarity and Wetting Phenomena: Drops, Bubbles, Pearls, Waves (2004)

[17] P. G. de Gennes, Rev. Mod. Phys. 57, (1985)

[18] Young T., Philos. Trans. R. Soc. London, 95, (1805)

[19] Mugele, F., Baret, J. C., J. Phys. Condens. Matter 17, R705R774,(2005)

[20] Buehrle J., Herminghaus S. and Mugele F., Phys. Rev. Lett. 91 086101, (2003)

[21] Anthony Quinn, Rossen Sedev, and John Ralston, J. Phys. Chem. B 107, 1163-1169, (2003)

[22] Charles W. Monroe, Michael Urbakh, and Alexei A. Kornyshev, J. Electrochem. Soc., 156, 21-28, (2009)

[23] C. K. Hua and I. S. Kang, K. H. Kang, H. A. Stone, Phys. Rev. E 81, 036314, (2010)

[24] Chun Yang, Dongqing Li, A: Physicochem. Eng. Aspects 143, 339353 (1998)

[25] Armand Ajdari, Phys. Rev. E, 61, (2000) 
[26] W.E. Morf, O.T. Guenat, N.F. de Rooij, Sens. Actuators B 72, 266$272,(2001)$

[27] V. Pretorius, B. J. Hopkins, and J. D. Schieke, J. Chromatogr. 99, 23, (1974)

[28] Kyohei Terao, Hiroyuki Kabata and MasaoWashizu, J. Phys.: Condens. Matter 18, S653-S663, (2006)

[29] John M. Edwards, Mark N. Hamblin, Hernan V. Fuentes, Bridget A. Peeni, Milton L. Lee, and Adam T. Woolley, Aaron R. Hawkins, Biomicrofluidics 1, 014101, (2007)

[30] Jia-Kun Chen, Ruey-Jen Yang, Microfluid Nanofluid, DOI 10.1007/s10404-008-0307-3, (2008)

[31] Ramos, A., Morgan, H., Green, N. G. and Castellanos, A., J. Phys. D: Appl. Phys. 31, 2338-2353, (1998)

[32] Squires T. M., Bazant M. Z., J. Fluid Mech. 509, 217-252, (2004)

[33] Ajdari, A., Phys. Rev. Lett. 75, 755-758, (1995)

[34] Ajdari, A., Phys. Rev. E 65, 016301, (2002)

[35] Moorthy, J., C. Khoury, J. S. Moore, and D. J. Beebe, Sens. Actuators B 75, 223, (2001)

[36] Hiroyuki Katayama, Yasushi Ishihama, and Naoki Asakawa, Anal. Chem. 70, 5272-5277, (1998)

[37] Yan Liu, Joseph C. Fanguy, Justin M. Bledsoe, and Charles S. Henry, Anal. Chem. 72, 5939-5944, (2000)

[38] Richard B. M. Schasfoort, Stefan Schlautmann, Jan Hendrikse, Albert van den Berg, Science 286, 942, (1999)

[39] Minglin M, Randal M. Hill, Current Opinion in Colloid and Interface Science 11, 193-202, (2006) 
[40] Supreet S. Bahga, Olga I. Vinogradova and Martin Z. Bazant, J. Fluid Mech. 644, 245-255, (2010)

[41] Laurent Joly, Christophe Ybert, Emmanuel Trizac, and Lyderic Bocquet, Phys. Rev. Lett. 93, 257805, (2004)

[42] Shchekin, A.K. and Borisov, V.V., Colloid J. 67, 774-787, (2005)

[43] Zhijun Jiang and Derek Stein, Langmuir 26, 8161-8173, (2010)

[44] Rogier B. H. Veenhuis, Egbert J. van der Wouden, Jan W. van Nieuwkasteele, Albert van den Berg and Jan C. T. Eijkel, Lab Chip 9, 3472-3480, (2009)

[45] Chia-Yen Lee, Gwo-Bin Lee, Lung-Ming Fu, Kuo-Hoong Lee, J. Micromech. Microeng. 14, 1390-1398, (2004)

[46] Hsin-Yu Wu, Cheng-Hsien Liu, Sens. Actuators A 118, 107-115, (2005)

[47] E.J. van der Wouden, D.C. Hermes, J.G.E. Gardeniers, and A. van den Berg, Lab. Chip 6, 1300, (2006)

[48] Rohit Karnik, Rong Fan, Min Yue, Deyu Li, Peidong Yang, and Arun Majumdar, Nano Lett. 5, (2005)

[49] Rong Fan, Min Yue, Rohit Karnik, Arun Majumdar, and Peidong Yang, Phys. Rev. Lett. 95, 086607, (2005)

[50] Abraham D. Stroock, Marcus Weck, Daniel T. Chiu, Wilhelm T. S. Huck, Paul J. A. Kenis, Rustem F. Ismagilov, and George M. Whitesides, Phys. Rev. Lett. 84, (2000)

[51] Mingyan He, J. Scott Edgar, Gavin D. M. Jeffries, Robert M. Lorenz, J. Patrick Shelby, and Daniel T. Chiu, Anal. Chem. 77, 1539-1544, (2005)

[52] Helen Song, Delai L. Chen, and Rustem F. Ismagilov, Angew Chem Int Ed Engl. 45, 7336-7356, (2006) 
[53] H. Gu , F. Malloggi, S. A. Vanapalli, F. Mugele, Appl. Phys. lett. 93, (2008)

[54] S. Utada, E. Lorenceau, D. R. Link, P. D. Kaplan,H. A. Stone, A. Weitz. Science 308, 537, (2005)

[55] Jung Min Oh, Sung Hee Ko, and Kwan Hyoung Kang, Langmuir 24, 8379-8386, (2008)

[56] Mei-Feng Lai, Chiun-Peng Lee, Chun-Neng Liao, and Zung-Hang Wei, Appl. Phys. Lett. 94, 154102, (2009)

[57] Prosenjit Sen and Chang-Jin "CJ" Kim, Langmuir 25, 4302-4305, (2009)

[58] F. Mugele and J.C. Baret, D. Steinhauser, Appl. Phys. Lett. 88, 204106, (2006)

[59] Sung Hee Ko, Horim Lee, and Kwan Hyoung Kang, Langmuir 24, 1094, (2008)

[60] R. Malk, Y. Fouillet , L. Davoust, Sens. Actuators B, (2010) doi:10.1016/j.snb.2009.12.066

[61] F. Mugele, A. Staicu, R. Bakker, and D. van den Ende, Lab Chip 11, 2011-2016, (2011)

[62] Pablo Garcia-Sanchez, Antonio Ramos, and Frieder Mugele, Phys. Rev. E 81, 015303R, (2010)

[63] Horim Lee, Sungchan Yun, Sung Hee Ko, and Kwan Hyoung Kang, Biomicrofluidics 3, 044113, (2009)

[64] H.B. Eral, D. Mampallil, M.H.G. Duits and F. Mugele, Soft Matter 7, 4954-4958, (2011)

[65] F. Li and F. Mugele, Appl. Phys. Lett. 92, (2008) 
[66] D. J. C. M. 't Mannetje, C. U. Murade, D. van den Ende, and F. Mugele, Appl. Phys. Lett. 98, 014102, (2011)

[67] Joost Melai, Cora Salm, Sander Smits, Jan Visschers and Jurriaan Schmitz, J. Micromech. Microeng. 19, 065012, (2009)

[68] https://www51.honeywell.com

[69] L. Kerkache, A Layadi, E Dogheche and D Remiens, J. Phys. D: Appl. Phys. 39 184-189, (2006)

[70] A. Boogaard, A.Y. Kovalgin, R.A.M. Wolters, Microelectronic Eng. 86, 1707-1710, (2009)

[71] Say Hwa Tan, Nam-Trung Nguyen, Yong Chin Chua, and Tae Goo Kang, Biomicrofluidics 4, 032204, (2010)

[72] Honest Makamba, Jin Ho Kim, Kwanseop Lim, Nokyoung Park, Jong Hoon Hahn, Electrophoresis 24, 3607-3619, (2003)

[73] http://rsbweb.nih.gov/ij/

[74] F.Sbalzarini and P. Koumoutsakos, J. Struct. Biology 151, 182-195, (2005)

[75] Squires, T. M., Quake, S. R., Rev. Mod. Phys., 77, 977-1026, (2005)

[76] Burgreen, D., Nakache, F., J. Phys. Chem., 68, 1084-1091, (1964)

[77] Bruin, G. J. M., Electrophoresis, 21, 3931-3951, (2000)

[78] Russel, W. B., Saville, D. A., Schowalter, W. R., "Colloidal Dispersions", Cambridge University Press, (1989)

[79] Schoch, R. B., Han, J., Renaud, P., Rev. Modern Phys., 80, 839-883, (2008)

[80] Lee, G. B., Lin, C. H., Lee, K. H., Lin, Y. F., Electrophoresis, 26, 4616-4624, (2005) 
[81] Sinton, D., Canseco, C. E., Ren, L., Li, D., J. Coll. Int. Sci., 254, 184-189, (2002)

[82] Huang, X., Gorden, M. J., Zare, R. N., Anal. Chem., 60, 1837-1838, (1988)

[83] Ren, L., Canseco, C. E., Li, D., J. Coll. Int. Sci., 250, 238-242, (2002)

[84] Arulanandam, S., Li, D., J. Coll. Int. Sci., 225, 421-428, (2000)

[85] Ren, L., Canseco, C. E., Li, D., J. Coll. Int. Sci., 242, 264-271, (2001)

[86] Ren, L., Masliyah, J., Li, D., J. Coll. Int. Sci., 257, 85-92, (2003)

[87] Wang, C., Wong, T. N., Yang, C., Ooi, K. T., Int. J. Heat and Mass Trans., 50, 3115-3121, (2007)

[88] Devasenathipathy, S., Bharadwaj, R., Santiago, J.G., Exp. Fluids, 43, 959-967, (2007)

[89] Barz, D. P. J., Vogel, M. J., Steen, P. H., Langmuir, 25, 1842-1850, (2009)

[90] B. Ladoux and P. S. Doyle, Europhys. Lett. 52, pp. 511-517 (2000)

[91] A Ansari, CM Jones, ER Henry, J Hofrichter, and WA Eaton, Science 256, 1796-1798, (1992)

[92] Nitzan Resnick and Michael A. Gimbrone, Jr., The FASEB Journal 9, 874, (1995)

[93] Justice M. Thwala, Jim W. Goodwin, and Paul D. Mills, Langmuir 25, 12926-12936, (2009)

[94] L. E. Silbert and J. R. Melrose, J. Rheol. 43, (1999) 
[95] Itai Cohen, Thomas G. Mason, and David A. Weitz, Phys. Rev. Lett. 93, (2004)

[96] Michael Kogan, and Michael J. Solomon, Langmuir, 26 1207-1213, (2010)

[97] P. A. Smith, G. Petekidis, S. U. Egelhaaf, and W. C. K. Poon, Phys. Rev. E, 76, 041402, (2007)

[98] Hiroyuki Katayama, Yasushi Ishihama, and Naoki Asakawa Anal. Chem., 70, 5272-5277, (1998)

[99] Yan Liu, Joseph C. Fanguy, Justin M. Bledsoe, and Charles S. Henry, Anal. Chem., 72, 5939-5944, (2000)

[100] Winky L WHau, Dieter W Trau, Nikolaus J Sucher, Man Wong and Yitshak Zohar, J. Micromech. Microeng., 13, 272-278, (2003)

[101] Susan L. R. Barker, David Ross, Michael J. Tarlov, Michael Gaitan, and Laurie E. Locascio, Anal. Chem., 72, 5925-5929, (2000)

[102] Rob Haselberg Gerhardus J. de Jong Govert W. Somsen, J. Sep. Sci., 32, 2408-2415, (2009)

[103] Abraham D. Stroock, Marcus Weck, Daniel T. Chiu, Wilhelm T. S. Huck, Paul J. A. Kenis, Rustem F. Ismagilov, and George M. Whitesides, Phys. rev. Lett. 84, 3314-3317, (2000)

[104] M. Crosnier de Bellaistre, O. Mathieu, J. Randon, J.L.Rocca , Journal of Chromatography A, 971, 199-205, (2002)

[105] Zhijun Jiang and Derek Stein, Langmuir, 26, 8161-8173, (2010)

[106] Hsin-Yu Wu, Cheng-Hsien Liu,Sensors and Actuators A, 118, 107$115,(2005)$

[107] Dileep Mampallil, Dirk van den Ende, Frieder Mugele, Electrophoresis, 31, 563-569, (2010) 
[108] K.P. Nichols and H.J.G.E. Gardeniers, Proc of MicroTAS, 582-584 (2006)

[109] L. Rayleigh, Proc. R. Soc. (London) 29, 71 (1879)

[110] M. Strani and F. Sabetta, J. Fluid Mech. 141, 233-247, (1984)

[111] Irina S. Fayzrakhmanova and Arthur V. Straube, Phys. Fluids 21, 072104, (2009)

[112] Chun-Che Lin, Jue-Liang Hsu, Gwo-Bin Lee, Microfluid Nanofluid 10:481-511, (2011)

[113] Mingyan He, Chenhang Sun, and Daniel T. Chiu, Anal. Chem., 76, 1222-1227, (2004)

[114] Sung Kwon Cho, Yuejun Zhao and Chang-Jin "CJ" Kim, Lab Chip, 7, 490-498, (2007)

[115] Hye-Na Kim, Ji-Hwan Kang, Woo-Min Jin and Jun Hyuk Moon, Soft Matter, 7, 2989, (2011)

[116] X. Noblin, A. Buguin and F. Brochard-Wyart, Phys. Rev. Lett., 94, $166102(2005)$

[117] Kyle D. Squires and John K. Eaton, Phys. Fluids A 3, 1169 (1991)

[118] Jeong-Yeol Yoon and Robin L. Garrell, Anal. Chem., 75, 50975102, (2003)

[119] Robert D. Deegan, Olgica Bakajin, Todd F. Dupont, Greg Huber, Sidney R. Nagel, Thomas A. Witten, Nature 389, 827-829, (1997)

[120] R. Blossey and A. Bosio, Langmuir 18, 2952 (2002)

[121] V. Dugas, J. Broutin, and E. Souteyrand, Langmuir, 21, 9130, (2005) 
[122] S. Weidner, P. Knappe and U. Panne, Anal. Bioanal. Chem. 401, (2011)

[123] E. Tekin, B. de Gans, and U. Schubert, J. of Materials Chem. 1, 2627 (2004)

[124] W. D. Ristenpart, P. G. Kim, C. Domingues, J. Wan, and H. A. Stone, Phys. Rev. Lett. 99 (2007)

[125] S. J. Kim, K. H. Kang, J.-G. Lee, I. S. Kang, and B. J. Yoon, Anal. Chem. 78, 5192, (2006)

[126] Jung-Hoon Kim, Sung II Ahn, Jae Hyun Kim and Wang-Cheol Zin, Langmuir 23, 6163-6169, (2007)

[127] A. M. Cazabat and G. Guena, Soft matter 6, 2591-2612, (2010)

[128] R. D. Deegan, Phys. Rev. E. 61, (2000)

[129] E. Rio, A. Daerr, F. Lequeux and L. Limat, Langmuir 22, 3186, (2006)

[130] H. Bodiguel, Frederic Doumenc and Beatrice Guerrier, Langmuir 26, 10758-10763, (2010)

[131] F. Li and F. Mugele, Appl. Phys. Lett. 92, 244108 (2008)

[132] N. D. Denkov O. D. Velev P. A. Kralchevsky I. B. Ivanov, H. Yoshimura, and K. Nagayama, Langmuir 8, 3183-3190, (1992)

[133] A. D. Nikolov and D. T. Wasan, Ind. Eng. Chem. res. 48, 23202326, (2009)

[134] J. Kleinert, S. Kim and O. D. Velev, Langmuir 26, 10380-10385, (2010)

[135] Andrea Prosperetti, J . Fluid Mech. 100, pp. 333-347, (1980)

[136] M. Strani and F. Sabetta, J. Fluid Mech. 141,233-247, (1984) 
[137] A. A. Amsden, G. F. Bertsch, F. H. Harlow, and J. R. Nix, Phys. Rev. Lett. 35, 905-908 (1975)

[138] R. H. Durisen et al., Astrophys. J. 305, 281 (1986)

[139] Bojan Vukasinovic, Marc K. Smith and Ari Glezer, J. Fluid. Mech. 587, 395-423, (2007)

[140] Norman J. Holter, Wilford R. Glasscock, J. of acoustical society of America, 24, (1952)

[141] Ken Adachi and Ryuji Takaki, J. of physical society of Japan, 53, pp 4184-4191, (1984)

[142] Ryuji Takaki and Ken Adachi, J. of physical society of Japan, 54, pp 2462-2469, (1985)

[143] D. L. Cummings and D. A. Blackburn, J . Fluid Mech., 224, pp. 395416, (1991)

[144] Yoshiyasu N and Takaki R, J. of physical society of Japan, 65, (1996)

[145] Morihiro Okada and Minoru Okada, Exp Fluids 41, 789-802, (2006)

[146] C. L. Shen, W. J. Xie, and B. Wei, Phys. Rev. E 81, 046305 (2010)

[147] P. Brunet and j. H. Snoeijer, Eur. Phys. j. Special Topics 192, $207-$ 226, (2011)

[148] X. Noblin, A. Buguin and F. Brochard-Wyart, Eur. Phys. J.E., 14, 395-404 (2004)

[149] A. A. Alabuzhev and D. V. Lyubimov, J. Appl. Mech. Tech. Phys. 48, 686 (2007)

[150] Ramon Pericet-Camara, Georg Papastavrou, Sven H. Behrens, and Michal Borkovec, J. of Phys. Chem. B 108, 19467-19475, (2004) 



\section{Acknowledgements}

During the past four years, I have got valuable assistances from many people in the development and execution of this thesis project. I would like to use this space for expressing my gratitude to them.

Foremost, I would like to thank Dirk and Frieder. I must say that I have learnt lots of things from Dirk. He helped me throughout my PhD. In fact, most of the theoretical work in my thesis was done by Dirk. Without his help, I would not be able to write-up my thesis successfully. I owe my deepest gratitude to Frieder also for guiding me in the $\mathrm{PhD}$ research. I appreciate the suggestions and critical comments he gave me. It kept me motivated and helped me to improve the results. I express my gratitude to the committee members for reading and evaluating my thesis.

I would like to extend my gratitude to Cock Harteveld, Daniel, Klaas, Mariska, Michel and people in the glass workshop. Michel was very supporting posing useful comments during group meetings. Daniel was very quick in making samples from the cleanroom. It actually helped me a lot. I thank Annelies for arranging the administrative matters during my stay in the group. Many thanks to Jean-Philippe Frimat and Séverine Le Gac for providing cells for my experiments. I am very thankful to Prof. Jan Eijkel, Egbert van der Wouden and Prof. Han Gardeniers for fruitful discussions, Nikolay Dimov for performing MALDI experiments and Joost Melai, Victor Blanco Carballo, Bijoy for helping to coat thin SU8 insulation layers.

I am very thankful to the authors of the free scientific software like ImageJ, ETH-MOSAIC particle tracker, MikeTex and Texmaker. 
I would like to express my gratitude to Johan Meersschaut who was my supervisor during my research work in K. U. Leuven. I was impelled by his inspiration.

The support from my friends and colleagues, namely from Adrian, Agata, Alberto, Arun Banpurker, Arun, Burak, Chandra, Daniel Ebeling, Dhirendra, Dieter, Eko, Florent, Gor, Hao, Helmut, Jane, Jolet, Jung, Omkar, Pablo, Rielle, Sissi, Siva and Williem was very valuable. I enjoyed many fruitful experiments carried out with Burak.

There are many people from outside the group who were very supporting. The time I spent with Rajesh and Jithin was very great. Personally, it helped me to enjoy the time here during the $\mathrm{PhD}$-tension. At this point, I also remember about the moral supports from Ajay, Benson, Bijilash, Febin, Hrudya, Maneesh, Naveen, Nijil, Praveen, Rejish, Sajan, Sumesh, Vidhu, Yasar etc.

Without thanking Devadas sir, Sandhya teacher and Harikrishnan sir, this acknowledgement section would not be complete. In fact their boost and inspiration helped me to to step into IIT Madras and to gain further academic achievements.

I am extremely thankful to mama, dad and Julian who were very supporting and helpful. I remember the homely feeling and of course the Polish food I enjoyed. I am very thankful to Emilia, the love and luck in my life. I believe that the results in the lab came after meeting her. I owe my deepest gratitude to chachan and amma. I remember the times, they were working hard from dawn to dusk to bring us up. They were very motivating and supporting even during the difficult times. Without their love and hard work I would not be able to write this thesis. I am happy to have the motivation from Deepa, Sajith chettai, Leena and Sibi chettai.

Finally, once again I thank them all. Thank you!

\section{Dileep Mampallil}

17-Aug-2011 


\section{Publications}

- Dileep Mampallil, Dirk van den Ende and Frieder Mugele, A simple method to determine surface charge in microfluidic channels, Electrophoresis 31, 563569, (2010).

- H.B. Eral*, Dileep Mampallil ${ }^{*}$, M.H.G Duits and F. Mugele, Suppressing the coffee stain effect: how to control colloidal self-assembly in evaporating drops using electrowetting, Soft Matter 7, 49544958, (2011)* Equally contributed

- Dileep Mampallil, Dirk van den Ende and Frieder Mugele, Controlling flow patterns in oscillating sessile drops by breaking azimuthal symmetry (Under review by Appl. Phys. Lett.)

- Dileep Mampallil, Dirk van den Ende and Frieder Mugele, Electro osmotic shear flow in micro channels (In preparation)

- Dileep Mampallil, Dirk van den Ende, H. B. Eral, A. Staicu and Frieder Mugele, Oscillations of a sandwiched drop by AC electrowetting (In preparation)

- Dileep Mampallil, H. B. Eral, Dirk van den Ende and Frieder Mugele, Suppressing the coffee stain effect using electrowetting: influence of solute concentration and size (In preparation)

- Dileep Mampallil, Dirk van den Ende and Frieder Mugele, Sample

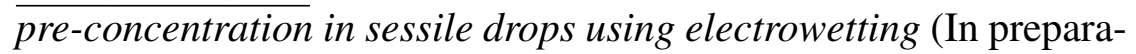
tion) 
- H. B. Eral, D. Mampallil, N. Dimov, H. Gardeniers, F. Mugele Overcoming the coffee stain problem in MALDI-MS analyses by electrowetting (In preparation)

\section{Conference Paper(s)}

- D. Mampallil, H.T.M. van den Ende, F. Mugele, Electro osmotic shear flow in microchannels, Proc. of the 14th International Conference on Micro Total Analysis Systems (MicroTAS), Groningen, 1124,2010

\section{$\operatorname{Patent}(\mathbf{s})$}

- H.B. Eral, Dileep Mampallil and Frieder Mugele, Coffee stain effect suppression, International patent application, PCT/NL2011/050328 (Patent pending) 


\section{About the author}

Dileep Mampallil was born on $20^{t h}$ May 1981 in Kanhirapuzha, India. He grew up in Padiyoor, a village in north Kerala, India. He received his BSc degree in physics from Kannur University, India. Besides studying, in Mahathma Gandhi College, Iritty, he was an active member in National Service Scheme and also functioned as the college magazine editor. In 2004, he obtained MSc. degree in physics from Indian Institute of Technology (IIT) Madras, India. Afterwards, he did a one year research at $\mathrm{Nu}-$ clear Solid-state Physics group in Katholieke Universiteit Leuven on field cooling effect in magnetic multilayers. In 2006-07 he worked as a lecturer in Mahathma Gandhi College, Iritty where he taught electrodynamics in BSc level. In 2010 he got married. He completed four years research under the supervision of Prof. Frieder Mugele at Physics of Complex Fluids group in University of Twente, The Netherlands to obtain the degree of doctor in 2011. 